\title{
Critical exponents for semilinear Tricomi-type equations
}

\author{
Dissertation \\ zur Erlangung des mathematisch-naturwissenschaftlichen Doktorgrades \\ „Doctor rerum naturalium“ \\ der Georg-August-Universität Göttingen \\ im Promotionsprogramm PhD School of Mathematical Sciences (SMS) \\ der Georg-August University School of Science (GAUSS)
}

vorgelegt von

Daoyin He

aus Guizhou, China

Göttingen, 2016 
Betreuungsausschuss

Prof. Dr. Ingo WITT (Mathematisches Institut)

Prof. Dr. Dorothea BAHns (Mathematisches Institut)

Mitglieder der Prüfungskommission

Referent: Prof. Dr. Ingo WITT

Korreferentin: Prof. Dr. Dorothea BAHns

Weitere Mitglieder der Prüfungskommission:

Prof. Dr. Folkert MüLLER-Hoissen (Max-Planck-Institut für Dynamik und Selbstorganisation)

Prof. Dr. Russell Luke (Institut für Numerische und Angewandte Mathematik)

Prof. Dr. Thomas Schick (Mathematisches Institut)

Prof. Dr. Viktor Pidstrygach (Mathematisches Institut)

Tag der mündlichen Prüfung: 16 September 2016 


\section{Abstract}

In this thesis, we consider the semilinear Tricomi-type equations. In particular, we work on the global Cauchy problem for the semilinear Tricomi-type equation

$$
\partial_{t}^{2} u-t^{m} \Delta u=|u|^{p}
$$

in $\mathbb{R}_{+} \times \mathbb{R}^{n}$ with suitable initial data $\left(u(0, \cdot), \partial_{t} u(0, \cdot)\right)=\left(u_{0}, u_{1}\right)$, where $n \geq 3$ and $m \in \mathbb{N}$. The main objective of this thesis is to determine the critical exponent $p_{c}=p_{c}(m, n)$, such that if $p>p_{c}$, the global existence of small initial data solution is guaranteed, while, for $1<p \leq p_{c}$ and (non-zero) non-negative initial data, the local solution blows up in finite time.

So far, in the joint work with Ingo Witt and Huicheng Yin, we have found out the precise value of the critical exponent $p_{c}$. As it turns out, the Tricomi-type operators behave much like the wave operator. Therefore, non-weighted or weighted Strichartz estimates can establish what ultimately leads to global existence. For the blowup part, one uses a suitable formula for a modified Bessel function together with the test function method to get the result. 



\section{Acknowledgments}

I would first like to express my sincere gratitude to my advisor Prof. Dr. Ingo WITT for his encouragement, guidance, and inspiration during my graduate studies. I am also greatly indebted to Prof. Dr. Dorothea BAHNS for her advice and assistance in writing this thesis.

My special thanks go to my supervisor at Nanjing University, Prof. Dr. Huicheng YIN for his guidance and help in my Ph.D. study in Göttingen.

I am very thankful to Mathematisches Institut at Georg-AugustUniversität Göttingen, which supported my study during the last four years.

I am glad to acknowledge the following persons for helpful discussions, suggestions, and comments: Bingbing Ding, Zhuoping RuAN, Karen YAGDJIAN, Jens WiRTH.

My appreciation also go to Matthias KRÜGER, Yingbo LıU, Thi Thu Huong Nguyen, Ngoc Thai Nguyen, Nhu Thang Nguyen, Ankel Pohl Robin Spratte, Xiaowen Wu, Jiguang Zheng, for their willingness to share their mathematical knowledge with me.

I thank NGuyen Thi Thu Huong and Du Li for sharing their experience in LTEXwith me.

I wish to thank Yaqing Sun for the continued encouragement. I also thank all of my friends for their help during my stay in Göttingen.

Finally, I would like to thank my parents for their love, understanding, and support. Without them, I would never have been able to continue my education. 



\section{Contents}

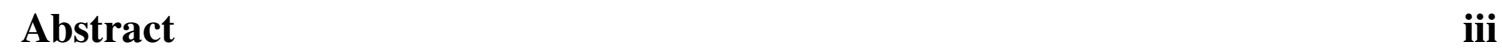

\begin{tabular}{|l|l|}
\hline Acknowledgments & v
\end{tabular}

$\begin{array}{lll}1 & \text { Introduction } & 1\end{array}$

1.1 Tricomi's equation and idea . . . . . . . . . . . . . . . . 1

1.2 The generalized Tricomi equation $\ldots \ldots \ldots$. . . . . . . . 2

1.3 The semilinear problem . . . . . . . . . . . . . . . . 2

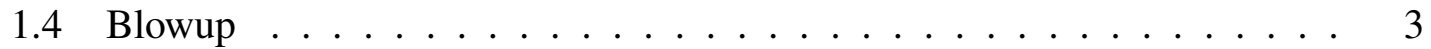

1.5 Global existence . . . . . . . . . . . . . . . . . . 4

1.6 Structure of the thesis $\ldots \ldots \ldots \ldots$. . . . . . . . . . . . . 4

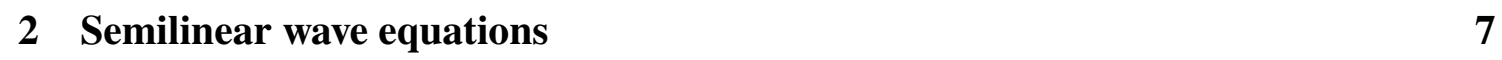

2.1 Historical review . . . . . . . . . . . . . . . . . . . . . 7

2.2 Blowup . . . . . . . . . . . . . . . . . . 8

2.3 Global existence . . . . . . . . . . . . . . . . . . . . . 9

$2.3 .1 \quad$ The case $p \geq p_{0} \quad \ldots \ldots \ldots \ldots$

2.3 .2 The case $p_{c}<p \leq p_{0} \ldots \ldots \ldots \ldots$. . . . . . . . . 10

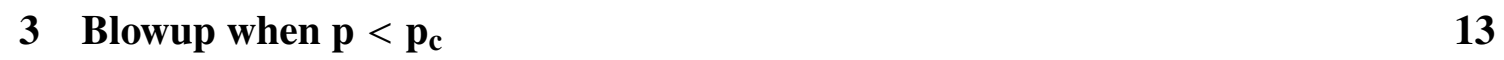

3.1 Derivation of $(2.3) \ldots \ldots \ldots \ldots$

3.2 Derivation of $(2.4) \ldots \ldots \ldots \ldots$

3.3 Proof of Theorem $1.1 \ldots \ldots \ldots \ldots$

4 Global existence for $p \geq p_{0} \quad 19$

4.1 Estimate for linear homogeneous equation . . . . . . . . . . . . . 20

4.2 Estimate for the linear inhomogeneous equation . . . . . . . . . . . . 26

4.3 Proof of Theorem $1.2 \ldots \ldots \ldots \ldots$

4.3.1 The case when $n \geq 3, p$ is small or $n=2 \ldots . \ldots 29$ 
4.3 .2 The case when $n \geq 3, p$ is large $\ldots \ldots . \ldots 31$

5 Weighted Strichartz estimate for the homogeneous equation when $p<p_{0} \quad 35$

5.1 The pointwise estimate . . . . . . . . . . . . . . . 35

$5.1 .1 \quad$ The case ||$x-y|-\phi(t)| \geq C_{0} M \ldots \ldots 36$

5.1.2 The case ||$x-y|-\phi(t)| \leq C_{0} M \ldots \ldots$. . . . . . . . 37

5.2 Weighted Strichartz estimate $\ldots \ldots \ldots$. . . . . . . . . . 38

6 Weighted Strichartz estimate for the inhomogeneous equation. 41

\begin{tabular}{lll}
\hline 7 & $L^{q}$ estimate for $q=q_{0}$ & 47
\end{tabular}

7.1 Proof of Proposition $7.1 \ldots \ldots \ldots$. . . . . . . . . . . . . . . . 48

7.1.1 The case $\operatorname{supp} F(\cdot, y) \subseteq\{s>0: \phi(s)<\phi(1 / 2) / 8\} \ldots 48$

7.1.2 The case $\operatorname{supp} F(\cdot, y) \subseteq\left\{s>0: \phi(s) \geq \frac{1}{8} \phi\left(\frac{1}{2}\right)\right\} \ldots$. . . . . . 50

7.2 Small time . . . . . . . . . . . . . . . . . 53

7.3 Large time . . . . . . . . . . . . . . . . . . . 55

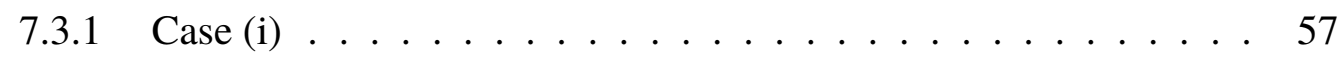

7.3 .2 Case (ii) . . . . . . . . . . . . . . . . . 60

$7.3 .3 \quad$ Case (iii) $\ldots \ldots \ldots \ldots$. . . . . . . . . . . . . 61

\begin{tabular}{|lll}
\hline & $L^{q}$ estimate for $q=2$ & 67
\end{tabular}

$8.1 \quad$ Estimate of $w_{1} \ldots \ldots \ldots \ldots \ldots$. . . . . . . . . . . . . 68

8.2 Estimation of $w_{0} \ldots \ldots \ldots \ldots \ldots$

$8.2 .1 \quad$ The case $\delta \geq 10 \phi(2) \ldots \ldots \ldots \ldots$. . . . . . . . . 69

8.2 .2 The case $\delta_{0} \leq \delta \leq 10 \phi(2) \ldots \ldots \ldots \ldots$

\begin{tabular}{lll}
\hline A & Appendix & 75
\end{tabular}

\begin{tabular}{lr}
\hline Bibliography & 81
\end{tabular} 


\section{Introduction}

The objective of this thesis is to establish global existence and blowup results for the Cauchy problem for the semilinear generalized Tricomi equation. More specifically, for the Cauchy problem

$$
\left\{\begin{array}{l}
\partial_{t}^{2} u-t^{m} \Delta u=|u|^{p}, \\
u(0, x)=u_{0}(x), \quad \partial_{t} u(0, x)=u_{1}(x),
\end{array}\right.
$$

where $t \geq 0, x \in \mathbb{R}^{n}, n \geq 2$, and $m \in \mathbb{N}$, we prove that there exists a critical exponent $p_{c}=p_{c}(m, n)$ such that, for $1<p \leq p_{c}$, local solutions will in general blow up in finite time, whereas, for $p>p_{c}$, global existence of small data solutions hold.

\subsection{Tricomi's equation and idea}

In 1923, Tricomi [34] initiated work on boundary value problems for partial differential equations of mixed type and related equations of changing type. The Tricomi operator is

$$
\mathcal{T}=\partial_{t}^{2}-t \partial_{x}^{2}
$$

in $\mathbb{R}^{2}$. It is elliptic for $t<0$ and hyperbolic for $t>0$. Tricomi considered boundary problems in a mixed-type domain. He transformed the problem into an integral equation and found an explicit formula for the solution.

Frankl [6] drew attention to the fact that the Tricomi problem is closely related to the study of gas flow with nearly sonic speed. More precisely, the Tricomi equation describes the transition from subsonic flow (elliptic region, $t<0$ ) to supersonic flow (hyperbolic region, $t>0$ ), for instance, in a de Laval nozzle, which is one of the most interesting problems in fluid dynamics. 


\subsection{The generalized Tricomi equation}

The generalized Tricomi operator is

$$
\overline{\mathcal{T}}=\partial_{t}^{2}-t^{m} \Delta_{x}
$$

where $t \in \mathbb{R}, x \in \mathbb{R}^{n}, m \in \mathbb{N}$. The well-posedness of the Cauchy problem for (1.3) in the hyperbolic domain $t>0$ has been exhaustively investigated. Moreover, the existence of different fundamental solutions for the Cauchy problem has been established. Barros-Neto and Gelfand [1-3] for the Tricomi equation with $n=1$ and Yagdjian [35] for the generalized Tricomi equation with $n \geq 1$ constructed fundamental solutions of the corresponding operators.

Recently, linear and semilinear generalized Tricomi equations have been in the focus of interest of many authors (see, e.g. Lupo and Payne [17-20]). Ruan, Witt, and Yin [25-27] established the local existence and the local singularity structure of low regularity solutions of the semilinear equation $\partial_{t}^{2} u-t^{m} \Delta u=f(t, x, u)$ in the degenerate hyperbolic regions and the mixed elliptic-hyperbolic regions, respectively, where $f$ is a $C^{1}$ function and has compact support with respect to $x$.

\subsection{The semilinear problem}

We are concerned with the global Cauchy problem

$$
\left\{\begin{array}{l}
\partial_{t}^{2} u-t^{m} \Delta u=F_{p}(u), \\
u(0, x)=\varepsilon u_{0}(x), \quad \partial_{t} u(0, x)=\varepsilon u_{1}(x),
\end{array}\right.
$$

for suitable data $\left(u_{0}, u_{1}\right) \in C_{c}^{\infty}\left(\mathbb{R}^{n}\right)$. Here, the nonlinear term $F_{p}(u)$ is a $C^{1}$ function of $u$. For a given power $p>1, F_{p}(u)$ satisfies

$$
\left|\partial_{u}^{j} F_{p}(u)\right| \leq|u|^{p-j}, \quad j=0,1
$$

Our main goal is to determine the critical exponent $p_{c}=p_{c}(m, n)$ such that, for $1<p \leq p_{c}$, local solutions will in general blow up in finite time, whereas, for $p>p_{c}$, global existence of small data solutions hold.

There are only a few known results concerning this problem. Yadgjian [36] established $L^{p}-L^{q}$ estimates for the solutions $v$ of linear equation $\partial_{t}^{2} v-t^{m} \Delta v=F(t, x)$ and obtained a series of interesting results about the global existence of solutions of problem (1.1) when 
the exponent $p$ is restricted to belong to the range

$$
\left\{\begin{array}{l}
p \leq 1+\frac{2 m}{(m+2) n+2}, \\
((m+2) n-2) p^{2}-((m+2) n+4) p-2 \geq 0 .
\end{array}\right.
$$

In the same paper, Yagdjian applied a method of Sideris [30] and showed blowup of local solutions when $p$ belongs to the interval

$$
1<p<\frac{(k+1) n+1}{(k+1) n-1}
$$

provided the condition

$$
\int_{\mathbb{R}^{n}} u_{1}(x) \mathrm{d} x>0
$$

holds. There was a gap between the global existence interval and the blowup interval, moreover, the critical exponent $p_{c}(m, n)$ was not determined there.

The Cauchy problem for (1.4) with low regularity initial data was considered by Ruan, Witt, and Yin [28].

\subsection{Blowup}

Our first result concerns the blowup of local solutions when $1<p<p_{c}$. To this end, we define, as in [39], for the local solution $u$ the function $G(t)=\int_{\mathbb{R}^{n}} u(t, x) \mathrm{d} x$ and use the multiplier method to show the blowup of $G(t)$ in finite time. If the initial data has compact support, then finite speed of propagation yields blowup of $u$. More specifically, as in [11. 27], investigating the modified Bessel function and choosing a good test function, we derive a Riccati-type ordinary differential inequality for $G(t)$ by a delicate analysis of (1.1). From this and an ODE lemma, the following blowup result can be established assuming positivity of $u_{0}$ and $u_{1}$.

Let $p_{c}$ denote the positive root of

$$
\frac{(m+2) n-2}{2} p^{2}-\frac{(m+2)(n-2)+6}{2} p-(m+2)=0 .
$$

Then we have

Theorem 1.1 (Blowup in finite time). Let $1<p<p_{c}$. Suppose $\left(u_{0}, u_{1}\right) \in H^{1}\left(\mathbb{R}^{n}\right) \times$ $H^{1-\frac{2}{m+2}}\left(\mathbb{R}^{n}\right)$ are nonnegative and positive somewhere, and $\operatorname{supp}\left(u_{0}, u_{1}\right) \subseteq\{x|| x \mid \leq$ $R\}$ for some $R>0$. If problem (1.1) has a solution $u \in C\left([0, T], H^{1}\left(\mathbb{R}^{n}\right)\right) \cap$ $C^{1}\left([0, T], H^{1-\frac{2}{m+2}}\left(\mathbb{R}^{n}\right)\right)$, then $T<\infty$. 


\subsection{Global existence}

Having established Theorem 1.1, a new question arises: Is the positive root $p_{c}(m, n)$ of (1.8) the critical exponent? To answer this, we shall find the range of $p$ where local solutions can be extended for all times. As in the case of wave equation, here the proof is very long and complicated. For technical reason and the reader's convenience, we shall divide proof into two parts, for $p \geq p_{0}$ and $p_{c} \leq p \leq p_{0}$, respectively. The demarcation point is given by

$$
p_{0}=p_{0}(m, n)=\frac{(m+2) n+6}{(m+2) n-2} .
$$

Note that equation (1.1) is conformally invariant for $p=p_{0}$.

For $p \geq p_{0}$, unweighted Strichartz estimates for linear homogeneous equation and inhomogeneous equation yield the global existence theorem:

Theorem 1.2 (Global existence for $\left.\mathbf{p} \geq \mathbf{p}_{\mathbf{0}}\right)$. Let $p_{0} \leq p \leq \frac{(m+2)(n-2)+6}{(m+2)(n-2)-2}$ or else $p>$ $\frac{(m+2)(n-2)+6}{(m+2)(n-2)-2}, p$ is an integer, and $F_{p}(u)$ is replaced with $\pm u^{p}$. Then, there exists a constant $\varepsilon_{0}>0$ such that, for

$$
\left\|u_{0}\right\|_{\dot{H}^{s}}+\left\|u_{1}\right\|_{\dot{H}^{s-\frac{2}{m+2}}} \leq \varepsilon_{0},
$$

problem (1.1) admits a global weak solution $u \in L^{r}\left(\mathbb{R}_{+}^{n+1}\right)$. Here $s=\frac{n}{2}-\frac{4}{(m+2)(p-1)}$ and $r=\frac{((m+2) n+2)(p-1)}{4}$.

Remark 1.3. In [28], it is proved that $u \in C\left([0, \infty), \dot{H}^{s}\left(\mathbb{R}^{n}\right)\right)$.

In case $p_{c} \leq p \leq p_{0}$, the method that leads to the proof of Theorem 1.2 does not work any more. Indeed, we need a refined decay estimates when $p$ is near $p_{c}$. To get this, we follow an idea of Geogiev, Lindblad, and Sogge [7] and define the $L^{p}$ norm with characteristic weight $\left(\frac{4}{(m+2)^{2}} t^{m+2}-|x|^{2}\right)^{\gamma}$ for the generalized Tricomi operator $\partial_{t}^{2}-t^{m} \Delta$. By applying an explicit formula for the solution $v$ of the problem $\partial_{t}^{2} v-t^{m} \Delta v=F(t, x)$, $\left(v(0, x), \partial_{t} v(0, x)\right)=(f(x), g(x))$, we arrived at a class of Fourier integral operators associated with the operator $\partial_{t}^{2}-t^{m} \Delta$. From here, a series of crucial weighted inequalities of $v$ is established by a rather delicate and involved analysis. Notice, however, that we still need to handle some remaining cases to finally get the global existence result. This will be subject of a forthcoming paper.

\subsection{Structure of the thesis}

This thesis is organised as follows. 
Chapter 2 reviews the theory of semilinear wave equations, this work is closely related to this case. We also recall Strauss' conjecture. We then review the history of resolving this conjecture, describe those ideas and useful techniques which motivated our approach.

Chapter 3 contains a proof of the blowup result for $1<p<p_{c}$. We follow an idea of Glassey [9] and Sideris [30], prove blowup by using a lemma on ODEs and by applying some refined techniques for the modified Bessel function as in [11, 27].

Chapter 4 is devoted to the proof of global existence result for $p \geq p_{0}$. Motivated by Lindblad and Sogge [15], where Strichartz estimates were obtained for the linear wave operator, we are required to establish Strichartz estimates for the generalized Tricomi operator $\partial_{t}^{2}-t^{m} \Delta$. In this process, a series of inequalities is derived by applying an explicit formula for solutions of the linear generalized Tricomi equations and by utilizing some basic properties of related Fourier integral operators. Based on the resulting inequalities and the contraction mapping principle, we eventually prove global existence for $p \geq p_{0}$.

Chapter 5 concentrates on the case $p_{c}<p<p_{0}$. In this chapter, we will prove a precise pointwise estimate for the solution of linear homogeneous Tricomi type equation, and then use it to get the weighted Strichartz estimate for the homogeneous equation.

In Chapter 6, we study the weighted Strichartz estimate for linear inhomogeneous equation. This is the most difficult part in the whole thesis. We start with reducing the problem to a estimate inside the characteristic cone. Using interpolation, we then only need to establish this estimate for the two endpoints $p=p_{0}$ and $p=1$.

In Chapter 7, we deal with the case $p=p_{0}$. To this end, we follow an idea of Geogiev, Lindblad, and Sogge [7] and split the solution into pieces supported in different parts. Most cases will be handled in this chapter.

Chapter 8 studies the case $p=1$. The idea of the proof is basically the same as in Chapter 5, but with easier computation. These two endpoints estimates together with complex interpolation yield the weighted Strichartz estimates for all indexes $p$.

The thesis concludes with an appendix, which contains some technical stuff and lemmas from other papers. Some useful notes and comments can also be found there. 



\section{Semilinear wave equations}

In this chapter, we review the theory of the semilinear wave equation

$$
\left\{\begin{array}{l}
\partial_{t}^{2} u-\Delta u=|u|^{p}, \\
u(0, x)=\varepsilon u_{0}(x), \quad \partial_{t} u(0, x)=\varepsilon u_{1}(x)
\end{array}\right.
$$

After stating the problem and listing several important results about this problem, we introduce some basic ideas for the wave equation, then some technical stuff are given for both blowup and global existence. Finally, we show how these knowledge motivates our approach to the problem under study.

\subsection{Historical review}

Here we provide some historical background. John [13] in 1979 showed that for $n=3$ global solutions of (2.1) always exist provided $p>1+\sqrt{2}$ and $\varepsilon>0$ is small. He also showed that this exponent is critical in the sense that no such result can hold for $p<1+\sqrt{2}$. It was shown later by Schaeffer [29] that there in general is also blowup for arbitrarily small data in the case $n=3$ and $p=1+\sqrt{2}$.

The number $1+\sqrt{2}$ first appeared in Strauss' work on scattering of small-amplitude semilinear Schrödinger equations. Since semilinear wave equations and semilinear Schrödinger equations behave similarly in several ways, he made the insightful conjecture [32] that, for $n \geq 2$, global solutions of (2.1) should always exist if $\varepsilon$ is small and $p$ is greater than a critical power $p_{c}$ which is the positive root of the quadratic equation

$$
(n-1) p^{2}-(n+1) p-2=0 .
$$

This conjecture was shortly after verified for $n=2$ by Glassey [8, 9]. John's blow up results were then extended by T. Sideris [30]. He showed that, for all $n \geq 4$, there is in general blowup for arbitrarily small data if $p<p_{c}$. In the other direction, Zhou [38] showed that when $n=4$, there is always global existence for small data solutions if $p>p_{c}$. 
The result has been extended to dimensions $n \leq 8$ by Lindblad and Sogge [16]. Here it was also shown that, under the assumption of spherical symmetry, for arbitrary $n \geq 3$ global solutions of (2.1) exist if $p>p_{c}$ and $\varepsilon$ is small. Later, for $n \geq 4$, Georgiev, Lindblad, and Sogge [7] removed the restriction of symmetry. For the critical case $p=p_{c}$, Schaeffer [29] proved blowup for $n=3$. Finally, Yordanov and Zhang [39] finished the proof by showing blowup for all $n \geq 4$.

\subsection{Blowup}

Suppose we have a local solution $u$ of (2.1) satisfying $\left(u, \partial_{t} u\right) \in C\left([0, T), H^{1}\left(\mathbb{R}^{n}\right)\right) \times$ $C\left([0, T), L^{2}\left(\mathbb{R}^{n}\right)\right)$. We want to show blowup in finite time. The idea is to introduce the function $G(t)=\int_{\mathbb{R}^{n}} u(t, x) \mathrm{d} x$. Since the speed of propagation is finite, once we have proven blowup of $G(t)$, then blowup of $u$ immediately follows for initial data of compact support.

To this end, we need the following lemma which implicitly appeared in [9].

Lemma 2.1. Suppose that $G \in C^{2}([a, b))$ is real valued and, for $a \leq t<b$,

$$
\begin{aligned}
G(t) & \geq C_{0}(R+t)^{\alpha}, \\
G^{\prime \prime}(t) & \geq C_{1}(R+t)^{-q} G(t)^{p},
\end{aligned}
$$

where $C_{0}, C_{1}$, and $R$ are positive constants. Suppose further that $p>1, \alpha \geq 1$, and

$$
(p-1) \alpha \geq q-2 \text {. }
$$

Then $b$ is finite.

With this lemma at hand, the main task is reduced to derive (2.3) and (2.4). To this goal, Sideris [30] for $n \geq 4$ rewrote problem [2.1] as an integral equation and used the Riemann function to establish certain estimates. This computation was long and complicated. His proof was simplified by Rammaha [24] and Zhou [38]. Yordanov and Zhang [39] applied an idea from parabolic equations to derive (2.3) and (2.4) for the critical case, which turned out to be a special multiplier method, and they got the result by quite a short proof.

In this thesis, we use the idea of Yordanov and Zhang. More specifically, they found a special solution $\psi(t, x)$ of the linear homogeneous equation

$$
\partial_{t}^{2} u-\Delta u=0 .
$$

Here $\psi(t, x)=\lambda(t) \phi(x)=e^{-t} \phi(x)$, and $\phi(x)$ is a generalized eigenfunction of Laplacian on $\mathbb{R}^{n}$. Then they used $\psi(t, x)$ as a multiplier and derived a lower bound for $G_{1}(t)=$ 
$\int_{\mathbb{R}^{n}} u(t, x) \psi(t, x) \mathrm{d} x$. This bound played a decisive role in the derivation of (2.3) and (2.4). In our case, since the coefficients of the Tricomi-type equation does not depend on $x$, we can also find a special solution of (2.5) of the form $\lambda(t) \phi(x)$. However, now $\lambda(t)$ is a function related to the modified Bessel equation. We have to use an implicit formula for $\lambda(t)$ to establish (2.3) and (2.4).

\subsection{Global existence}

It has been known for a while that non-trivial space-time estimates for the wave equation lead to improved existence theorems for semilinear wave equations. The pioneering work was Strichartz' [33], who proved a space-time estimate in the case $p=p_{0}=(n+3) /(n-1)$. The essential idea Strichartz estimates is that one gains regularity because the solution of the linear Cauchy problem spreads out in all directions almost as rapidly as in the radial case if the initial data is smooth enough.

\subsubsection{The case $p \geq p_{0}$}

In 1995, for the linear equation

$$
\left\{\begin{array}{l}
\partial_{t}^{2} u-\Delta u=F, \\
u(0, x)=\varepsilon f(x), \quad \partial_{t} u(0, x)=\varepsilon g(x)
\end{array}\right.
$$

Lindblad and Sogge [15] established the estimate

$$
\|u\|_{L_{t}^{s} L_{x}^{q}\left(S_{T}\right)}+\|u\|_{\dot{H}^{\gamma}\left(D_{T}\right)} \leq C\left(\|F\|_{L_{t}^{r} L_{x}^{p}\left(S_{T}\right)}+\|f\|_{\dot{H}^{\gamma}\left(\mathbb{R}^{n}\right)}+\|g\|_{\dot{H}^{\gamma-1}\left(\mathbb{R}^{n}\right)}\right)
$$

provided the gap condition

$$
n\left(\frac{1}{p}-\frac{1}{q}\right)+\frac{1}{r}-\frac{1}{s}=2
$$

and

$$
\frac{1}{p}-\frac{1}{q}=\frac{2}{n+1}, \quad s=\frac{4 q}{(n-1)(q-2)}, \quad \gamma=\frac{n+1}{2}\left(\frac{1}{2}-\frac{1}{q}\right)
$$

for

$$
\begin{cases}\left|\frac{1}{2}-\gamma\right|<\frac{1}{n-1}, & n \geq 3, \\ \left|\frac{1}{2}-\gamma\right|<\frac{1}{4}, & n=2\end{cases}
$$

hold. Here $S_{T}=(0, T) \times \mathbb{R}^{n}$ and $D_{T}=\left\{(T, x) \mid x \in \mathbb{R}^{n}\right\}$. 
Furthermore, if $n \geq 2,2(n+1) /(n-1) \leq q<\infty$, and $\gamma=n / 2-(n+1) / q \geq 1 / 2$, then

$$
\begin{aligned}
& \|u\|_{L^{q}\left(S_{T}\right)}+\left\|\left|D_{x}\right|^{\gamma-1 / 2} u\right\|_{L^{2(n+1) /(n-1)}\left(S_{T}\right)}+\|u\|_{\dot{H}^{\gamma}\left(D_{T}\right)} \\
& \quad \leq C_{q}\left(\left\|\left|D_{x}\right|^{\gamma-1 / 2} F\right\|_{L^{2(n+1) /(n+3)}\left(S_{T}\right)}+\|f\|_{\dot{H}^{\gamma}\left(\mathbb{R}^{n}\right)}+\|g\|_{\dot{H}^{\gamma-1}\left(\mathbb{R}^{n}\right)}\right)
\end{aligned}
$$

The proof uses the theory of Fourier integral operators as the main technical tool. More specifically, in [15] the fundamental solution of (2.6) was used, and the related operator

$$
\left(W^{\alpha} F\right)(t, x)=\iint_{\mathbb{R}^{1+n}} e^{i x \xi+i(t-s)|\xi|} \hat{F}(s, \xi) \frac{\mathrm{d} \xi}{|\xi|^{\alpha}} \mathrm{d} s, \quad \alpha<n,
$$

was studied. This was followed by a use of the Hardy-Littlewood theorem for fractional integrals, the Riesz interpolation theorem and pointwise estimates of the dyadic parts of the kernels

$$
K_{j}^{\alpha}(t, x)=\int_{\left.\mathbb{R}^{n}\right)} e^{i x \xi+i t|\xi|} \beta\left(|\xi| / 2^{j}\right) \frac{\mathrm{d} \xi}{|\xi|^{\alpha}}
$$

for certain $\beta \in C_{c}^{\infty}\left(\mathbb{R}_{+}\right)$. With all these analysis, Lindblad and Sogge managed to get (2.7) and (2.10). Then an iteration proof of this yield:

Theorem 2.2. Let $p \geq(n+3) /(n-1)$, and $p \geq(n+3) /(n-1)$. Set

$$
\gamma=\gamma(p)=\frac{n}{2}-\frac{2}{k-1}
$$

Then, if there exists an $\varepsilon=\varepsilon(n, p)$ such that if,

$$
\|f\|_{\dot{H}^{\gamma}\left(\mathbb{R}^{n}\right)}+\|g\|_{\dot{H}^{\gamma-1}\left(\mathbb{R}^{n}\right)}<\varepsilon
$$

then there is a unique global solution to (2.6) satisfying

$$
\left(u, \partial_{t} u\right) \in C_{b}\left(\mathbb{R} ; \dot{H}^{\gamma}\left(\mathbb{R}^{n}\right) \times \dot{H}^{\gamma-1}\left(\mathbb{R}^{n}\right)\right) \quad \text { and } \quad u \in L^{(p-1)(n+1) / 2}\left(\mathbb{R}^{1+n}\right) .
$$

In this thesis, we use a similar idea, but the computations are much more involved for the fundamental solution of the Tricomi-type operator is more complicated. Especially, we have to carry out a very precise analysis when $t$ is small which is the case when degeneracy happens.

\subsubsection{The case $\mathbf{p}_{\mathrm{c}}<\mathbf{p} \leq \mathbf{p}_{\mathbf{0}}$}

Lindblad and Sogge [16] proved that, under the assumption of spherical symmetry, for arbitrary $n \leq 8$ global solutions of (2.1) exists if $p>p_{c}$ and $\varepsilon$ is small enough. For the general case, John in 1979 proved global existence. The essential part of his argument 
was to establish certain pointwise bounds for the solutions of (2.6) with zero data. More specifically, he proved an inequality which is equivalent to the following:

$$
\left\|t(t-|x|)^{p-2} u\right\|_{L^{\infty}\left(\mathbb{R}_{+}^{1+n}\right)} \leq C_{p}\left\|t^{p}(t-|x|)^{p(p-2)} u\right\|_{L^{\infty}\left(\mathbb{R}_{+}^{1+n}\right)}
$$

if $F(t, x)=0, t-|x| \leq 1$ and $1+\sqrt{2}<p \leq 3$. This fact motivated Georgiev, Lindblad, and Sogge to consider the Strichartz' estimates with characteristic weight $t^{2}-|x|^{2}$ also in higher dimension. They split up $u$ and $F$ into pieces supported at scales of $t$ and $t-|x|$, respectively. After a long and technical proof, which involved harmonic analysis, microlocal analysis, and hyperbolic geometry, they established the following estimate:

Theorem 2.3. Let $n \geq 3$ and assume that $p_{c}<p \leq(n+3) /(n-1)$. Then, if $\varepsilon>0$ is sufficiently small, (1.1) has a unique global solution u such that

$$
(1+|t-| x||)^{\gamma} u \in L^{p+1}\left(\mathbb{R}_{+}^{1+n}\right)
$$

for any $\gamma$ satisfying

$$
\frac{1}{p(p+1)}<\gamma<\frac{(n-1) p-(n+1)}{2(p+1)} .
$$

Based on this, they proved the global existence of weak solutions when $p_{c}<p \leq$ $(n+3) /(n-1)$.

We use a similar idea, but for the Tricomi-type operator here, the pointwise estimate is more difficult and we need to obtain. 



\section{Blowup when $\mathbf{p}<\mathbf{p}_{\mathbf{c}}$}

In this section, we shall prove blowup in finite time for certain local solutions $u$ of $(1.1)$. For a fixed $R>0$, suppose initial data $\left(u_{0}, u_{1}\right) \in C_{c}^{\infty}\left(B_{R}(0)\right)$, where $B_{R}(0)=\{x|| x \mid \leq R\}$ is a ball in $\mathbb{R}^{n}$. By the local existence theory (see [25, 27, 36, 37]), we have, for some $T>0$, a unique solution $u$ satisfying $\left(u, u_{t}\right) \in C\left([0, T), H^{1}\left(\mathbb{R}^{n}\right) \times H^{1-\frac{2}{m+2}}\left(\mathbb{R}^{n}\right)\right)$. Moreover, for $t \in[0, T), \operatorname{supp}\left(u, u_{t}\right)(t, \cdot) \subseteq\{x|| x \mid \leq \phi(t)+R\}$, where $\phi(t)=2 /(m+2) t^{\frac{m+2}{2}}$. As we stated in Section 1.1, we introduce the function $G(t)=\int_{\mathbb{R}^{n}} u(t, x) \mathrm{d} x$. Then the blowup of $u(t, \cdot)$ in $L^{1}\left(\mathbb{R}^{n}\right)$ and hence in $L^{\infty}\left(\mathbb{R}^{n}\right)$ follows from the blowup of $G(t)$, both blowing up at the same time. By some delicate analysis, we obtain a Riccati-type differential inequality for $G(t)$ so that blowup of $G(t)$ can be deduced from Lemma 1.1. Thus our main task is reduced to derive (2.3) and (2.4).

\subsection{Derivation of (2.3)}

Since, for any fixed $t>0$, the support of $u(t, \cdot)$ with respect to the variable $x$ is contained in the ball $B_{R+\phi(t)}(0)$, we have by an integration by parts that

$$
G^{\prime \prime}(t)=\int_{\mathbb{R}^{n}}|u(t, x)|^{p} \mathrm{~d} x \geq \frac{\left|\int_{\mathbb{R}^{n}} u(t, x) \mathrm{d} x\right|^{p}}{\left(\int_{|x| \leq R+\phi(t)} \mathrm{d} x\right)^{p-1}} \geq C(R+t)^{-\frac{m+2}{2} n(p-1)}|G(t)|^{p} .
$$

This shows that $G(t)$ fulfills inequality (2.3) with $q=(m+2) n(p-1) / 2$, once inequality (2.4) has been verified demonstrating that $G$ is positive.

\subsection{Derivation of (2.4)}

To establish (2.4), we introduce the following two functions: the first one is

$$
\varphi(x)=\int_{\mathbb{S}^{n-1}} e^{x \cdot \omega} \mathrm{d} \omega
$$


which was already used in [39], where $\varphi(x)$ is also shown to satisfy

$$
\varphi(x) \sim C_{n}|x|^{-\frac{n-1}{2}} e^{|x|} \text { as }|x| \rightarrow \infty .
$$

The second function is the so-called modified Bessel function of order $v$,

$$
K_{v}(t)=\int_{0}^{\infty} e^{-t \cosh z} \cosh (v z) d z, \quad v \in \mathbb{R},
$$

which is a solution of the equation

$$
\left(t^{2} \frac{\mathrm{d}^{2}}{\mathrm{~d} t^{2}}+t \frac{\mathrm{d}}{\mathrm{d} t}-\left(t^{2}+v^{2}\right)\right) K_{v}(t)=0, \quad t>0 .
$$

From page 24 of [5], we have

$$
K_{v}(t)=\sqrt{\frac{\pi}{2 t}} e^{-t}\left(1+O\left(t^{-1}\right)\right) \quad \text { as } t \rightarrow \infty,
$$

provided that $\operatorname{Re} v>-1 / 2$. Set

$$
\lambda(t)=C_{m} t^{\frac{1}{2}} K_{\frac{1}{m+2}}\left(\frac{2}{m+2} t^{\frac{m+2}{2}}\right), \quad t>0,
$$

where the constant $C_{m}>0$ is chosen so that $\lambda(t)$ satisfies

$$
\left\{\begin{array}{l}
\lambda^{\prime \prime}(t)-t^{m} \lambda(t)=0, \quad t \geq 0 \\
\lambda(0)=1, \quad \lambda(\infty)=0
\end{array}\right.
$$

It follows a list of properties of $\lambda(t)$ (see [11, Lemma 2.1]):

Lemma 3.1. (i) $\lambda(t)$ and $-\lambda^{\prime}(t)$ are both stirctly decreasing. Moreover,

$$
\lim _{t \rightarrow \infty} \lambda(t)=\lim _{t \rightarrow \infty} \lambda^{\prime}(t)=0 .
$$

(ii) There exists a constant $C>1$ such that

$$
\frac{1}{C} \leq \frac{\left|\lambda^{\prime}(t)\right|}{\lambda(t) t^{\frac{m}{2}}} \quad \text { for } t>0 \quad \text { and } \quad \frac{\left|\lambda^{\prime}(t)\right|}{\lambda(t) t^{\frac{m}{2}}} \leq C \quad \text { for } t \geq 1 .
$$

We now introduce the test function $\psi$ with

$$
\psi(t, x)=\lambda(t) \varphi(x)
$$

where the definition of $\varphi$ has been given in 3.2 . Let

$$
G_{1}(t)=\int_{\mathbb{R}^{n}} u(t, x) \psi(t, x) \mathrm{d} x .
$$

Then

$$
G^{\prime \prime}(t)=\int_{\mathbb{R}^{n}}|u(t, x)|^{p} \mathrm{~d} x \geq \frac{\left|G_{1}(t)\right|^{p}}{\left(\int_{|x| \leq M+\phi(t)} \psi(t, x)^{\frac{p}{p-1}} \mathrm{~d} x\right)^{p-1}} .
$$

For the function $G_{1}(t)$, we have: 
Lemma 3.2. Under the assumptions of Theorem 1.1 there exists a $t_{0}>0$ such that

$$
G_{1}(t) \geq C t^{-\frac{m}{2}}, \quad t \geq t_{0} .
$$

Proof. In view of $u \in C\left([0, T), H^{1}\left(\mathbb{R}^{n}\right)\right)$, one has that $G_{1}(t)$ is a continuous function of $t$. Because $u_{0} \geq 0$ and $u_{0} \not \equiv 0$, we have

$$
G_{1}(0)=\int_{\mathbb{R}^{n}} u_{0}(x) \varphi(x) \mathrm{d} x \geq c_{0},
$$

where $c_{0}$ is a positive constant. Hence, there exists a constant $t_{1}>0$ such that, for $0 \leq t \leq t_{1}$,

$$
G_{1}(t) \geq \frac{c_{0}}{2}
$$

Similarly, by Lemma 3.2 (i) and $u_{1} \geq 0$ and $u_{1} \not \equiv 0$, we can also choose a constant $t_{2}>0$ such that, for $0 \leq t \leq t_{2}$,

$$
\int_{\mathbb{R}^{n}} \partial_{t} u(t, x) \psi(t, x) \mathrm{d} x \geq \frac{c_{0}}{2}>0 .
$$

Moreover, by the smoothness of $\lambda(t)$ and $\lambda(0)=1$, we find a $t_{3}>0$ such that

$$
t_{3}^{m / 2} \lambda\left(t_{3}\right) \geq c_{1}
$$

where $c_{1}>0$ is some positive constant. Together with (i) and (ii) of Lemma 3.1, this yields, for $0 \leq t \leq t_{3}$,

$$
-\lambda^{\prime}(t) \geq-\lambda^{\prime}\left(t_{3}\right)=\left|\lambda^{\prime}\left(t_{3}\right)\right| \geq C t_{3}^{m / 2} \lambda\left(t_{3}\right) \geq C c_{1} .
$$

Then, by the assumption that $u_{0} \geq 0$ but $u_{0} \not \equiv 0$, we have that, for $0 \leq t \leq t_{3}$,

$$
\int_{\mathbb{R}^{n}}\left(-\partial_{t} \psi(t, x) u(t, x)\right) \mathrm{d} x \geq \frac{c_{2}}{2}>0,
$$

where $c_{2}$ is a positive constant. Note that

$$
\Delta_{x}\left(\int_{\mathbb{S}^{n-1}} e^{x \cdot \omega} \mathrm{d} \omega\right)=\int_{\mathbb{S}^{n-1}} \sum_{i=1}^{n} \omega_{i}^{2} e^{x \cdot \omega} \mathrm{d} \omega=\int_{\mathbb{S}^{n-1}} e^{x \cdot \omega} \mathrm{d} \omega .
$$

Let $t_{4}=\min \left\{t_{1}, t_{2}, t_{3}\right\}>0$. Then it follows from a direct computation that, for $t>t_{4}$,

$$
\begin{aligned}
\int_{t_{4}}^{t} \int_{\mathbb{R}^{n}}|u|^{p} \psi \mathrm{d} x \mathrm{~d} s & =\int_{t_{4}}^{t} \int_{\mathbb{R}^{n}}\left(\partial_{s}^{2} u-s^{m} \Delta u\right) \psi \mathrm{d} x \mathrm{~d} s \\
& =\left.\int_{\mathbb{R}^{n}}\left(\psi \partial_{s} u-u \partial_{s} \psi\right) \mathrm{d} x\right|_{s=t}-\left.\int_{\mathbb{R}^{n}}\left(\psi \partial_{s} u-u \partial_{s}\right) \mathrm{d} x\right|_{s=t_{4}},
\end{aligned}
$$

which leads to

$$
\left.\int_{\mathbb{R}^{n}}\left(\psi \partial_{s} u-u \partial_{s} \psi\right) \mathrm{d} x\right|_{s=t} \geq\left.\int_{\mathbb{R}^{n}}\left(\psi \partial_{s} u-u \partial_{s} \psi\right) \mathrm{d} x\right|_{s=t_{4}} \geq c \equiv \frac{c_{0}}{2}+\frac{c_{2}}{2} .
$$


This further yields

$$
\begin{aligned}
G_{1}^{\prime}(t)-2 \lambda^{\prime}(t) \int_{\mathbb{R}^{n}} u \varphi \mathrm{d} x & =\frac{\mathrm{d}}{\mathrm{d} t}\left(\int_{\mathbb{R}^{n}} u \psi d x\right)-2 \int_{\mathbb{R}^{n}} u \partial_{t} \psi \mathrm{d} x \\
& =\left.\int_{\mathbb{R}^{n}}\left(\psi \partial_{s} u-u \partial_{s} \psi\right) \mathrm{d} x\right|_{s=t} \geq c .
\end{aligned}
$$

Now assume that there is a constant $t_{5}>t_{4} \operatorname{such} G_{1}\left(t_{5}\right)=0$, but $G_{1}(t)>0$ for $t_{4} \leq t<t_{5}$. Then, for $t_{4} \leq t \leq t_{5}$,

$$
\lambda(t) \int_{\mathbb{R}^{n}} u(t, x) \varphi(x) \mathrm{d} x=\int_{\mathbb{R}^{n}} u(t, x) \psi(t, x) \mathrm{d} x=G_{1}(t) \geq 0 .
$$

Together with Lemma 3.1 (i), this yields that, for $t_{4} \leq t \leq t_{5}$,

$$
\int_{\mathbb{R}^{n}} u(t, x) \varphi(x) \mathrm{d} x \geq 0 .
$$

Furthermore, by Lemma 3.1(ii), we have

$$
-\lambda^{\prime}(t)=\left|\lambda^{\prime}(t)\right| \leq C \lambda(t) t^{\frac{m}{2}} .
$$

Together with (3.12), this yields

$$
G_{1}^{\prime}(t)+C t^{\frac{m}{2}} G_{1}(t) \geq G_{1}^{\prime}(t)-2 \lambda^{\prime}(t) \int_{\mathbb{R}^{n}} u \varphi \mathrm{d} x \geq c .
$$

Without loss of generality, we can assume that $c=1$ in (2.12). Then, by solving (3.13), we get that, for $t_{4} \leq t \leq t_{5}$,

$$
e^{C \phi(t)} G_{1}(t) \geq e^{C \phi\left(t_{4}\right)} G_{1}\left(t_{4}\right)+\frac{t^{-\frac{m}{2}}}{C}\left(e^{C \phi(t)}-e^{C \phi\left(t_{4}\right)}\right) .
$$

Therefore, $G_{1}\left(t_{5}\right)>0$ holds which is a contradiction to $G_{1}\left(t_{5}\right)=0$.

Thus, we have that, for all $t \geq t_{4}$,

$$
G_{1}(t)>0 \text {. }
$$

Using Lemma 3.1 (ii) again and repeating the argument from above, one easily obtains the existence of a uniform positive constant $\tilde{C}$ such that, for $t \geq t_{4}$,

$$
G_{1}(t) \geq \tilde{C} t^{-\frac{m}{2}}
$$

This proves Lemma 3.2 .

The lower bound Lemma 3.2 is the most important part in the proof of blowup. In the case of the wave equation, Yordanov and Zhang proved a similar result, see Lemma 2.2 of [39]. The proof in [39] is simpler and direct. One should also notice that, for the wave equation, the lower bound in Lemma 2.2 of [39] holds for $t \geq t_{0}>0$, where $t_{0}$ can be any positive number. On the contrary, for the Tricomi operator we can only prove the lower bound for $t>t_{4}$, where $t_{4}$ is a certain positive number and we can not make it arbitrarily small. This is due to the degeneracy of Tricomi operator when $t \rightarrow 0$. 


\subsection{Proof of Theorem 1.1}

Relying on Lemma 3.2, we are now able to prove Theorem 1.1 .

Proof of Theorem 1.1. By (3.4) and (3.5), we have that

$$
\lambda(t) \sim t^{-\frac{m}{4}} e^{-\phi(t)} \quad \text { as } t \rightarrow \infty
$$

Next we estimate the denominator $\left(\int_{|x| \leq M+\phi(t)} \psi(t, x)^{\frac{p}{p-1}} \mathrm{~d} x\right)^{p-1}$ in 3.10 . Note that

$$
\left(\int_{|x| \leq M+\phi(t)} \psi(t, x)^{\frac{p}{p-1}} \mathrm{~d} x\right)^{p-1}=\lambda(t)^{p}\left(\int_{|x| \leq M+\phi(t)} \varphi(x)^{\frac{p}{p-1}} \mathrm{~d} x\right)^{p-1}
$$

and

$$
|\varphi(x)| \leq C_{n}(1+|x|)^{-\frac{n-1}{2}} e^{|x|} .
$$

Then

$$
\begin{aligned}
& \int_{|x| \leq M+\phi(t)} \varphi(x)^{\frac{p}{p-1}} \mathrm{~d} x \\
& \quad \leq C \int_{0}^{\frac{M+\phi(t)}{2}}(1+r)^{n-1-\frac{n-1}{2} \cdot \frac{p}{p-1}} e^{\frac{p}{p-1} r} \mathrm{~d} r+C \int_{\frac{M+\phi(t)}{2}}^{M+\phi(t)}(1+r)^{n-1-\frac{n-1}{2} \cdot \frac{p}{p-1}} e^{\frac{p}{p-1} r} \mathrm{~d} r \\
& \quad \leq C e^{\frac{p}{p-1} \cdot \frac{M+\phi(t)}{2}}+(M+\phi(t))^{n-1-\frac{n-1}{2} \cdot \frac{p}{p-1}} e^{\frac{p}{p-1}(M+\phi(t))} \\
& \leq C(M+\phi(t))^{n-1-\frac{n-1}{2} \cdot \frac{p}{p-1}} e^{\frac{p}{p-1}(M+\phi(t))}
\end{aligned}
$$

and

$$
\begin{aligned}
\left(\int_{|x| \leq M+\phi(t)} \psi(t, x)^{\frac{p}{p-1}} \mathrm{~d} x\right)^{p-1} & \leq C t^{-\frac{m}{4} p} e^{-p \phi(t)}(M+\phi(t))^{(n-1)(p-1)-\frac{n-1}{2} p} e^{p(M+\phi(t))} \\
& \leq C t^{-\frac{m}{4} p}(M+\phi(t))^{(n-1)(p-1)-\frac{n-1}{2} p} .
\end{aligned}
$$

Therefore, it follows from (3.10) and (3.15) that, for $t \geq t_{0}$,

$$
G^{\prime \prime}(t) \geq c t^{-\frac{m}{4} p}(M+\phi(t))^{\frac{n-1}{2} p-(n-1)(p-1)} \geq C t^{\frac{p}{2}}(M+\phi(t))^{n-1-\frac{n}{2} p} .
$$

Integrating (3.16) twice gives

$$
G(t) \geq C(M+t)^{\frac{p}{2}+2+\frac{m+2}{2}\left(n-1-\frac{n}{2} p\right)}+C_{1}\left(t-t_{0}\right)+C_{2} .
$$

Note that if

$$
\frac{p}{2}+2+\frac{m+2}{2}\left(n-1-\frac{n}{2} p\right)>1
$$


holds, then one has, for $t \geq t_{0}$,

$$
G(t) \geq C(M+t)^{\frac{p}{2}+2+\frac{m+2}{2}\left(n-1-\frac{n}{2} p\right)} .
$$

This means that condition (2.3) holds with $\alpha=\frac{p}{2}+2+\frac{m+2}{2}\left(n-1-\frac{n p}{2}\right)$.

To conclude the proof of Theorem 1.1, we now apply Lemma 1.1. For $n \geq 3$, one easily checks that all $p<p_{c}$ satisfy (3.17). For $n=2$, 3.17) is equivalent to $(m+1) p<m+4$. On the other hand, if we take

$$
\alpha=\frac{p}{2}+2+\frac{m+2}{2}\left(n-1-\frac{n}{2} p\right), \quad q=\frac{m+2}{2} n(p-1),
$$

then the condition $(p-1) \alpha>q-2$ in Lemma 1.1 becomes

$$
(p-1)\left(\frac{p}{2}+2+\frac{m+2}{2}\left(n-1-\frac{n}{2} p\right)\right)>\frac{m+2}{2} n(p-1)-2,
$$

which is equivalent to

$$
\left((m+2) \frac{n}{2}-1\right) p^{2}+\left((m+2)\left(1-\frac{n}{2}\right)-3\right) p-(m+2)<0 .
$$

By a direct verification, we have that $p_{c}$ satisfies 1.8 and that $p_{c}<p_{0}$ holds. Furthermore, when $n=2$, by a direct computation we have that

$$
p_{c}(m, 2)=\frac{3+\sqrt{4 m^{2}+12 m+7}}{2(m+1)}
$$

and $(m+1) p_{c}(m, 2)<m+4$. We complete the proof of Theorem 1.1 by appealing to Lemma 2.1 with $a=t_{0}$ and $b=t$.

In a forthcoming work, we will also consider the case $p=p_{c}$, where we will need a stronger ODE result than Lemma 2.1. 


\section{Global existence for $p \geq p_{0}$}

In this chapter, we prove the global existence result when $p \geq p_{0}$. The main tool is unweighted Strichartz estimates for both the linear homogeneous equation and the inhomogeneous equation. These estimates not only play an important role in the proof when $p \geq p_{0}$, but they also are basic for deriving the weighted Strichartz estimates when $1<p \leq p_{0}$.

In order to establish global existence, we need to establish unweighted Strichartz estimates for the operator $\partial_{t}^{2}-t^{m} \Delta$. To this end, we study the linear Cauchy problem

$$
\left\{\begin{array}{l}
\partial_{t}^{2} u-t^{m} \Delta u=F(t, x), \quad(t, x) \in \mathbb{R}_{+}^{1+n}, \\
u(0, \cdot)=f(x), \quad \partial_{t} u(0, \cdot)=g(x) .
\end{array}\right.
$$

Note that the solution $u$ of (4.1) can be written as

$$
u(t, x)=v(t, x)+w(t, x)
$$

where $v$ solves the homogeneous problem

$$
\left\{\begin{array}{c}
\partial_{t}^{2} v-t^{m} \Delta v=0, \quad(t, x) \in \mathbb{R}_{+}^{1+n}, \\
v(0, \cdot)=f(x), \quad \partial_{t} v(0, \cdot)=g(x)
\end{array}\right.
$$

and $w$ solves the inhomogeneous problem with zero initial data

$$
\left\{\begin{array}{l}
\partial_{t}^{2} w-t^{m} \Delta w=F(t, x), \quad(t, x) \in \mathbb{R}_{+}^{1+n}, \\
w(0, \cdot)=0, \quad \partial_{t} w(0, \cdot)=0
\end{array}\right.
$$

Let $\dot{H}^{s}\left(\mathbb{R}^{n}\right)$ denote the homogeneous Sobolev space with norm

$$
\|f\|_{\dot{H}^{s}\left(\mathbb{R}^{n}\right)}=\left\|\left|D_{x}\right|^{s} f\right\|_{L^{2}\left(\mathbb{R}^{n}\right)},
$$

where $\left|D_{x}\right|=\sqrt{-\Delta}$.

If $g \equiv 0$ in 4.2 , we intend to establish the Strichartz-type inequality

$$
\|v\|_{L_{t}^{q} L_{x}^{r}} \leq C\|f\|_{\dot{H}_{x}^{s}}
$$


where $q \geq 1$ and $r \geq 1$ are suitable constants related to $s$. By a scaling argument, one obtains that these indices should satisfy

$$
\frac{1}{q}+\frac{m+2}{2} \cdot \frac{n}{r}=\frac{m+2}{2}\left(\frac{n}{2}-s\right) .
$$

For $n \geq 2, n \in \mathbb{N}$, setting $r=q$ and $s=1 /(m+2)$ in (4.4), we find that

$$
q=q_{0} \equiv \frac{2((m+2) n+2)}{(m+2) n-2}>2
$$

By a scaling argument, we see that problem (1.1) is ill-posed for $u_{0} \in \dot{H}^{s}\left(\mathbb{R}^{n}\right)$ with $s<n / 2-4 /((m+2)(p-1))$. Thus we choose $s=n / 2-4 /((m+2)(p-1))$. In this case, $p \geq p_{0}$ implies $s \geq 1 /(m+2)$.

\subsection{Estimate for linear homogeneous equation}

We now prove:

Theorem 4.1. Let $n \geq 2$ and $v$ solve problem (4.2). Further let $1 /(m+2) \leq s<n / 2$. Then

$$
\|v\|_{L^{q}\left(\mathbb{R}_{+}^{1+n}\right)} \leq C\left(\|f\|_{\dot{H}^{s}\left(\mathbb{R}^{n}\right)}+\|g\|_{\dot{H}^{s-\frac{2}{m+2}\left(\mathbb{R}^{n}\right)}}\right),
$$

where $q=\frac{2((m+2) n+2)}{(m+2)(n-2 s)} \geq q_{0}$. The constant $C>0$ depends on $m$, $n$, and $s$.

Proof. It follows from [36] that the solution $v$ of (4.2) can be written as

$$
v(t, x)=V_{1}\left(t, D_{x}\right) f(x)+V_{2}\left(t, D_{x}\right) g(x),
$$

where the operators $V_{j}\left(t, D_{x}\right)(j=1,2)$ have symbols $V_{j}(t, \xi)$ given by

$$
\begin{aligned}
V_{1}(t, \xi)=V_{1}(t,|\xi|)=\frac{\Gamma\left(\frac{m}{m+2}\right)}{\Gamma\left(\frac{m}{2(m+2)}\right)} e^{\frac{z}{2}} H_{+} & \left(\frac{m}{2(m+2)}, \frac{m}{m+2} ; z\right) \\
+ & \frac{\Gamma\left(\frac{m}{m+2}\right)}{\Gamma\left(\frac{m}{2(m+2)}\right)} e^{-\frac{z}{2}} H_{-}\left(\frac{m}{2(m+2)}, \frac{m}{m+2} ; z\right)
\end{aligned}
$$

and

$$
\begin{aligned}
V_{2}(t, \xi)=V_{2}(t,|\xi|)=t \frac{\Gamma\left(\frac{m+4}{m+2}\right)}{\Gamma\left(\frac{m+4}{2(m+2)}\right)} e^{\frac{z}{2}} H_{+}\left(\frac{m+4}{2(m+2)}, \frac{m+4}{m+2} ; z\right) \\
+t \frac{\Gamma\left(\frac{m+4}{m+2}\right)}{\Gamma\left(\frac{m+4}{2(m+2)}\right)} e^{-\frac{z}{2}} H_{-}\left(\frac{m+4}{2(m+2)}, \frac{m+4}{m+2} ; z\right),
\end{aligned}
$$


where $z=2 i \phi(t)|\xi|$. For $\alpha, v \in \mathbb{R}, \omega \in \mathbb{C}$, we have

$$
\begin{aligned}
& H_{+}(\alpha, v ; \omega)=\frac{e^{-i \pi(v-\alpha)}}{e^{i \pi(v-\alpha)}-e^{-i \pi(v-\alpha)}} \frac{1}{\Gamma(v-\alpha)} \omega^{\alpha-v} \int_{\infty}^{(0+)} e^{-\theta} \theta^{v-\alpha-1}\left(1-\frac{\theta}{\omega}\right)^{\alpha-1} \mathrm{~d} \theta \\
& H_{-}(\alpha, v ; \omega)=\frac{1}{e^{i \pi \alpha}-e^{-i \pi \alpha}} \frac{1}{\Gamma(\alpha)} \omega^{-\alpha} \int_{\infty}^{(0+)} e^{-\theta} \theta^{\alpha-1}\left(1+\frac{\theta}{\omega}\right)^{v-\alpha-1} \mathrm{~d} \theta
\end{aligned}
$$

By Section 3 of [36], one has that, for $\phi(t)|\xi| \geq 1$,

$$
\begin{aligned}
& \left|\partial_{\xi}^{\beta} H_{+}(\alpha, \gamma ; 2 i \phi(t)|\xi|)\right| \leq C(\phi(t)|\xi|)^{\alpha-\gamma}\left(1+|\xi|^{2}\right)^{-\frac{|\beta|}{2}} \\
& \left|\partial_{\xi}^{\beta} H_{-}(\alpha, \gamma ; 2 i \phi(t)|\xi|)\right| \leq C(\phi(t)|\xi|)^{-\alpha}\left(1+|\xi|^{2}\right)^{-\frac{|\beta|}{2}}
\end{aligned}
$$

We only estimate $V_{1}$, since estimating $V_{2}$ is similar. Indeed, up to a factor of $t \phi(t)^{-\frac{m+4}{2(m+2)}}=$ $C_{m} \phi(t)^{-\frac{m}{2(m+2)}}$, the powers of $t$ appearing in $V_{1}$ or $V_{2}$ are the same. Choose $\chi \in C^{\infty}\left(\mathbb{R}_{+}\right)$ such that

$$
\chi(s)=\left\{\begin{array}{cc}
1, & s \geq 2 \\
0, & s \leq 1
\end{array}\right.
$$

Then

$$
\begin{aligned}
V_{1}(t,|\xi|) \hat{f}(\xi) & =\chi(\phi(t)|\xi|) V_{1}(t,|\xi|) \hat{f}(\xi)+(1-\chi(\phi(t)|\xi|)) V_{1}(t,|\xi|) \hat{f}(\xi) \\
& \equiv \hat{v}_{1}(t, \xi)+\hat{v}_{2}(t, \xi)
\end{aligned}
$$

Using (4.7), (4.9), and 4.40), we derive that

$$
\begin{aligned}
v_{1}(t, x)=C_{m}\left(\int_{\mathbb{R}^{n}} e^{i(x \cdot \xi+\phi(t)|\xi|)} a_{11}(t, \xi) \hat{f}(\xi) \mathrm{d} \xi\right. & \\
& \left.+\int_{\mathbb{R}^{n}} e^{i(x \cdot \xi-\phi(t)|\xi|)} a_{12}(t, \xi) \hat{f}(\xi) \mathrm{d} \xi\right),
\end{aligned}
$$

where $C_{m}>0$ is a constant only depending on $m$, and, for $l=1,2$,

$$
\left|\partial_{\xi}^{\beta} a_{1 l}(t, \xi)\right| \leq C_{l \beta}|\xi|^{-|\beta|}(\phi(t)|\xi|)^{-\frac{m}{2(m+2)}}
$$

On the other hand, it follows from [4] that

$$
V_{1}(t,|\xi|)=e^{-\frac{z}{2}} \Phi\left(\frac{m}{2(m+2)}, \frac{m}{m+2} ; z\right),
$$

where $\Phi$ is the confluent hypergeometric function which is analytic with respect to the variable $z=2 i \phi(t)|\xi|$. Then

$$
\left|\partial_{\xi}\left((1-\chi(\phi(t)|\xi|)) V_{1}(t,|\xi|)\right)\right| \leq C(1+\phi(t)|\xi|)^{-\frac{m}{2(m+2)}}|\xi|^{-1}
$$


Similarly, one has

$$
\left|\partial_{\xi}^{\beta}\left((1-\chi(\phi(t)|\xi|)) V_{1}(t,|\xi|)\right)\right| \leq C(1+\phi(t)|\xi|)^{-\frac{m}{2(m+2)}}|\xi|^{-|\beta|}
$$

Thus, we arrive at

$$
v_{2}(t, x)=C_{m}\left(\int_{\mathbb{R}^{n}} e^{i(x \cdot \xi+\phi(t)|\xi|)} a_{21}(t, \xi) \hat{f}(\xi) \mathrm{d} \xi+\int_{\mathbb{R}^{n}} e^{i(x \cdot \xi-\phi(t)|\xi|)} a_{22}(t, \xi) \hat{f}(\xi) \mathrm{d} \xi\right),
$$

where, for $l=1,2$,

$$
\left|\partial_{\xi}^{\beta} a_{2 l}(t, \xi)\right| \leq C_{l \beta}(1+\phi(t)|\xi|)^{-\frac{m}{2(m+2)}}|\xi|^{-|\beta|}
$$

Substituting (4.13) and 4.15) into 4.12) yields

$$
\begin{aligned}
V_{1}\left(t, D_{x}\right) f(x)=C_{m} & \left(\int_{\mathbb{R}^{n}} e^{i(x \cdot \xi+\phi(t)|\xi|)} a_{1}(t, \xi) \hat{f}(\xi) \mathrm{d} \xi\right. \\
& \left.+\int_{\mathbb{R}^{n}} e^{i(x \cdot \xi-\phi(t)|\xi|)} a_{2}(t, \xi) \hat{f}(\xi) \mathrm{d} \xi\right),
\end{aligned}
$$

where the $a_{l}(l=1,2)$ satisfy

$$
\left|\partial_{\xi}^{\beta} a_{l}(t, \xi)\right| \leq C_{l \beta}(1+\phi(t)|\xi|)^{-\frac{m}{2(m+2)}}|\xi|^{-|\beta|}
$$

We only treat the integral $\int_{\mathbb{R}^{n}} e^{i(x \cdot \xi+\phi(t)|\xi|)} a_{1}(t, \xi) \hat{f}(\xi) \mathrm{d} \xi$, since the treatment of the integral $\int_{\mathbb{R}^{n}} e^{i(x \cdot \xi-\phi(t)|\xi|)} a_{2}(t, \xi) \hat{f}(\xi) \mathrm{d} \xi$ is similar. Denote

$$
(A f)(t, x)=\int_{\mathbb{R}^{n}} e^{i(x \cdot \xi+\phi(t)|\xi|)} a_{1}(t, \xi) \hat{f}(\xi) \mathrm{d} \xi
$$

We will show that

$$
\|(A f)(t, x)\|_{L^{q}\left(\mathbb{R}_{+}^{n+1}\right)} \leq C\|f\|_{\dot{H}^{s}\left(\mathbb{R}^{n}\right)} .
$$

Note that if we set

$$
\tilde{a}(t, \xi)=\frac{a_{1}(t, \xi)}{|\xi|^{s}}, \quad \hat{h}(\xi)=|\xi|^{s} \hat{f}(\xi)
$$

then 4.18 is equivalent to

$$
\left\|\int_{\mathbb{R}^{n}} e^{i(x \cdot \xi+\phi(t)|\xi|)} \tilde{a}(t, \xi) \hat{h}(\xi) \mathrm{d} \xi\right\|_{L^{q}\left(\mathbb{R}_{+}^{n+1}\right)} \leq C\|h\|_{L^{2}\left(\mathbb{R}^{n}\right)}
$$

We denote the integral operator in the left-hand side of (4.19) still by $A$. In order to prove 4.19 it suffices to establish its dual version

$$
\left\|A^{*} G\right\|_{L^{2}\left(\mathbb{R}^{n}\right)} \leq C\|G\|_{L^{q^{\prime}}\left(\mathbb{R}_{+}^{1+n}\right)},
$$


where

$$
\left(A^{*} G\right)(y)=\int_{\mathbb{R}^{n}} \int_{\mathbb{R}_{+}^{n+1}} e^{i(y-x) \cdot \xi-\phi(t)|\xi|)} \overline{\tilde{a}(t, \xi)} G(t, x) \mathrm{d} t \mathrm{~d} x \mathrm{~d} \xi
$$

is the adjoint operator of $A, \frac{1}{q^{\prime}}+\frac{1}{q}=1$, and $1 \leq q^{\prime} \leq q_{0}^{\prime}=\frac{2((m+2) n+2)}{(m+2) n+6}$ (note that $\frac{1}{q_{0}^{\prime}}+\frac{1}{q_{0}}=1$ ). In view of

$$
\int_{\mathbb{R}^{n}}\left|\left(A^{*} G\right)(y)\right|^{2} \mathrm{~d} y=\int_{\mathbb{R}_{+}^{1+n}}\left(A A^{*} G\right)(t, x) \overline{G(t, x)} \mathrm{d} t \mathrm{~d} x \leq\left\|A A^{*} G\right\|_{L^{q}\left(\mathbb{R}_{+}^{1+n}\right)}\|G\|_{L^{p}\left(\mathbb{R}_{+}^{1+n}\right)},
$$

one derives that 4.20 holds if

$$
\left\|A A^{*} G\right\|_{L^{q}\left(\mathbb{R}_{+}^{1+n}\right)} \leq C\|G\|_{L^{q^{\prime}\left(\mathbb{R}_{+}^{1+n}\right)}}, \quad 1 \leq q^{\prime} \leq q_{0}^{\prime} .
$$

One can write

$$
\left(A A^{*} G\right)(t, x)=\int_{\mathbb{R}_{+}^{1+n}} \int_{\mathbb{R}^{n}} e^{i((\phi(t)-\phi(\tau))|\xi|+(x-y) \cdot \xi)} \tilde{a}(t, \xi) \overline{\tilde{a}(\tau, \xi)} G(\tau, y) \mathrm{d} \xi \mathrm{d} \tau \mathrm{d} y .
$$

To proceed further we need the following lemma from [15]:

Lemma 4.2. Let $\beta \in C_{0}^{\infty}((1 / 2,2))$ and $\sum_{j=-\infty}^{\infty} \beta\left(2^{-j} \tau\right) \equiv 1$ for $\tau>0$. Define the Littlewood-Paley operators as

$$
G_{j}(t, x)=(2 \pi)^{-n} \int_{\mathbb{R}^{n}} e^{i x \cdot \xi} \beta\left(2^{-j}|\xi|\right) \hat{G}(t, \xi) \mathrm{d} \xi, \quad j \in \mathbb{Z} .
$$

Then

$$
\|G\|_{L_{t}^{s} L_{x}^{q}} \leq C\left(\sum_{j=-\infty}^{\infty}\left\|G_{j}\right\|_{L_{t}^{s} L_{x}^{q}}^{2}\right)^{1 / 2}, \quad 2 \leq q<\infty, 2 \leq s \leq \infty
$$

and

$$
\left(\sum_{j=-\infty}^{\infty}\left\|G_{j}\right\|_{L_{t}^{r} L_{x}^{p}}^{2}\right)^{1 / 2} \leq C\|G\|_{L_{t}^{r} L_{x}^{p}}, \quad 1<p \leq 2,1 \leq r \leq 2
$$

If we choose a function $\beta \in C_{0}^{\infty}((1 / 2,2))$ as in Lemma 4.2 and set $a_{\lambda}(t, \tau, \xi)=$ $\beta(|\xi| / \lambda) \tilde{a}(t, \xi) \overline{\tilde{a}(\tau, \xi)}$ for $\lambda>0$, then we obtain a dyadic decomposition of the operator $A A^{*}$ by

$$
\left(A A^{*}\right)_{\lambda} G=\int_{\mathbb{R}_{+}^{n+1}} \int_{\mathbb{R}^{n}} e^{i((\phi(t)-\phi(\tau))|\xi|+(x-y) \cdot \xi)} a_{\lambda}(t, \tau, \xi) G(\tau, y) \mathrm{d} \xi \mathrm{d} \tau \mathrm{d} y .
$$

In order to prove (4.22), we only need to prove

$$
\left\|\left(A A^{*}\right)_{\lambda} G\right\|_{L^{q}\left(\mathbb{R}_{+}^{n+1}\right)} \leq C\|G\|_{L^{q^{\prime}\left(\mathbb{R}_{+}^{n+1}\right)}}, \quad 1 \leq q^{\prime} \leq q_{0}^{\prime},
$$


where the constant $C>0$ is independent of $\lambda>0$. Indeed, if (4.25) holds, then it follows from Lemma 4.2 and $q \leq q_{0}^{\prime}=\frac{2((m+2) n+2)}{(m+2) n+6}<2$ that

$$
\begin{aligned}
\left\|A A^{*} G\right\|_{L^{q}}^{2} & \leq C \sum_{j \in \mathbb{Z}}\left\|\left(A A^{*}\right)_{2^{j}} G\right\|_{L^{q}}^{2} \leq C \sum_{j \in \mathbb{Z}} \sum_{k:|j-k| \leq C_{0}}\left\|\left(A A^{*}\right)_{2^{j}} G_{k}\right\|_{L^{q}}^{2} \\
& \leq C \sum_{j \in \mathbb{Z} k:|j-k| \leq C_{0}} \sum_{L_{k}}\left\|G_{L^{p}}^{2} \leq C\right\| G \|_{L^{p}\left(\mathbb{R}_{+}^{n+1}\right)}^{2}
\end{aligned}
$$

where $\hat{G}_{k}(\tau, \xi)=\beta\left(2^{-k}|\xi|\right) \hat{G}(\tau, \xi)$.

Next we prove 4.25 . We will use interpolation between the two cases $q=\infty$ and $q=q_{0}$.

For $q=\infty$, a direct analysis shows that

$$
\left|a_{\lambda}(t, \tau, \xi)\right| \leq|\xi|^{-2 s}
$$

and

$$
\begin{aligned}
\left\|\left(A A^{*}\right)_{\lambda} G\right\|_{L^{\infty}\left(\mathbb{R}_{+}^{1+n}\right)} & \leq \int_{\mathbb{R}_{+}^{1+n}}\left|\int_{\mathbb{R}^{n}} e^{i[(\phi(t)-\phi(\tau))|\xi|+(x-y) \cdot \xi]} a_{\lambda}(t, \tau, \xi) \mathrm{d} \xi\right| G(\tau, y) \mid \mathrm{d} y \mathrm{~d} \tau \\
& \leq\left.\int_{\mathbb{R}_{+}^{1+n}}\left|\int_{\mathbb{R}^{n}} \beta\left(\frac{|\xi|}{\lambda}\right)\right| \xi\right|^{-2 s} \mathrm{~d} \xi|G(\tau, y)| \mathrm{d} y \mathrm{~d} \tau \\
& \leq C \lambda^{n-2 s}\|G\|_{L^{1}\left(\mathbb{R}_{+}^{n+1}\right)}
\end{aligned}
$$

Next we prove the endpoint case $q=q_{0}$ in 4.25 . Namely, we shall show that

$$
\left\|\left(A A^{*}\right)_{\lambda} G\right\|_{L^{q}\left(\mathbb{R}_{+}^{1+n}\right)} \leq C \lambda^{\frac{2}{m+2}-2 s}\|G\|_{L^{p_{0}\left(\mathbb{R}_{+}^{1+n}\right)}} .
$$

Note that, for any $t, \tau \in \mathbb{R}_{+}$and $\bar{t}=\max \{t, \tau\}$, one has that

$$
\left|\partial_{\xi}^{\beta}\left(\bar{t}^{\frac{m}{(m+2) n+2}} a_{\lambda}(t, \tau, \xi)\right)\right| \leq C|\xi|^{-2 s-\frac{2 m}{(m+2)((m+2) n+2)}-|\beta|} .
$$

Indeed, without loss of generality, one can assume that $t \geq \tau$. Then it follows from 4.16 and a direct computation that

$$
\begin{aligned}
\left|\partial_{\xi}^{\beta}\left(\bar{t}^{\frac{m}{(m+2) n+2}} a_{\lambda}(t, \tau, \xi)\right)\right| & \leq C t^{\frac{m}{(m+2) n+2}}(1+\phi(t)|\xi|)^{-\frac{m}{2(m+2)}}(1+\phi(\tau)|\xi|)^{-\frac{m}{2(m+2)}}|\xi|^{-|\beta|-2 s} \\
& \leq C \phi(t)^{\frac{2 m}{(m+2)((m+2) n+2)}}(\phi(t)|\xi|)^{-\frac{2 m}{(m+2)((m+2) n+2)}}|\xi|^{-|\beta|-2 s} \\
& \leq C|\xi|^{-2 s-\frac{2 m}{(m+2)((m+2) n+2)}-|\beta|} .
\end{aligned}
$$

Set

$$
b(t, \tau, \xi)=\lambda^{2 s+\frac{2 m}{(m+2)((m+2) n+2)}} \bar{t}^{\frac{m}{(m+2) n+2}} a_{\lambda}(t, \tau, \xi) .
$$

Then

$$
\left|\partial_{\xi}^{\beta} b(t, \tau, \xi)\right| \leq|\xi|^{-|\beta|}
$$


and we can write

$$
\begin{aligned}
\left(A A^{*}\right)_{\lambda} G=\int_{\mathbb{R}_{+}^{n+1}} \int_{\mathbb{R}^{n}} e^{i((\phi(t)-\phi(\tau))|\xi|+(x-y) \cdot \xi)} \bar{t}^{-\frac{m}{(m+2) n+2}} \lambda^{-2 s-\frac{2 m}{(m+2)((m+2) n+2)}} & \\
& \times b(t, \tau, \xi) G(\tau, y) \mathrm{d} \xi \mathrm{d} y \mathrm{~d} \tau .
\end{aligned}
$$

Introduce the operator

$$
T_{t, \tau} f(x)=\iint e^{i((\phi(t)-\phi(\tau))|\xi|+(x-y) \cdot \xi)} \bar{t}^{-\frac{m}{(m+2) n+2}} b(t, \tau, \xi) f(y) \mathrm{d} \xi \mathrm{d} y .
$$

Then, by $\max \{t, \tau\} \geq|t-\tau|$, we have that

$$
\left\|T_{t, \tau} f\right\|_{L^{2}\left(\mathbb{R}^{n}\right)} \leq C|t-\tau|^{-\frac{m}{(m+2) n+2}}\|f\|_{L^{2}\left(\mathbb{R}^{n}\right)}
$$

On the other hand, it follows from the method of stationary phase that

$$
\begin{aligned}
\left\|T_{t, \tau} f\right\|_{L^{\infty}\left(\mathbb{R}^{n}\right)} & \leq C \lambda^{\frac{n+1}{2}} \bar{t}^{-\frac{m}{(m+2) n+2}}|\phi(t)-\phi(\tau)|^{-\frac{n-1}{2}}\|f\|_{L^{1}\left(\mathbb{R}^{n}\right)} \\
& \leq C \lambda^{\frac{n+1}{2}}|t-\tau|^{-\frac{m}{(m+2) n+2}}|t-\tau|^{-\frac{n-1}{2} \cdot \frac{m+2}{2}}\|f\|_{L^{1}\left(\mathbb{R}^{n}\right)} .
\end{aligned}
$$

Together with (4.29), this yields

$$
\left\|T_{t, \tau} f\right\|_{L^{q_{0}\left(\mathbb{R}^{n}\right)}} \leq C \lambda^{\frac{2(n+1)}{(m+2) n+2}}|t-\tau|^{-\frac{(m+2) n-2}{(m+2) n+2}}\|f\|_{L^{q_{0}^{\prime}\left(\mathbb{R}^{n}\right)}} .
$$

Because of $1-\left(\frac{1}{q_{0}^{\prime}}-\frac{1}{q_{0}}\right)=\frac{(m+2) n-2}{(m+2) n+2}$, it follows from the Hardy-Littlewood-Sobolev inequality that

$$
\begin{aligned}
& \left\|\left(A A^{*}\right)_{\lambda} G\right\|_{L^{q_{0}\left(\mathbb{R}_{+}^{1+n}\right)}}=\left\|\int_{0}^{\infty} T_{t, \tau} G \mathrm{~d} \tau\right\|_{L^{q_{0}\left(\mathbb{R}_{+}^{1+n}\right)}} \\
& \quad \leq C \lambda^{-2 s-\frac{2 m}{(m+2)((m+2) n+2)}} \lambda^{\frac{2(n+1)}{(m+2) n+2}}\left\|\int_{\mathbb{R}}|t-\tau|^{-\frac{(m+2) n-2}{(m+2) n+2}}\right\| G(\tau, \cdot)\left\|_{L^{q^{\prime}\left(\mathbb{R}^{n}\right)}} \mathrm{d} \tau\right\|_{L^{q_{0}^{\prime}(\mathbb{R})}} \\
& \quad \leq C \lambda^{-2 s+\frac{2}{m+2}}\|G\|_{L^{q_{0}^{\prime}\left(\mathbb{R}_{+}^{1+n}\right)}} .
\end{aligned}
$$

By interpolation between (4.26) and (4.32), we have that, for $1 \leq p \leq p_{0}$,

$$
\left\|\left(A A^{*}\right)_{\lambda} G\right\|_{L^{q}\left(\mathbb{R}_{+}^{n+1}\right)} \leq C \lambda^{-2 s+2\left(\frac{n}{2}-\frac{(m+2) n+2}{(m+2) q}\right)}\|G\|_{L^{p}\left(\mathbb{R}_{+}^{n+1}\right)} .
$$

In particular, choosing $s=\frac{n}{2}-\frac{(m+2) n+2}{(m+2) q}$ yields estimate (4.18) for $v_{1}(t, x)$. The same estimate for $v_{2}(t, x)$ is analogously obtained.

The proof of Theorem 4.1 is complete. 


\subsection{Estimate for the linear inhomogeneous equation}

Next we treat the inhomogeneous problem (4.3). Based on Theorem 4.1 and Lemma 4.2. we establish the following estimate:

Theorem 4.3. Let $n \geq 2$ and $w$ solve (4.3). Then

$$
\|w\|_{L^{q}\left(\mathbb{R}_{+}^{1+n}\right)} \leq C\left\|\left|D_{x}\right|^{\gamma-\frac{1}{m+2}} F\right\|_{L^{\left.q_{\left(\mathbb{R}_{+}\right.}^{1+n}\right)}}
$$

where $\gamma=\frac{n}{2}-\frac{(m+2) n+2}{q(m+2)}, q_{0} \leq q<\infty$, and the constant $C>0$ depends on $m, n$ and $q$.

Proof. It follows from problem 4.3) that

$$
w(t, x)=\int_{0}^{t}\left(V_{2}\left(t, D_{x}\right) V_{1}\left(\tau, D_{x}\right)-V_{1}\left(t, D_{x}\right) V_{2}\left(\tau, D_{x}\right)\right) F(\tau, x) \mathrm{d} \tau .
$$

To estimate $w(t, x)$, it suffices to treat the term $\int_{0}^{t} V_{2}\left(t, D_{x}\right) V_{1}\left(\tau, D_{x}\right) F(\tau, x) \mathrm{d} \tau$, since the treatment on the term $\int_{0}^{t} V_{1}\left(t, D_{x}\right) V_{2}\left(\tau, D_{x}\right) F(\tau, x) \mathrm{d} \tau$ is completely analogous. Choose a cut-off function $\chi$ as in 4.11. Set

$$
\begin{aligned}
& w_{1}(t, x)=\int_{0}^{t} \chi\left(\phi(t) D_{x}\right) \chi\left(\phi(\tau) D_{x}\right) V_{2}\left(t, D_{x}\right) V_{1}\left(\tau, D_{x}\right) F(\tau, x) \mathrm{d} \tau, \\
& w_{2}(t, x)=\int_{0}^{t} \chi\left(\phi(t) D_{x}\right)\left(1-\chi\left(\phi(\tau) D_{x}\right)\right) V_{2}\left(t, D_{x}\right) V_{1}\left(\tau, D_{x}\right) F(\tau, x) \mathrm{d} \tau, \\
& w_{3}(t, x)=\int_{0}^{t}\left(1-\chi\left(\phi(t) D_{x}\right)\right) \chi\left(\phi(\tau) D_{x}\right) V_{2}\left(t, D_{x}\right) V_{1}\left(\tau, D_{x}\right) F(\tau, x) \mathrm{d} \tau, \\
& w_{4}(t, x)=\int_{0}^{t}\left(1-\chi\left(\phi(t) D_{x}\right)\right)\left(1-\chi\left(\phi(\tau) D_{x}\right)\right) V_{2}\left(t, D_{x}\right) V_{1}\left(\tau, D_{x}\right) F(\tau, x) \mathrm{d} \tau .
\end{aligned}
$$

Together with 4.7 -4.40, as in the proof of Theorem 4.1, we can write $\sum_{j=1}^{4} w_{j}$ as

$$
\sum_{j=1}^{4} w_{j}=(A F)(t, x) \equiv \int_{0}^{t} \int_{\mathbb{R}^{n}} e^{i(x \cdot \xi+(\phi(t)-\phi(\tau))|\xi|)} a(t, \tau, \xi) \hat{F}(\tau, \xi) \mathrm{d} \xi \mathrm{d} \tau
$$

where $a(t, \tau, \xi)$ satisfies

$$
\left|\partial_{\xi}^{\beta} a(t, \xi)\right| \leq C(1+\phi(t)|\xi|)^{-\frac{m}{2(m+2)}}(1+\phi(\tau)|\xi|)^{-\frac{m}{2(m+2)}}|\xi|^{-\frac{2}{m+2}-|\beta|} .
$$

To treat $(A F)(t, x)$ conveniently, we introduce the more general operator

$$
\left(A^{\alpha} F\right)(t, x)=\int_{0}^{t} \int_{\mathbb{R}^{n}} e^{i(x \cdot \xi+(\phi(t)-\phi(\tau))|\xi|)} a(t, \tau, \xi) \hat{F}(\tau, \xi) \frac{\mathrm{d} \xi}{|\xi|^{\alpha}} \mathrm{d} \tau,
$$

where $0 \leq \alpha<n / 2$ is a parameter. 
As in the proof of Theorem 4.1, we shall use the Littlewood-Paley argument with a bump function $\beta$ as in Lemma 4.2. Define the operator

$$
A_{j}^{\alpha} F(t, x)=\int_{0}^{t} \int_{\mathbb{R}^{n}} e^{i(x \cdot \xi+(\phi(t)-\phi(\tau))|\xi|)} \beta\left(\frac{|\xi|}{2^{j}}\right) a(t, \tau, \xi) \hat{F}(\tau, \xi) \frac{\mathrm{d} \xi}{|\xi|^{\alpha}} \mathrm{d} \tau .
$$

Note that, for $\gamma=\frac{n}{2}-\frac{(m+2) n+2}{q(m+2)}, q_{0} \leq q<\infty$, our aim is to establish the inequality

$$
\|w\|_{L^{q}\left(\mathbb{R}_{+}^{1+n}\right)} \leq C\left\|\left|D_{x}\right|^{\gamma-\frac{1}{m+2}} F\right\|_{L^{q_{0}^{\prime}\left(\mathbb{R}_{+}^{1+n}\right)}}
$$

which is equivalent to proving that

$$
\left\|\left|D_{x}\right|^{-\gamma+\frac{1}{m+2}} w\right\|_{L^{q}\left(\mathbb{R}_{+}^{1+n}\right)} \leq C\|F\|_{L^{q_{0}^{\prime}\left(\mathbb{R}_{+}^{1+n}\right)}} .
$$

In terms of the operator $A^{\alpha}$ in 4.36 with $\alpha=\gamma-\frac{1}{m+2}$, it suffices to establish

$$
\left\|A^{\alpha} F\right\|_{L^{q}\left(\mathbb{R}_{+}^{1+n}\right)} \leq C\|F\|_{L^{q^{\prime}\left(\mathbb{R}_{+}^{1+n}\right)}}
$$

in order to complete the proof of 4.33.

Note that $p_{0}<2<q<\infty$. It follows from Lemma 4.2 that, in order to derive 4.38) we only need to prove

$$
\left\|A_{j}^{\alpha} F\right\|_{L^{q}\left(\mathbb{R}_{+}^{1+n}\right)} \leq C\|F\|_{L^{q_{(}^{\prime}\left(\mathbb{R}_{+}^{1+n}\right)}} .
$$

By interpolation, it suffices to prove that 4.39) holds for the special cases $q=q_{0}$ and $q=\infty$. Denote the corresponding indices $\alpha$ by $\alpha_{0}$ and $\alpha_{1}$. A direct computation yields $\alpha_{0}=\frac{n}{2}-\frac{(m+2) n+2}{q_{0}(m+2)}-\frac{1}{m+2}=0$ and $\alpha_{1}=\frac{n}{2}-\frac{1}{m+2}$. We now treat $A_{j}^{\alpha_{0}}=A_{j}^{0}$. Let

$$
T_{j}^{0} G(t, \tau, x)=\int_{\mathbb{R}^{n}} e^{i(x \cdot \xi+(\phi(t)-\phi(\tau))|\xi|)} \beta\left(\frac{|\xi|}{2^{j}}\right) a(t, \tau, \xi) \hat{G}(\tau, \xi) \mathrm{d} \xi .
$$

We can repeat the derivation of (4.31) to get

$$
\left\|T_{j}^{0} G(t, \tau, \cdot)\right\|_{L^{q_{0}\left(\mathbb{R}^{n}\right)}} \leq C|t-\tau|^{-\frac{(m+2) n-2}{(m+2) n+2}}\|G(\tau, \cdot)\|_{L^{q_{0}^{\prime}\left(\mathbb{R}^{n}\right)}} .
$$

Note that $A_{j}^{0} G(t, x)=\int_{0}^{t} T_{j}^{0} G(t, \tau, x) \mathrm{d} \tau$. Then, by 4.41) and the Hardy-LittlewoodSobolev inequality, we get

$$
\left\|\int_{\mathbb{R}}\right\| T_{j}^{0} G(t, \tau, x)\left\|_{L_{x}^{q_{0}} \mathrm{~d} \tau}\right\|_{L_{t}^{q_{0}}} \leq C\|G\|_{L^{q_{0}^{\prime}\left(\mathbb{R}^{n}\right)}} .
$$

With

$$
K(t, \tau)= \begin{cases}|t-\tau|^{-\frac{(m+2) n-2}{(m+2) n+2}}, & \tau \geq 0 \\ 0, & \tau<0\end{cases}
$$

it follows from the following lemma with $q=q_{0}$ that 4.39 ) has been obtained. (See Theorem 1.2 of [22] for proof.) 
Lemma 4.4. Let $1 \leq p<q \leq \infty$. Let $T: L^{p}(\mathbb{R}) \rightarrow L^{q}(\mathbb{R})$ be a bounded linear operator which is defined by

$$
T f(x)=\int_{\mathbb{R}} K(x, y) f(y) \mathrm{d} y,
$$

for a locally integrable K. Define

$$
\tilde{T} f(x)=\int_{-\infty}^{x} K(x, y) f(y) \mathrm{d} y .
$$

Then

$$
\|\tilde{T} f\|_{L^{q}} \leq C_{p, q}\|T\|_{L^{p} \rightarrow L^{q}}\|f\|_{L^{p}} .
$$

Next we prove 4.39) for $q=\infty$. In this case, the kernel of $A_{j}^{\alpha_{1}}$ can be written as

$$
K_{j}^{\alpha_{1}}(t, x ; \tau, y)=\int_{\mathbb{R}^{n}} \beta\left(\frac{|\xi|}{2^{j}}\right) e^{i((x-y) \cdot \xi+(\phi(t)-\phi(\tau))|\xi|)} a(t, \tau, \xi) \frac{\mathrm{d} \xi}{|\xi|^{\alpha_{1}}} .
$$

We now assert

$$
\sup _{t, x} \int_{\mathbb{R}_{+}^{n+1}}\left|K_{j}^{\alpha_{1}}(t, x ; \tau, y)\right|^{q_{0}} \mathrm{~d} \tau \mathrm{d} y<\infty .
$$

Obviously, if (4.42) is true, then a direct application of Hölder's inequality yields (4.39) for $q=\infty$.

Next we turn to the proof of (4.42). By [31, Lemma 7.2.4], we have

$$
\begin{aligned}
& \left|K_{j}^{\alpha_{1}}(t, x ; \tau, y)\right| \\
& \leq C_{N, n, \alpha_{1}} \lambda^{\frac{n+1}{2}-\overline{\alpha_{1}}}\left(|\phi(t)-\phi(\tau)|+\lambda^{-1}\right)^{-\frac{n-1}{2}}(1+\lambda|| x-y|-| \phi(t)-\phi(\tau)||)^{-N},
\end{aligned}
$$

where $\lambda=2^{j}, N=0,1,2, \ldots$, and

$$
\bar{\alpha}_{1}=\frac{2}{m+2}+\alpha_{1}=\frac{2}{m+2}+\frac{n}{2}-\frac{1}{m+2}=\frac{n}{2}+\frac{1}{m+2} .
$$

It suffices to prove (4.42) in case $x=0$. In fact, a direct computation yields

$$
\begin{aligned}
& \int_{\mathbb{R}^{n+1}}\left|K_{j}^{\alpha_{1}}(t, 0 ; \tau, y)\right|^{q_{0}} \mathrm{~d} \tau \mathrm{d} y \\
\leq & \int_{-\infty}^{\infty} \int_{\mathbb{R}^{n}} \lambda^{\left(\frac{n+1}{2}-\overline{\alpha_{1}}\right) \cdot q_{0}}\left(|\phi(t)-\phi(\tau)|+\lambda^{-1}\right)^{-\frac{n-1}{2} \cdot q_{0}}(1+\lambda|| y|-| \phi(t)-\phi(\tau)||)^{-N} \mathrm{~d} s \mathrm{~d} y \\
\leq & C \int_{-\infty}^{\infty} \lambda^{\frac{m}{2(m+2)} \cdot q_{0}}\left(|\phi(t)-\phi(\tau)|+\lambda^{-1}\right)^{-\frac{n-1}{2} \cdot q_{0}} \lambda^{-1}\left(|\phi(t)-\phi(\tau)|+\lambda^{-1}\right)^{n-1} \mathrm{~d} \tau \\
\leq & C \int_{-\infty}^{\infty} \lambda^{\frac{m(m+2) n+2 m}{(m+2)((m+2) n-2)}-1}\left(|t-\tau|+\lambda^{-\frac{2}{m+2}}\right)^{-\frac{2(n-1)(m+2)}{(m+2) n-2}} \mathrm{~d} \tau \\
\leq & C .
\end{aligned}
$$

Thus, by interpolation, 4.39) and then further 4.33) follow. 
Relying on Theorem 4.1 and Theorem 4.3, we have:

Lemma 4.5. Let $w$ solve (4.3). Then

$$
\|w\|_{L^{q}\left(\mathbb{R}_{+}^{1+n}\right)}+\left\|\left|D_{x}\right|^{\gamma-\frac{1}{m+2}} w\right\|_{L^{q_{0}\left(\mathbb{R}_{+}^{1+n}\right)}} \leq C\left\|\left|D_{x}\right|^{\gamma-\frac{1}{m+2}} F\right\|_{L^{q^{\prime}\left(\mathbb{R}_{+}^{1+n}\right)}},
$$

where $\gamma=\frac{n}{2}-\frac{(m+2) n+2}{q(m+2)}, q_{0} \leq q<\infty$, and the constant $C$ only depends on $m$, $n$, and $q$.

Proof. Note that

$$
\left(\partial_{t}^{2}-t^{m} \Delta\right)\left|D_{x}\right|^{\gamma-\frac{1}{m+2}} w=\left|D_{x}\right|^{\gamma-\frac{1}{m+2}} F .
$$

Then applying Theorem 4.3 with $q=q_{0}$ yields

$$
\left\|\left|D_{x}\right|^{\gamma-\frac{1}{m+2}} w\right\|_{L^{q_{0}\left(\mathbb{R}_{+}^{1+n}\right)}} \leq C\left\|\left|D_{x}\right|^{\gamma-\frac{1}{m+2}} F\right\|_{L^{q_{0}^{\prime}\left(\mathbb{R}_{+}^{1+n}\right)}} .
$$

Together with Theorem 4.1 this gives 4.44).

\subsection{Proof of Theorem 1.2}

Based on the results of Section 4.1 and 4.2, here we shall prove Theorem 1.2. To establish the existence of a global solution of (1.4), we shall use the iteration scheme

$$
\left\{\begin{array}{l}
\partial_{t}^{2} u_{k}-t^{m} \Delta u_{k}=\left|u_{k-1}\right|^{p}, \\
u_{k}(0, \cdot)=u_{0}(x), \quad \partial_{t} u_{k}(0, \cdot)=u_{1}(x),
\end{array}\right.
$$

where $u_{-1} \equiv 0$.

Proof of Theorem 1.2 We divide the proof into two parts.

\subsubsection{The case when $n \geq 3, p$ is small or $n=2$.}

We will show that there is a solution $u \in L^{r}\left(\mathbb{R}_{+}^{1+n}\right)$ of $(1.4)$ with $r=\left(\frac{m+2}{2} n+1\right) \frac{p-1}{2}$ such that $u_{k} \rightarrow u$ and $\left|u_{k}\right|^{p} \rightarrow|u|^{p}$ in $\mathcal{D}^{\prime}\left(\mathbb{R}_{+}^{1+n}\right)$ as $k \rightarrow \infty$.

We have that $\frac{1}{m+2} \leq \gamma=\frac{n}{2}-\frac{(m+2) n+2}{r(m+2)} \leq 1+\frac{1}{m+2}$ (using $r \geq q_{0}$ ). Set

$$
M_{k}=\left\|u_{k}\right\|_{L^{r}\left(\mathbb{R}_{+}^{1+n}\right)}+\left\|\left|D_{x}\right|^{\gamma-\frac{1}{m+2}} u_{k}\right\|_{L^{q_{0}\left(\mathbb{R}_{+}^{1+n}\right)}} .
$$

Suppose that we have already shown that, for $l=1,2, \ldots, k$,

$$
M_{l} \leq 2 M_{0} \leq C \epsilon_{0}
$$


Then we prove that 4.47) also holds for $l=k+1$. Applying Lemma 4.5 to the equation

$$
\left(\partial_{t}^{2}-t^{m} \Delta\right)\left(u_{k+1}-u_{0}\right)=F\left(u_{k}\right)
$$

where $F\left(u_{k}\right)=\left|u_{k}\right|^{p}$, we arrive at

$$
\begin{aligned}
M_{k+1} & \leq C\left\|\left|D_{x}\right|^{\gamma-\frac{1}{m+2}}\left(F\left(u_{k}\right)\right)\right\|_{L^{q_{0}^{\prime}\left(\mathbb{R}_{+}^{1+n}\right)}}+M_{0} \\
& \leq C\left\|F^{\prime}\left(u_{k}\right)\right\|_{L} L^{\frac{(m+2) n+2}{4}}\left(\mathbb{R}_{+}^{1+n}\right) \\
& \leq C\left\|\left.D_{x}\right|^{\gamma-\frac{1}{m+2}} u_{k}\right\|_{L^{q_{0}}\left(\mathbb{R}_{+}^{1+n}\right)}+M_{L^{\frac{(m+2) n+2}{4}}} M_{\left(\mathbb{R}_{+}^{1+n}\right)} M_{k}+M_{0} .
\end{aligned}
$$

We mention that in this computation the following Leibniz rule for fractional derivatives has been used (see [21, 23] for details):

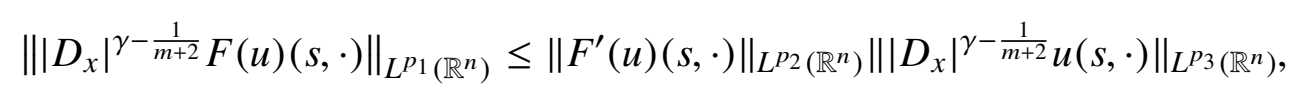

where $\frac{1}{p_{1}}=\frac{1}{p_{2}}+\frac{1}{p_{3}}$ with $p_{i} \geq 1(1 \leq i \leq 3)$ and $0 \leq \gamma-\frac{1}{m+2} \leq 1$. Moreover, it follows from Hölder's inequality that

$$
\left\|F^{\prime}\left(u_{k}\right)\right\|_{L^{\frac{(m+2) n+2}{4}}\left(\mathbb{R}_{+}^{1+n}\right)} \leq C\left\|u_{k}\right\|_{L^{r}\left(\mathbb{R}_{+}^{1+n}\right)}^{p-1} \leq C M_{k}^{p-1} \leq C\left(2 M_{0}\right)^{p-1} .
$$

Thus, if $M_{0} \leq C \epsilon_{0}$ and $\epsilon_{0}$ is so small that

$$
C\left(2 M_{0}\right)^{p-1} \leq \tilde{C} \epsilon_{0}^{p-1} \leq \frac{1}{2}
$$

then we have

$$
M_{k+1} \leq \frac{1}{2} M_{k}+M_{0} \leq 2 M_{0} .
$$

Next we estimate $M_{0}$. By Theorem 4.1, we have that

$$
M_{0} \leq C\left(\|f\|_{\dot{H}^{s}\left(\mathbb{R}^{n}\right)}+\|g\|_{\dot{H}^{s-\frac{2}{m+2}\left(\mathbb{R}^{n}\right)}}\right) \leq C \epsilon_{0},
$$

where $s=\frac{n}{2}-\frac{(m+2) n+2}{(m+2) r}$ and $q_{0} \leq r<\infty$. Therefore, we have obtained the uniform boundedness of the sequences $\left\{M_{k}\right\}$.

Next we show that the sequence $\left\{u_{k}\right\}$ is convergent under the norm $\|\cdot\|_{L^{q_{0}\left(\mathbb{R}_{+}^{1+n}\right)}}$. Set $N_{k}=\left\|u_{k}-u_{k-1}\right\|_{L^{q_{0}\left(\mathbb{R}_{+}^{1+n}\right)}}$. Then

$$
\begin{aligned}
& N_{k+1}=\left\|u_{k+1}-u_{k}\right\|_{L^{q_{0}\left(\mathbb{R}_{+}^{1+n}\right)}} \leq\left\|F\left(u_{k}\right)-F\left(u_{k-1}\right)\right\|_{L^{q^{\prime}}\left(\mathbb{R}_{+}^{1+n}\right)} \\
& \leq\left(\left\|u_{k}\right\|_{L^{r}\left(\mathbb{R}_{+}^{1+n}\right)}+\left\|u_{k-1}\right\|_{L^{r}\left(\mathbb{R}_{+}^{1+n}\right)}\right)^{p-1}\left\|u_{k}-u_{k-1}\right\|_{L^{q_{0}\left(\mathbb{R}_{+}^{1+n}\right)}}
\end{aligned}
$$

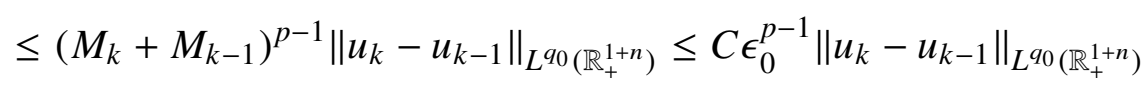

$$
\begin{aligned}
& \leq \frac{1}{2}\left\|u_{k}-u_{k-1}\right\|_{L^{q_{0}\left(\mathbb{R}_{+}^{1+n}\right)}}=\frac{1}{2} N_{k}
\end{aligned}
$$


Therefore, $u_{k} \rightarrow u$ in $L^{q_{0}}\left(\mathbb{R}_{+}^{1+n}\right)$ and hence in $\mathcal{D}^{\prime}\left(\mathbb{R}_{+}^{1+n}\right)$. This yields that there exists a subsequence, which is still denoted by $\left\{u_{k}\right\}$, such that $u_{k} \rightarrow u$ a.e. In view of $\left\|u_{k}\right\|_{L^{r}\left(\mathbb{R}_{+}^{1+n}\right)} \leq 2 M_{0}$, it follows from Fatou's lemma that

$$
\|u\|_{L^{r}\left(\mathbb{R}_{+}^{1+n}\right)} \leq \liminf _{k \rightarrow \infty}\left\|u_{k}\right\|_{L^{r}\left(\mathbb{R}_{+}^{1+n}\right)} \leq 2 M_{0} \leq C \epsilon_{0}<\infty
$$

In order to show that $u$ is a solution of (1.4), It remains to prove that $F\left(u_{k}\right) \rightarrow F(u)$ in $\mathcal{D}^{\prime}\left(\mathbb{R}_{+}^{1+n}\right)$. In fact, for any fixed compact set $K \subseteq \mathbb{R}_{+}^{1+n}$, one has

$$
\begin{aligned}
\left\|F\left(u_{k}\right)-F(u)\right\|_{L^{1}(K)} & \leq C_{K}\left\|F\left(u_{k}\right)-F(u)\right\|_{L^{q^{\prime}(K)}} \\
& \leq C_{K}\left(\left\|u_{k}\right\|_{L^{r}\left(\mathbb{R}_{+}^{1+n}\right)}+\|u\|_{L^{r}(K)}\right)^{p-1}\left\|u_{k}-u\right\|_{L^{q_{0}(K)}} \\
& \leq \tilde{C_{K}} \epsilon_{0}^{p-1}\left\|u_{k}-u\right\|_{L^{q_{0}(K)}} \rightarrow 0 \quad \text { as } \quad k \rightarrow \infty .
\end{aligned}
$$

Thus $\left|u_{k}\right|^{p} \rightarrow|u|^{p}$ in $L_{\text {loc }}^{1}\left(\mathbb{R}_{+}^{1+n}\right)$ and hence in $\mathcal{D}^{\prime}\left(\mathbb{R}_{+}^{1+n}\right)$.

The proof of Subsection 4.3.1 is complete.

\subsubsection{The case when $n \geq 3, p$ is large}

We will show that there is a solution $u \in L^{r}\left(\mathbb{R}_{+}^{1+n}\right)$ of (1.4) with $r=\left(\frac{m+2}{2} n+1\right) \frac{p-1}{2}$ such that $u_{k} \rightarrow u$ and $u_{k}^{p} \rightarrow u^{p}$ in $\mathcal{D}^{\prime}\left(\mathbb{R}_{+}^{1+n}\right)$ as $k \rightarrow \infty$.

We have that $\gamma=\frac{n}{2}-\frac{(m+2) n+2}{(m+2) r}>1+\frac{1}{m+2}$. Let

$$
M_{k}=\sup _{q_{0} \leq q \leq r}\left\|\left|D_{x}\right|^{\frac{(m+2) n+2}{q(m+2)}-\frac{2}{m+2} \cdot \frac{2}{p-1}} u_{k}\right\|_{L^{q\left(\mathbb{R}_{+}^{1+n}\right)}} .
$$

Applying Lemma 4.5 to the equation

$$
\left(\partial_{t}^{2}-t^{m} \Delta\right)\left(u_{k+1}-u_{0}\right)=\left|u_{k}\right|^{p}
$$

yields

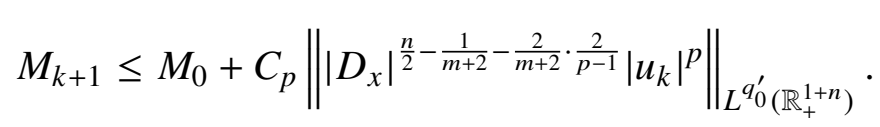

To treat the second summand on the right-hand side of (4.55), we need the following variant of (4.49) (see [14] for details):

$$
\left\|\left|D_{x}\right|^{\sigma}(f g)\right\|_{L^{p}} \leq C\left\|\left|D_{x}\right|^{\sigma} f\right\|_{L^{r_{1}}}\|g\|_{L^{r_{2}}}+C\|f\|_{L^{s_{1}}}\left\|\left|D_{x}\right|^{\sigma} g\right\|_{L^{s_{2}}}
$$

where $0 \leq \sigma \leq 1,1<r_{j}, s_{j}<\infty$, and $\frac{1}{p}=\frac{1}{r_{1}}+\frac{1}{r_{2}}=\frac{1}{s_{1}}+\frac{1}{s_{2}}$.

By (4.56) together with the fact that, for a given multi-index $\alpha$ and $1<p<\infty$,

$$
\left\|D_{x}^{\alpha} f\right\|_{L^{p}} \leq C_{p, \alpha}\left\|\left|D_{x}\right|^{|\alpha|} f\right\|_{L^{p}}
$$


we arrive at

$$
\left\|\left|D_{x}\right|^{\frac{n}{2}-\frac{1}{m+2}-\frac{2}{m+2} \frac{2}{p-1}}\left(\left|u_{k}\right|^{p}\right)\right\|_{L^{p_{0}\left(\mathbb{R}_{+}^{1+n}\right)}} \leq C \prod_{j=1}^{p}\left\|\left|D_{x}\right|^{\alpha_{j}} u_{k}\right\|_{L^{q_{j}\left(\mathbb{R}_{+}^{1+n}\right)}},
$$

where $0 \leq \alpha_{j} \leq \frac{n}{2}-\frac{1}{m+2}-\frac{2}{m+2} \frac{2}{p-1}$ and

$$
\sum_{j=1}^{p} \alpha_{j}=\frac{n}{2}-\frac{1}{m+2}-\frac{2}{m+2} \frac{2}{p-1}
$$

Let $q_{0} \leq q_{j}<\infty$ satisfy

$$
\sum_{j=1}^{p} \frac{1}{q_{j}}=\frac{1}{q_{0}^{\prime}}
$$

where $q_{j}$ is determined by

$$
\frac{(m+2) n+2}{q_{j}(m+2)}-\frac{2}{m+2} \frac{2}{p-1}=\alpha_{j} .
$$

From this, we have

$$
q_{0} \leq q_{j} \leq \frac{(m+2) n+2}{4}(p-1)
$$

and

$$
\begin{aligned}
\sum_{j=1}^{p} \frac{1}{q_{j}} & =\frac{m+2}{(m+2) n+2} \sum_{j=1}^{p}\left(\alpha_{j}+\frac{2}{m+2} \cdot \frac{2}{p-1}\right) \\
& =\frac{m+2}{(m+2) n+2}\left(\frac{n}{2}-\frac{1}{m+2}-\frac{2}{m+2} \frac{2}{p-1}+\frac{2 p}{m+2} \frac{2}{p-1}\right) \\
& =\frac{1}{q_{0}^{\prime}} .
\end{aligned}
$$

Thus one has from 4.55) that

$$
M_{k+1} \leq M_{0}+C_{p} M_{k}^{p}
$$

Suppose that $M_{k} \leq 2 M_{0} \leq C \epsilon_{0}$ holds. Then

$$
M_{k+1} \leq M_{0}+C_{p}\left(2 M_{0}\right)^{p-1} M_{k} \leq M_{0}+\tilde{C}_{p} \epsilon_{0}^{p-1} M_{k}
$$

If $\epsilon_{0}>0$ is so small that $\tilde{C}_{p} \epsilon_{0}^{p-1} \leq 1 / 2$, then

$$
M_{k+1} \leq M_{0}+\frac{1}{2} M_{k} \leq M_{0}+\frac{1}{2} \cdot 2 M_{0}=2 M_{0} .
$$

Thus, we have obtained the uniform boundedness of the $M_{k}$ provided that $M_{0} \leq C \epsilon_{0}$. 
Furthermore, we then have that, if $N_{k}$ is defined as in (4.8),

$$
\begin{aligned}
& N_{k+1}=\left\|u_{k+1}-u_{k}\right\|_{L^{q_{0}\left(\mathbb{R}_{+}^{1+n}\right)}} \\
& \leq||\left|u_{k}\right|^{p}-\left|u_{k-1}\right|^{p} \|_{L^{p_{0}\left(\mathbb{R}_{+}^{1+n}\right)}} \\
& \leq\left(\left\|u_{k}\right\|_{L^{r}\left(\mathbb{R}_{+}^{n+1}\right)}+\left\|u_{k-1}\right\|_{L^{r}\left(\mathbb{R}_{+}^{1+n}\right)}\right)^{p-1}\left\|u_{k}-u_{k-1}\right\|_{L^{q_{0}\left(\mathbb{R}_{+}^{1+n}\right)}} \\
& \leq\left(\sup _{q_{0} \leq q \leq r}\left\|\left|D_{x}\right|^{\frac{(m+2) n+2}{q(m+2)}-\frac{2}{m+2} \cdot \frac{2}{p-1}} u_{k}\right\|_{L^{q}\left(\mathbb{R}_{+}^{1+n}\right)}\right. \\
& \left.+\sup _{q_{0} \leq q \leq r}\left\|\left|D_{x}\right|^{\frac{(m+2) n+2}{q(m+2)}-\frac{2}{m+2} \cdot \frac{2}{p-1}} u_{k-1}\right\|_{L^{q}\left(\mathbb{R}_{+}^{n+1}\right)}\right)^{p-1}\left\|u_{k}-u_{k-1}\right\|_{L^{q_{0}\left(\mathbb{R}_{+}^{1+n}\right)}} \\
& \leq\left(M_{k}+M_{k-1}\right)^{p-1}\left\|u_{k}-u_{k-1}\right\|_{L^{q_{0}\left(\mathbb{R}_{+}^{1+n}\right)}} \\
& \leq C \epsilon_{0}^{p-1}\left\|u_{k}-u_{k-1}\right\|_{L^{q_{0}}\left(\mathbb{R}_{+}^{1+n}\right)} \\
& \leq \frac{1}{2}\left\|u_{k}-u_{k-1}\right\|_{L^{q_{0}\left(\mathbb{R}_{+}^{1+n}\right)}}=\frac{1}{2} N_{k} \text {. }
\end{aligned}
$$

Thus, $u_{k} \rightarrow u$ in $L^{q_{0}}\left(\mathbb{R}_{+}^{1+n}\right)$ as $k \rightarrow \infty$. From here we can finish the proof of Subsection 4.3.2 as in Subsection 4.3 .1 .

Subsection 4.3.1 and Subsection 4.3.2 jointly constitute the proof of Theorem 1.2 



\section{Weighted Strichartz estimate for the homogeneous equation when $\mathbf{p}<\mathbf{p}_{0}$}

The method in Chapter 4 does not work if $p<p_{0}$. This is due to the range of the index in the Strichartz estimate. Indeed, if we want to apply standard iteration as before, we shall need to use the Hölder inequality. If $p<p_{0}$, however, usage of the Hölder inequality introduces a term $T^{\alpha}$ for some positive $\alpha$. When $T$ is small, this factor does not cause trouble and we get local existence. However, when $T$ is large, this factor is hard to control. To overcome this difficulty, we get an $L^{p}-L^{q}$ estimate on $\mathbb{R}_{+}^{1+n}$. To this end, we first establish a pointwise estimate for solutions of linear homogeneous equation. From this pointwise estimate we shall see that the solution behaviors like $(1+\phi(t)|\phi(t)-| x||)^{\alpha}$ for some $\alpha<0$. This fact motivate us to consider the Strichartz estimates with characteristic weight $\phi(t)^{2}-|x|^{2}$.

\subsection{The pointwise estimate}

We start by proving the following pointwise estimate:

Lemma 5.1. Let $v$ solve (4.2). Then

$$
\begin{aligned}
|v(t, x)| \leq & C_{m, n, \delta}(1+\phi(t))^{-\frac{n-1}{2}-\frac{m}{2(m+2)}}(1+|| x|-\phi(t)|)^{-\frac{n}{2}-\frac{1}{m+2}+\delta} \\
& \times\left(\|f\|_{W^{\frac{n}{2}+\frac{1}{m+2}+\delta, 1}\left(\mathbb{R}^{n}\right)}+\|g\|_{W^{\frac{n}{2}-\frac{1}{m+2}+\delta, 1}\left(\mathbb{R}^{n}\right)}\right)
\end{aligned}
$$

for $\delta>0$.

Proof. As in Chapter 4, we may assume that $g=0$. By our analysis there, we can write

$$
\begin{aligned}
v(t, x)=V_{1}\left(t, D_{x}\right) f(x)=C_{m}\left(\int_{\mathbb{R}^{n}} e^{i[x \cdot \xi+\phi(t)|\xi|]} a_{1}(t, \xi) \hat{f}(\xi) \mathrm{d} \xi\right. & \\
& \left.+\int_{\mathbb{R}^{n}} e^{i[x \cdot \xi-\phi(t)|\xi|]} a_{2}(t, \xi) \hat{f}(\xi) \mathrm{d} \xi\right),
\end{aligned}
$$


where $a_{l}(l=1,2)$ satisfies

$$
\left|\partial_{\xi}^{\beta} a_{l}(t, \xi)\right| \leq C_{l \beta}(1+\phi(t)|\xi|)^{-\frac{m}{2(m+2)}}|\xi|^{-|\beta|} .
$$

To estimate $V_{1}\left(t, D_{x}\right) f(x)$, it suffices to deal with the form $\int_{\mathbb{R}^{n}} e^{i[x \cdot \xi+\phi(t)|\xi|]} a_{1}(t, \xi) \hat{f}(\xi) \mathrm{d} \xi$, since the term $\int_{\mathbb{R}^{n}} e^{i[x \cdot \xi-\phi(t)|\xi|]} a_{2}(t, \xi) \hat{f}(\xi) \mathrm{d} \xi$ can be treated analogously. Set

$$
(A f)(t, x)=\int_{\mathbb{R}^{n}} e^{i[x \cdot \xi+\phi(t)|\xi|]} a_{1}(t, \xi) \hat{f}(\xi) \mathrm{d} \xi .
$$

Let $\beta(\tau) \in C_{0}^{\infty}(1 / 2,2)$ such that

$$
\sum_{j=-\infty}^{\infty} \beta\left(2^{-j} \tau\right)=1 \quad \text { for } \tau \in \mathbb{R}_{+} .
$$

To estimate $(A f)(t, x)$, we now study the dyadic operators

$$
\begin{aligned}
A_{j} f(t, x) & =\int_{\mathbb{R}^{n}} e^{i[x \cdot \xi+\phi(t)|\xi|]} \beta\left(2^{-j}|\xi|\right) a_{1}(t, \xi) \hat{f}(\xi) \mathrm{d} \xi \\
& =\int_{\mathbb{R}^{n}} e^{i[x \cdot \xi+\phi(t)|\xi|]} a_{j}(t, \xi) \hat{f}(\xi) \mathrm{d} \xi
\end{aligned}
$$

where $j \in \mathbb{Z}$. Note that the kernel of operator $A_{j}$ is

$$
K_{j}(t, x ; y)=\int_{\mathbb{R}^{n}} e^{i[(x-y) \cdot \xi+\phi(t)|\xi|]} a_{j}(t, \xi) \mathrm{d} \xi,
$$

where $|y| \leq M-1$ because of supp $f \subseteq\{x:|x| \leq M-1\}$. By (3.29) of [15], we have that for any $N \in \mathbb{R}^{+}$,

$$
\begin{aligned}
\left|K_{j}(t, x ; y)\right| \leq C_{m, n, N} \lambda_{j}^{\frac{n+1}{2}}\left(1+\phi(t) \lambda_{j}\right)^{-\frac{m}{2(m+2)}} & \\
& \left(\phi(t)+\lambda_{j}^{-1}\right)^{-\frac{n-1}{2}}\left(1+\lambda_{j}|| x-y|-\phi(t)|\right)^{-N},
\end{aligned}
$$

where $\lambda_{j}=2^{j}$. Since the solution $v$ of (4.2) is smooth and has compact support with respect to the variable $x$ for any fixed time, one easily obtains that 5.1 holds in any domain $[0, T] \times \mathbb{R}^{n}$. Therefore, in order to prove $(5.1)$, it suffices to consider the case $\phi(t) \geq C_{0} M$, where $C_{0}$ is a fixed large constant. From now on, we assume that $|y| \leq M-1$ and $(t, x)$ is in the support of the solution $v$ of (4.2). Next we distinguish two cases.

\subsubsection{The case || $\mathbf{x}-\mathbf{y}|-\phi(\mathbf{t})| \geq \mathbf{C}_{\mathbf{0}} \mathbf{M}$}

In this case, there exist two positive constants $C_{1}$ and $C_{2}$ such that

$$
C_{1}|| x-y|-\phi(t)| \geq|| x|-\phi(t)| \geq C_{2}|| x-y|-\phi(t)| \geq C_{0} M .
$$


For $j \geq 0$, we take $N=\frac{n}{2}+\frac{1}{m+2}+\delta$ in 5.2) to obtain

$$
\begin{aligned}
\left|K_{j}(t, x ; y)\right| & \leq C_{m, n, \delta} \lambda_{j}^{\frac{n+1}{2}-\frac{m}{2(m+2)}} \phi(t)^{-\frac{n-1}{2}-\frac{m}{2(m+2)}} \lambda_{j}^{-\frac{n}{2}-\frac{1}{m+2}-\delta}|| x|-\phi(t)|^{-\frac{n}{2}-\frac{1}{m+2}-\delta} \\
& \leq C_{m, n, \delta} \lambda_{j}^{-\delta}(1+\phi(t))^{-\frac{n-1}{2}-\frac{m}{2(m+2)}}(1+|| x|-\phi(t)|)^{-\frac{n}{2}-\frac{1}{m+2}-\delta} .
\end{aligned}
$$

For $j<0$, taking $N=n / 2+1 /(m+2)-\delta$ in $(5.2)$ we arrive at

$$
\begin{aligned}
\left|K_{j}(t, x ; y)\right| & \leq C_{m, n, \delta} \lambda_{j}^{\frac{n+1}{2}-\frac{m}{2(m+2)}} \phi(t)^{-\frac{n-1}{2}-\frac{m}{2(m+2)}} \lambda_{j}^{-\frac{n}{2}-\frac{1}{m+2}+\delta}|| x|-\phi(t)|^{-\frac{n}{2}-\frac{1}{m+2}+\delta} \\
& \leq C_{m, n, \delta} \lambda_{j}^{\delta}(1+\phi(t))^{-\frac{n-1}{2}-\frac{m}{2(m+2)}}(1+|| x|-\phi(t)|)^{-\frac{n}{2}-\frac{1}{m+2}+\delta} .
\end{aligned}
$$

It follows from $f(x) \in C_{0}^{\infty}\left(\mathbb{R}^{n}\right)$ and direct computation that

$$
\left|A_{j} f\right| \leq \begin{cases}C_{m, n, \delta} \lambda_{j}^{\delta}(1+\phi(t))^{-\frac{n-1}{2}-\frac{m}{2(m+2)}}(1+|| x|-\phi(t)|)^{-\frac{n}{2}-\frac{1}{m+2}+\delta}, & j<0, \\ C_{m, n, \delta} \lambda_{j}^{-\delta}(1+\phi(t))^{-\frac{n-1}{2}-\frac{m}{2(m+2)}}(1+|| x|-\phi(t)|)^{-\frac{n}{2}-\frac{1}{m+2}-\delta}, \quad j \geq 0 .\end{cases}
$$

Summing up the right sides of $\left[5.3\right.$, we get that for large $\phi(t) \geq C_{0} M$ and ||$x|-\phi(t)| \geq C_{0} M$,

$$
|v(t, x)| \leq C_{m, n, \delta}(1+\phi(t))^{-\frac{n-1}{2}-\frac{m}{2(m+2)}}(1+|| x|-\phi(t)|)^{-\frac{n}{2}-\frac{1}{m+2}+\delta} .
$$

\subsubsection{The case || $\mathbf{x}-\mathbf{y}|-\phi(\mathbf{t})| \leq \mathbf{C}_{0} \mathbf{M}$}

By a similar method as in Subsection 5.1.1, we obtain that, for $t>1$,

$$
\|v(t, \cdot)\|_{L^{\infty}\left(\mathbb{R}^{n}\right)} \leq C_{m, n, \delta} \phi(t)^{-\frac{n-1}{2}-\frac{m}{2(m+2)}}\|f\|_{W^{\frac{n}{2}+\frac{1}{m+2}+\delta, 1}\left(\mathbb{R}^{n}\right)}
$$

where $0<\delta<n / 2+1 /(m+2)-\gamma-1 / q$ is a positive constant.

Indeed, note that

$$
\left|A_{j} f(t, x)\right|=\left|\int_{\mathbb{R}^{n}} e^{i[x \cdot \xi+\phi(t) \mid \xi]]} \frac{a_{j}(t, \xi)}{|\xi|^{\alpha}} \overline{\left|D_{x}\right|^{\alpha} f}(\xi) \mathrm{d} \xi\right|,
$$

where $\alpha=n / 2+1 /(m+2)+\delta$. Then, by the stationary phase method, we have that, for $j \geq 0$

$$
\begin{aligned}
\left|A_{j} f\right| & \leq C_{m, n, \delta} \lambda_{j}^{-\alpha} \lambda_{j}^{\frac{n+1}{2}}\left(1+\phi(t) \lambda_{j}\right)^{-\frac{m}{2(m+2)}}\left(\phi(t)+\lambda_{j}^{-1}\right)^{-\frac{n-1}{2}}\|f\|_{W^{\frac{n}{2}+\frac{1}{m+2}+\delta, 1}\left(\mathbb{R}^{n}\right)} \\
& \leq C_{m, n, \delta} \lambda_{j}^{-\delta}(1+\phi(t))^{-\frac{n-1}{2}-\frac{m}{2(m+2)}}\|f\|_{W^{\frac{n}{2}+\frac{1}{m+2}+\delta, 1}\left(\mathbb{R}^{n}\right)} .
\end{aligned}
$$

Similarly, for $j<0$, we have

$$
\left|A_{j} f\right| \leq C_{m, n, \delta} \lambda_{j}^{\delta}(1+\phi(t))^{-\frac{n-1}{2}-\frac{m}{2(m+2)}}\|f\|_{W^{\frac{n}{2}+\frac{1}{m+2}-\delta, 1}\left(\mathbb{R}^{n}\right)} .
$$

Summing up all terms in (5.6) and (5.7) yields

$$
\|v\|_{L^{\infty}\left(\mathbb{R}^{n}\right)} \leq C_{m, n, \delta} \phi(t)^{-\frac{n-1}{2}-\frac{m}{2(m+2)}}\|f\|_{W^{\frac{n}{2}+\frac{1}{m+2}+\delta, 1}\left(\mathbb{R}^{n}\right)},
$$

which shows that (5.5) holds.

Therefore, (5.1) follows from (5.4) and (5.5). 


\subsection{Weighted Strichartz estimate}

Now we can prove the main theorem in this chapter:

Theorem 5.2. For the solution $v$ of (4.2),

$$
\begin{aligned}
& \left\|\left((\phi(t)+M)^{2}-|x|^{2}\right)^{\gamma} v\right\|_{L^{q}\left(\mathbb{R}_{+}^{n+1}\right)} \\
& \quad \leq C\left(\|f\|_{W^{\frac{n}{2}+\frac{1}{m+2}+\delta, 1}\left(\mathbb{R}^{n}\right)}+\|g\|_{W^{\frac{n}{2}-\frac{1}{m+2}+\delta, 1}\left(\mathbb{R}^{n}\right)}\right),
\end{aligned}
$$

where $\phi(t)=\frac{2}{m+2} t^{\frac{m+2}{2}}, q>\frac{2((m+2) n-m)}{(m+2) n-2}, 0<\gamma<\frac{(m+2) n-2}{2(m+2)}-\frac{(m+2) n-m}{(m+2) q}$, and any $0<\delta<$ $n / 2+1 /(m+2)-\gamma-1 / q$, and $C$ is a positive constant depending on $m, n, q, \gamma$ and $\delta$.

Proof. From now on we denote $M_{f, g}=\|f\|_{W^{\frac{n}{2}+\frac{1}{m+2}+\delta, 1}\left(\mathbb{R}^{n}\right)}+\|g\|_{W^{\frac{n}{2}-\frac{1}{m+2}+\delta, 1}\left(\mathbb{R}^{n}\right)}$. We shall compute the integral in (5.8) by using (5.1) and polar coordinates:

$$
\begin{aligned}
& \left\|\left((\phi(t)+M)^{2}-|x|^{2}\right)^{\gamma} v\right\|_{L^{q}\left(\mathbb{R}_{+}^{1+n}\right)}^{q} \\
& \leq C_{m, n, \delta} M_{f, g} \int_{\mathbb{R}_{+}^{1+n}}\left(\left((\phi(t)+M)^{2}-|x|^{2}\right)^{\gamma}(1+\phi(t))^{-\frac{n-1}{2}-\frac{m}{2(m+2)}}\right. \\
& \left.\quad \times(1+|| x|-\phi(t)|)^{-\frac{n}{2}-\frac{1}{m+2}+\delta}\right)^{q} \mathrm{~d} x \mathrm{~d} t \\
& \leq C_{m, n, \delta} M_{f, g} \int_{0}^{\infty} \int_{0}^{\infty}\left((\phi(t)+M+r)^{\gamma}(\phi(t)+M-r)^{\gamma}(1+\phi(t))^{-\frac{n-1}{2}-\frac{m}{2(m+2)}}\right. \\
& \left.\quad \times(1+|r-\phi(t)|)^{-\frac{n}{2}-\frac{1}{m+2}+\delta}\right)^{q} r^{n-1} \mathrm{~d} r \mathrm{~d} t \\
& \leq C_{m, n, \delta} M_{f, g} \int_{0}^{\infty} \int_{0}^{\infty}\left((1+\phi(t))^{-\frac{n-1}{2}-\frac{m}{2(m+2)}+\gamma}\right. \\
& \left.\quad \times(1+|r-\phi(t)|)^{\gamma-\frac{n}{2}-\frac{1}{m+2}+\delta}\right)^{q} r^{n-1} \mathrm{~d} r \mathrm{~d} t .
\end{aligned}
$$

Notice that by our assumption $\gamma-(n-1) / 2-m /(2(m+2))<(m /(m+2)-n) / q$ holds. Thus, we can choose two constants $\sigma>0$ and $\delta>0$ such that

$$
\gamma-\frac{n-1}{2}-\frac{m}{2(m+2)}<\left(\frac{m}{m+2}-n\right) \frac{1}{q}-\sigma
$$

and

$$
\left(\gamma-\frac{n}{2}-\frac{1}{m+2}+\delta\right) q<-1
$$

It follows that, for some positive constant $\bar{\sigma}>0$, the integral in the last line of (5.9) can be controlled by 


$$
\begin{aligned}
\int_{0}^{\infty} \int_{0}^{\infty} & (1+\phi(t))^{\frac{m}{m+2}-n-\bar{\sigma}}(1+|r-\phi(t)|)^{-1-\bar{\sigma}} r^{n-1} \mathrm{~d} r \mathrm{~d} t \\
& \leq C \int_{0}^{\infty}(1+\phi(t))^{\frac{m}{m+2}-n-\bar{\sigma}}(1+\phi(t))^{n-1} \mathrm{~d} t \\
& \leq C
\end{aligned}
$$

which derives

$$
\left\|\left((\phi(t)+M)^{2}-|x|^{2}\right)^{\gamma} v\right\|_{L^{q}\left(\mathbb{R}_{+}^{n+1}\right)} \leq C_{m, n, \delta} M_{f, g},
$$

and $(5.8)$ is proved. 



\section{Weighted Strichartz estimate for the inhomogeneous equation.}

Since we have already established the weighted Strichartz estimate for the homogeneous equation, what remains to do is to prove the weighted Strichartz estimate for the inhomogeneous equation. From now on, we set $q=p+1$ for the exponent in (1.1). For technical reasons, we shall first give the result in the characteristic cone $\{(t, x)|| x \mid \leq \phi(t)\}$.

Theorem 6.1. For problem (4.3), assume that $F(t, x) \equiv 0$ when $|x|>\phi(t)-1$. Let $p_{c}<p<q_{0}-1$. Then there exist a $\gamma$ satisfying $0<\gamma<\frac{(m+2) n-2}{2(m+2)}-\frac{(m+2) n-m}{(m+2) q}$ and $p \gamma>\frac{1}{q}$ such that

$$
\left\|\left(\phi^{2}(t)-|x|^{2}\right)^{\gamma} w\right\|_{L^{q\left(\mathbb{R}_{+}^{1+n}\right)}} \leq C\left\|\left(\phi^{2}(t)-|x|^{2}\right)^{p \gamma} F\right\|_{L^{\frac{q}{q-1}}\left(\mathbb{R}_{+}^{1+n}\right)},
$$

where $C>0$ is a constant depending on $m, n, q$, and $\gamma$.

More specifically, since $p>p_{c}$, where $p_{c}$ solves (1.8), by a direct computation

$$
\frac{1}{p(p+1)}<\frac{(m+2) n-2}{2(m+2)}-\frac{(m+2) n-m}{(m+2)(p+1)} \text {. }
$$

From now on we set

$$
\gamma_{0}=\frac{(m+2) n-2}{2(m+2)}-\frac{(m+2) n-m}{(m+2)(p+1)} .
$$

Thus, there exist a $v>0$, such that

$$
\gamma_{0}-v<\gamma_{0}
$$

and

$$
\gamma_{0}+v>\frac{1}{p}
$$

Hence, for any fixed $m, n$ and $p$, one can choose a $v>0$ such that

$$
p\left(\gamma_{0}-v\right)=\gamma_{0}+v
$$


and 6.2, 6.3 hold. In fact, one needs to take

$$
v=\frac{p-1}{p+1} \gamma_{0} .
$$

Then Theorem 6.1 is a corollary of the following theorem

Theorem 6.2. For problem (4.3), we assume $F(t, x) \equiv 0$ when $|x|>\phi(t)-1$. Let $1<p<q_{0}-1$. Then there exist a $v>0$ such that

$$
\left\|\left(\phi^{2}(t)-|x|^{2}\right)^{\gamma_{0}-v} w\right\|_{L^{q}\left(\mathbb{R}_{+}^{1+n}\right)} \leq C\left\|\left(\phi^{2}(t)-|x|^{2}\right)^{\gamma_{0}+v} F\right\|_{L^{\frac{q}{q-1}}\left(\mathbb{R}_{+}^{1+n}\right)},
$$

and $C>0$ is a constant depending on $m, n, q$, and $\gamma$.

Before we give the proof of Theorem 6.2, we show that how Theorem 6.1 yields the following result

Theorem 6.3. For problem (4.1), assume that $F(t, x) \equiv 0$ when $|x|>\phi(t)+M-1$ and $F(t, x) \in C^{\infty}\left(\left[0, T_{0}\right] \times \mathbb{R}^{n}\right)$ for some fixed number $T_{0}, 0<T_{0}<1$. Let $p_{c}<p<q_{0}-1$. Then there exist some $\gamma$ satisfying $0<\gamma<\frac{(m+2) n-2}{2(m+2)}-\frac{(m+2) n-m}{(m+2) q}$, $p \gamma>\frac{1}{q}$ such that

$$
\begin{aligned}
& \left\|\left((\phi(t)+M)^{2}-|x|^{2}\right)^{\gamma} w\right\|_{L^{q}\left(\left[T_{0} / 2, \infty\right) \times \mathbb{R}^{n}\right)} \\
& \quad \leq C\left\|\left((\phi(t)+M)^{2}-|x|^{2}\right)^{p \gamma} F\right\|_{L^{\frac{q}{q-1}\left(\left[T_{0} / 2, \infty\right) \times \mathbb{R}^{n}\right)}},
\end{aligned}
$$

where $C>0$ is a constant depending on $m, n, q$, and $\gamma$.

Proof. To prove (6.5), first we consider the case $F(t, x) \equiv 0$ when $|x|>\phi(t)-\phi\left(T_{0} / 4\right)$. Note that the region $\left\{(t, x): t \geq T_{0} / 2,|x| \leq \phi(t)+M-1\right\}$ can be covered by a finite number of cones $\left\{Q_{j}\right\}_{j=1}^{N_{0}}$, where each cone $Q_{j}(j \geq 2)$ is a shift in the $x$ variable with respect to the curved cone

$$
Q_{1}=\left\{(t, x): t \geq T_{0} / 2,|x| \leq \phi(t)-\phi\left(T_{0} / 4\right)\right\} .
$$

Set

$$
\begin{aligned}
& F_{1}=\chi_{Q_{1}} F \\
& \begin{aligned}
F_{2} & =\chi_{Q_{2}}\left(1-\chi_{Q_{1}}\right) F \\
& \quad \cdots \\
F_{N_{0}} & \left.=\chi_{Q_{N_{0}}}\left(1-\chi_{Q_{1}}-\chi_{Q_{2}}\left(1-\chi_{Q_{1}}\right)-\cdots-\chi_{Q_{N_{0}-1}}\left(1-\chi_{Q_{1}}\right)\right) \cdots\left(1-\chi_{Q_{N_{0}-2}}\right)\right) F,
\end{aligned}
\end{aligned}
$$

where $\chi_{Q_{j}}$ stands for the characteristic function of $Q_{j}$, and $\sum_{j=1}^{N_{0}} F_{j}=F$. Let $w_{j}$ solve

$$
\left\{\begin{array}{l}
\partial_{t}^{2} w_{j}-t^{m} \Delta w_{j}=F_{j}(t, x) \\
w_{j}(0, x)=0, \quad \partial_{t} w_{j}(0, x)=0 .
\end{array}\right.
$$


Then supp $w_{j} \subseteq Q_{j}$. Since the Tricomi equation is invariant under the translation with respect to the variable $x$, it follows from Theorem 6.1 that

$$
\left\|\left(\phi^{2}(t)-\left|x-v_{j}\right|^{2}\right)^{\gamma_{1}} w_{j}\right\|_{L^{q}\left(Q_{j}\right)} \leq C\left\|\left(\phi^{2}(t)-\left|x-v_{j}\right|^{2}\right)^{\gamma_{2}} F_{j}\right\|_{L^{\frac{q}{q-1}}\left(Q_{j}\right)},
$$

where $v_{j} \in \mathbb{R}^{n}$ corresponds to the coordinate shift of the space variable $x$ from $Q_{1}$ to $Q_{j}$.

Next we derive (6.5) by using (6.6) and the condition $t \geq T_{0} / 4$. First, we demonstrate that there exists a constant $\delta>0$ such that, for $(t, x) \in Q_{j}$

$$
\phi^{2}(t)-\left|x-v_{j}\right|^{2} \geq \delta\left((\phi(t)+M)^{2}-|x|^{2}\right) .
$$

To establish these inequalities, it suffices to prove (6.7) in two extreme cases: $v_{j}=0$ and $\left|v_{j}\right|=M-1+\phi\left(3 T_{0} / 8\right)$ (we need $\left|v_{j}\right|>M-1$ to cover the whole region $\{(t, x): t \geq$ $\left.\left.T_{0} / 2,|x| \leq \phi(t)+M-1\right\}\right)$.

For $v_{j}=0,6.7$ is equivalent to

$$
\phi^{2}(t) \geq(1-\delta)|x|^{2}+\delta(\phi(t)+M)^{2} .
$$

By $|x| \leq \phi(t)-\phi\left(T_{0} / 4\right)$ for $(t, x) \in Q_{1}$, in order to show (6.8) it suffices to prove

$$
\phi^{2}(t) \geq(1-\delta)\left(\phi(t)-\phi\left(T_{0} / 4\right)\right)^{2}+\delta(\phi(t)+M)^{2}
$$

This is equivalent to

$$
\left\{2(1-\delta) \phi\left(T_{0} / 4\right)-2 \delta M\right\} \phi(t) \geq(1-\delta) \phi^{2}\left(T_{0} / 4\right)+\delta M^{2} .
$$

It is easily achieved by $t \geq T_{0} / 4$ and the smallness of $\delta$.

For $v_{j}=M-1+\phi\left(3 T_{0} / 8\right)$, the computation is a little more involved. First, note that for fixed $t$, the region $\left\{(t, x): t \geq T_{0} / 2,|x| \leq \phi(t)+M-1\right\}$ is symmetric with respect to $x$ variable. Thus we can assume $v_{j}=(v, 0, \ldots, 0)$, where $v=\left|v_{j}\right|=M-1+\phi\left(3 T_{0} / 8\right)$. In this case, setting $x=\left(x_{1}, x^{\prime}\right)$, 6.9) is equivalent to

$$
\begin{aligned}
\phi^{2}(t) & \geq\left|x-v_{j}\right|^{2}+\delta\left((\phi(t)+M)^{2}-|x|^{2}\right) \\
& =(1-\delta) x_{1}^{2}-2 v x_{1}+v^{2}+(1-\delta)\left|x^{\prime}\right|^{2}+\delta(\phi(t)+M)^{2} \\
& =G(t, x) .
\end{aligned}
$$

For fixed $t, \mathrm{G}(\mathrm{t}, \mathrm{x})$ is a hyperbolic paraboloid, and it assumes its minimum at the point $x=(v /(1-\delta), 0)$. Thus for the same fixed t, the maximum of the $G(t, x)$ in $\left\{x|| x-v_{j} \mid \leq\right.$ $\left.\phi(t)-\phi\left(T_{0} / 4\right)\right\}$ must is assumed on the boundary $\left|x-v_{j}\right|=\phi(t)-\phi\left(T_{0} / 4\right)$. Then our task is reduced to prove

$$
\phi^{2}(t) \geq\left(\phi(t)-\phi\left(T_{0} / 4\right)\right)^{2}+\delta\left((\phi(t)+M)^{2}-|x|^{2}\right)
$$


We shall consider the case that $|x|^{2}$ assume its minimum on the boundary. In addition, on the boundary $\left|x-v_{j}\right|=\phi(t)-\phi\left(T_{0} / 4\right)$, we have

$$
|x|^{2}=\left(\phi(t)-\phi\left(T_{0} / 4\right)\right)^{2}+2 v x_{1}-v^{2} .
$$

Then we shall take

$$
x_{1}=v-\phi(t)+\phi\left(T_{0} / 4\right), \quad x^{\prime}=0 .
$$

Substituting (6.13) and (6.12) into 6.11), we are left to prove

$$
\begin{aligned}
\phi^{2}(t) \geq\left(\phi(t)-\phi\left(T_{0} / 4\right)\right)^{2}+ & \delta\left\{(\phi(t)+M)^{2}\right. \\
& \left.-\left(\phi(t)-\phi\left(T_{0} / 4\right)\right)^{2}+2 v\left(\phi(t)-\phi\left(T_{0} / 4\right)\right)-v^{2}\right\} \\
& =\phi^{2}(t)+\left\{2 \delta\left(\phi\left(T_{0} / 4\right)+M+v\right)-2 \phi\left(T_{0} / 4\right)\right\} \phi(t) \\
& +(1-\delta) \phi^{2}\left(T_{0} / 4\right)+\delta M^{2}-\delta v\left(v+2 \phi\left(T_{0} / 4\right)\right) .
\end{aligned}
$$

For fixed $T_{0}$ and $M$, if $\delta$ is small enough, then

$$
2 \delta\left(\phi\left(T_{0} / 4\right)+M+v\right) \leq \frac{1}{2} \phi\left(T_{0} / 4\right)
$$

and

$$
(1-\delta) \phi^{2}\left(T_{0} / 4\right)+\delta M^{2} \leq \frac{3}{2} \phi^{2}\left(T_{0} / 4\right)
$$

Substituting (6.15) and 6.16) into (6.14), our remaining task is to prove

$$
-\frac{3}{2} \phi\left(T_{0} / 4\right) \phi(t)+\frac{3}{2} \phi^{2}\left(T_{0} / 4\right) \leq 0,
$$

but since $t \geq T_{0} / 4$, this holds.

Thus, for $(t, x) \in \bigcup_{j=1}^{N_{0}} Q_{j}$, there exists a positive constant $c>0$ such that, for $1 \leq j \leq N_{0}$,

$$
c\left((\phi(t)+M)^{2}-|x|^{2}\right) \leq \phi^{2}(t)-\left|x-v_{j}\right|^{2} .
$$

On the other hand, by $|x| \leq \phi(t)+M-1$, one has

$$
\begin{aligned}
2\left\{(\phi(t)+M)^{2}-|x|^{2}\right\}-\left\{\phi^{2}(t)-\left|x-v_{j}\right|^{2}\right\} \\
\geq(|x|+1)^{2}-|x|^{2}+(\phi(t)+M)^{2}-|x|^{2}-\phi^{2}(t)+\left|x-v_{j}\right|^{2} \\
=2 M \phi(t)+M^{2}+\left|v_{j}\right|^{2}+1+2\left(1-\left|v_{j}\right|\right)|x| .
\end{aligned}
$$

In addition, if $1-\left|v_{j}\right|<0$, then by $\left|v_{j}\right| \leq M-1+\phi\left(3 T_{0} / 8\right)$ and the smallness of $T_{0}$, the last line in 5.4 is bounded from below by 


$$
\begin{aligned}
2 M \phi(t) & +M^{2}+\left|v_{j}\right|^{2}+1+2\left\{2-M-\phi\left(3 T_{0} / 8\right)\right\}\{\phi(t)+M-1\} \\
& =4 \phi(t)-M^{2}+6 M-3+\left|v_{j}\right|^{2}-2 \phi\left(3 T_{0} / 8\right) \phi(t)-2(M-1) \phi\left(3 T_{0} / 8\right) \\
& \geq 2 \phi(t)-M^{2}+1
\end{aligned}
$$

while in the case $\left.1-\left|v_{j}\right| \geq 0,6.18\right)$ yields

$$
2\left\{(\phi(t)+M)^{2}-|x|^{2}\right\}-\left\{\phi^{2}(t)-\left|x-v_{j}\right|^{2}\right\} \geq M^{2}+1>0 .
$$

Combining (6.19) and (6.20) yields that for $2 \phi(t) \geq M^{2}-1$,

$$
\phi^{2}(t)-\left|x-v_{j}\right|^{2} \leq C\left((\phi(t)+M)^{2}-|x|^{2}\right) .
$$

If $2 \phi(t)<M^{2}-1$, then

$$
\phi^{2}(t)-\left|x-v_{j}\right|^{2} \leq \phi^{2}(t) \leq C_{M} \leq C_{M}\left((\phi(t)+M)^{2}-|x|^{2}\right) .
$$

Thus, it follows from 6.12)-6.13 that, for $j=1, \ldots, N_{0}$,

$$
\phi^{2}(t)-\left|x-v_{j}\right|^{2} \leq C\left((\phi(t)+M)^{2}-|x|^{2}\right) .
$$

Therefore,

$$
\begin{aligned}
\left\|\left((\phi(t)+M)^{2}-|x|^{2}\right)^{\gamma} w\right\|_{L^{q}\left(\left[T_{0} / 2, \infty\right) \times \mathbb{R}^{n}\right)} & \leq C \sum_{j=1}^{N_{0}}\left\|\left((\phi(t)+M)^{2}-|x|^{2}\right)^{\gamma} w_{j}\right\|_{L^{q}\left(Q_{j}\right)} \\
& \leq C \sum_{j=1}^{N_{0}}\left\|\left(\phi^{2}(t)-\left|x-v_{j}\right|^{2}\right)^{\gamma} w_{j}\right\|_{L^{q}\left(Q_{j}\right)} \\
& \leq C \sum_{j=1}^{N_{0}}\left\|\left(\phi^{2}(t)-\left|x-v_{j}\right|^{2}\right)^{p \gamma} F_{j}\right\|_{L^{\frac{q}{q-1}}\left(Q_{j}\right)} \\
& \leq C \sum_{j=1}^{N_{0}}\left\|\left((\phi(t)+M)^{2}-|x|^{2}\right)^{p \gamma} F_{j}\right\|_{L^{\frac{q}{q-1}}\left(Q_{j}\right)} \\
& \leq C_{N_{0}}\left\|\left((\phi(t)+M)^{2}-|x|^{2}\right)^{p \gamma} F\right\|_{L^{\frac{q}{q-1}}\left(\left[T_{0} / 2, \infty\right) \times \mathbb{R}^{n}\right)},
\end{aligned}
$$

which derives 6.5).

Now let us turn to the proof of Theorem 6.2. This proof relies on the validity of two endpoint estimates to be shown the remaining two chapters. 
6 Weighted Strichartz estimate for the inhomogeneous equation.

Proof of Theorem 6.2 To establish (6.1), motivated by [7], we prove in Chapter 7 and Chapter 8 that (6.4) holds for two special cases, namely, 6.4) holds for the two endpoints $q=q_{0}=\frac{2((m+2) n+2)}{(m+2) n-2}$ (corresponding to $\left.\frac{(m+2) n-2}{2(m+2)}-\frac{(m+2) n-m}{(m+2) q}=\frac{1}{q}\right)$ and $q=2$ (corresponding $\left.q=\frac{q}{q-1}\right)$ :

$$
\left\|\left(\phi^{2}(t)-|x|^{2}\right)^{1 / q_{0}-v} w\right\|_{L^{q_{0}\left(\mathbb{R}_{+}^{1+n}\right)}} \leq C\left\|\left(\phi^{2}(t)-|x|^{2}\right)^{1 / q_{0}+v} F\right\|_{L^{\frac{q_{0}}{q_{0}^{-1}}\left(\mathbb{R}_{+}^{1+n}\right)}}
$$

and

$$
\left\|\left(\phi^{2}(t)-|x|^{2}\right)^{\frac{m-2}{2(m+2)}-v} w\right\|_{L^{2}\left(\mathbb{R}_{+}^{1+n}\right)} \leq C\left\|\left(\phi^{2}(t)-|x|^{2}\right)^{1 / 2+v} F\right\|_{L^{2}\left(\mathbb{R}_{+}^{1+n}\right)} .
$$

By 6.24 - 6.25) and interpolation, one gets 6.4. 


\section{$7 \quad L^{q}$ estimate for $q=q_{0}$}

In this chapter, we will give a proof of 6.24). Since (6.24) is a weighted estimate, we shall split up $w$ into pieces supported in regions which scale with the time variable to prove the dyadic estimates first. In fact, one can write inequality (6.24) as

$$
\left\|\left(\phi(t)^{2}-|x|^{2}\right)^{1 / q_{0}-v} w\right\|_{L^{q_{0}\left(\mathbb{R}_{+}^{1+n}\right)}} \leq C\left\|\left(\phi(t)^{2}-|x|^{2}\right)^{1 / q_{0}+v} F\right\|_{L^{\frac{q_{0}}{q_{0}^{-1}}\left(\mathbb{R}_{+}^{1+n}\right)}},
$$

where $v>0$ and $q_{0}=\frac{2((m+2) n+2)}{(m+2) n-2}$. To prove 7.1$)$, it suffices to prove the following inequality, for $T \geq \bar{T}$ while $\bar{T}$ is a fixed large constant,

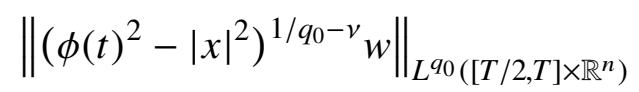

$$
\begin{aligned}
& \leq C \phi(T)^{-v}|\ln T|^{1 / q_{0}}\left\|\left(\phi(t)^{2}-|x|^{2}\right)^{1 / q_{0}+v} F\right\|_{L^{\frac{q_{0}}{q_{0}^{-1}}\left(\mathbb{R}_{+}^{1+n}\right)}} .
\end{aligned}
$$

In fact, Lemma 3.4 of [10] implies, for $t \in[0, \bar{T} / 2]$,

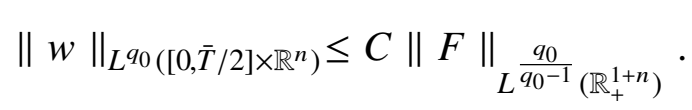

In this case, the weight $\phi(t)^{2}-|x|^{2}$ is bounded from below and above. Thus we have

$$
\begin{aligned}
\left\|\left(\phi(t)^{2}-|x|^{2}\right)^{1 / q_{0}-v} w\right\|_{L^{q_{0}}\left([0, \bar{T} / 2] \times \mathbb{R}^{n}\right)} & \leq C\left\|\left(\phi(t)^{2}-|x|^{2}\right)^{1 / q_{0}+v} F\right\|_{L^{\frac{q_{0}}{q_{0}-1}}\left(\mathbb{R}_{+}^{1+n}\right)} .
\end{aligned}
$$

Summing (7.2) over all the $T \geq \bar{T}$ together with 7.3 yields (7.1). Note that, by Lemma 5.1 , the solution of homogeneous equation (4.2) has a faster decay if $(t, x)$ is far from the characteristic cone $\{(t, x): t>0,|x|=\phi(t)\}$. Motivated by this observation and [7], we shall first establish (7.2) in the set $\{(t, x): t>0,|x| \leq \phi(t) / 2\}$. To this end, we now prove one basic inequality. All other cases are derived utilizing this result.

Proposition 7.1. Assume $F(t, x) \equiv 0$ if $n \geq 3$ and $\phi(t)^{2}-|x|^{2}<1$. Then the solution $w$ of (4.3) satisfies 


$$
\begin{aligned}
\left\|\left(\phi(t)^{2}-|x|^{2}\right)^{1 / q_{0}} w\right\|_{L^{q_{0}}\left(\left\{(t, x): T / 2 \leq t \leq T,|x| \leq \frac{\phi(t)}{2}\right\}\right.} & \\
& \leq C|\ln T|^{1 / q_{0}}\left\|\left(\phi(t)^{2}-|x|^{2}\right)^{1 / q_{0}} F\right\|_{L^{\frac{q_{0}}{q_{0}^{-1}}\left(\mathbb{R}_{+}^{1+n}\right)}},
\end{aligned}
$$

where $C=C(m, n)>0$ is a constant.

Remark 7.2. Note that $\operatorname{supp} F \subseteq\{(t, x)|\phi(t)-| x \mid \geq 1\}$ implies $\operatorname{supp} F \subseteq\{(t, x) \mid$ $\left.\phi(t)^{2}-|x|^{2} \geq 1\right\}$. Thus, Proposition 7.1 is more general than what we need in the proof of Theorem 6.1.

\subsection{Proof of Proposition 7.1}

Proof. Set $w_{T}(t, x)=w\left(T t, T^{\frac{m+2}{2}} x\right)$ and $F_{T}(t, x)=T^{2} F\left(T t, T^{\frac{m+2}{2}} x\right)$. Then

$$
\left(\partial_{t}^{2}-t^{m} \triangle_{x}\right) w_{T}=F_{T}
$$

and, (7.4) is equivalent to

$$
\left\|w_{T}\right\|_{L^{q_{0}}(\{(t, x): 1 / 2 \leq t \leq 1,|x| \leq \phi(t) / 2\}} \leq C|\ln T|^{1 / q_{0}}\left\|\left(\phi(t)^{2}-|x|^{2}\right)^{1 / q_{0}} F_{T}\right\|_{L^{\frac{q_{0}}{q^{-1}}}\left(\mathbb{R}_{+}^{n+1}\right)} .
$$

Note that the advantage of inequality (7.5) is that only right hand side of (7.5) contains a weight.

To prove (7.5), the following two cases will be considered: $\operatorname{supp} F(\cdot, y) \subseteq\{s>$ $0: \phi(s)<(1 / 8) \phi(1 / 2)\}$, and $\operatorname{supp} F(\cdot, y) \subseteq\{s>0: \phi(s) \geq(1 / 8) \phi(1 / 2)\}$ For the first case, we shall derive (7.5) by establishing a pointwise estimate of $w_{T}$. For the second case, (7.5) will be shown by applying some techniques in microlocal analysis and analyzing the solution $w_{T}$ precisely.

\subsubsection{The case $\operatorname{supp} F(\cdot, y) \subseteq\{s>0: \phi(s)<\phi(1 / 2) / 8\}$.}

Note that

$$
w_{T}(t, x)=\int_{0}^{t}\left(V_{2}\left(t, D_{x}\right) V_{1}\left(s, D_{x}\right)-V_{1}\left(t, D_{x}\right) V_{2}\left(s, D_{x}\right)\right) F_{T}(s, x) \mathrm{d} s .
$$

By an analogous analysis as in Lemma 3.4 of [10], we have

$$
w_{T}(t, x)=\int_{0}^{t} \int_{\mathbb{R}^{n}} e^{i[x \cdot \xi+(\phi(t)-\phi(s))|\xi|]} a(t, s, \xi) \hat{F}_{T}(s, \xi) \mathrm{d} \xi \mathrm{d} s,
$$

where the amplitude function $a$ satisfies for $\beta \in \mathbb{N}_{0}^{n}$,

$$
\left|\partial_{\xi}^{\beta} a(t, s, \xi)\right| \leq C(1+\phi(t)|\xi|)^{-\frac{m}{2(m+2)}}(1+\phi(s)|\xi|)^{-\frac{m}{2(m+2)}}|\xi|^{-\frac{2}{m+2}-|\beta|} .
$$


As in the proof of Theorem 4.1, to estimate $w_{T}(t, x)$ in (7.7), we first deal with the dyadic operators

$$
\begin{aligned}
w_{T}^{j}(t, x) & =\int_{0}^{t} \int_{\mathbb{R}^{n}} e^{i[x \cdot \xi+(\phi(t)-\phi(s))|\xi|]} \beta\left(\frac{|\xi|}{\lambda_{j}}\right) a(t, s, \xi) \hat{F}(s, \xi) \mathrm{d} \xi \mathrm{d} s \\
& =\int_{0}^{t} \int_{\mathbb{R}^{n}} K_{j}(t, x ; s, y) F_{T}(s, y) \mathrm{d} y \mathrm{~d} s,
\end{aligned}
$$

where the cut-off function $\beta$ is given in Lemma 3.2, and $\lambda_{j}=2^{j}$ for $j \in \mathbb{Z}$. It follows from Lemma 7.2.4 of [31] (see also (3.41) of [10]) that, for any $N \in \mathbb{R}^{+}$,

$$
\begin{aligned}
\left|K_{j}(t, x ; s, y)\right| \leq C_{m, n, N} \lambda_{j}^{\frac{n+1}{2}-\frac{2}{m+2}}(\mid \phi(t)- & \left.\phi(s) \mid+\lambda_{j}^{-1}\right)^{-\frac{n-1}{2}}\left(1+\phi(t) \lambda_{j}\right)^{-\frac{m}{2(m+2)}} \\
& \times\left(1+\lambda_{j}|| \phi(t)-\phi(s)|-| x-y||\right)^{-N} .
\end{aligned}
$$

For $j \geq 0$, by the compact support of $F_{T}$ and $w_{T}$ in the time variable, we have $(3 / 8) \phi(t) \leq$ $\phi(t)-\phi(s) \leq \phi(t)$ and ||$\phi(t)-\phi(s)|-| x-y|| \geq(1 / 4)|\phi(t)-| x||$. Hence, we can choose $N=n / 2-1 /(m+2)+\delta$ with $\delta>0$ being a small constant such that

$$
\begin{aligned}
\left|K_{j}(t, x ; s, y)\right| & \leq C_{m, n, \delta} \lambda_{j}^{\frac{n+1}{2}-\frac{2}{m+2}} \phi(t)^{-\frac{n-1}{2}}\left(\phi(t) \lambda_{j}\right)^{-\frac{m}{2(m+2)}}\left(\lambda_{j}|\phi(t)-| x||\right)^{-\frac{n}{2}+\frac{1}{m+2}-\delta} \\
& \leq\left. C_{m, n, \delta} \lambda_{j}^{-\delta} \phi(t)^{-\frac{n-1}{2}-\frac{m}{2(m+2)}}|\phi(t)-| x\right|^{-\frac{n}{2}+\frac{1}{m+2}-\delta}
\end{aligned}
$$

Denote $D_{1}=\left\{\left.(s, y)\left|0<s \leq 1 / 8 \phi(1 / 2), \phi(s)^{2}-\right| y\right|^{2} \geq 1 /\left(T^{m+2}\right)\right\}$. By Hölder's inequality,

$$
\begin{aligned}
\left|w_{T}^{j}\right| \leq\left\|K_{j}(t, x ; s, y)\left(\phi^{2}(s)-|y|^{2}\right)^{-1 / q_{0}}\right\|_{L^{q_{0}}\left(D_{1}\right)} & \\
& \times\left\|\left(\phi^{2}(s)-|y|^{2}\right)^{1 / q_{0}} F_{T}(s, y)\right\|_{L^{\frac{q_{0}}{q_{0}-1}\left(D_{1}\right)}} .
\end{aligned}
$$

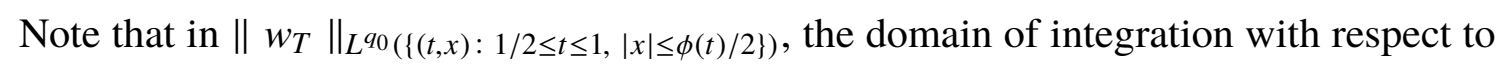
$(t, x)$ is bounded. Then (7.5) can be established if we can bound $\| K_{j}(t, x ; s, y)\left(\phi^{2}(s)-\right.$ $\left.|y|^{2}\right)^{-1 / q_{0}} \|_{L^{q_{0}}\left(D_{1}\right)}$. Now we focus on the treatment of $\left\|K_{j}(t, x ; s, y)\left(\phi^{2}(s)-|y|^{2}\right)^{-1 / q_{0}}\right\|_{L^{q_{0}}\left(D_{1}\right)}$. By the assumption of $1 / 2 \leq t \leq 1$ and $|x| \leq \phi(t) / 2$, one has

$$
\left.\phi(t)^{-\frac{n-1}{2}-\frac{m}{2(m+2)}}|\phi(t)-| x\right|^{-\frac{n}{2}+\frac{1}{m+2}-\delta} \leq C .
$$

In addition, a direct computation yields 


$$
\begin{aligned}
\left\|\left(\phi^{2}(s)-|y|^{2}\right)^{1 / q_{0}}\right\|_{L^{q_{0}\left(D_{1}\right)}}^{q_{0}} & \\
& =\int_{\phi^{-1}(1) / T}^{\phi^{-1}(\phi(1 / 2) / 8)} \int_{\phi(s)^{2}-|y|^{2} \geq \frac{1}{T^{m+2}}} \frac{1}{\phi(s)^{2}-|y|^{2}} \mathrm{~d} y \mathrm{~d} s \\
& \leq C \int_{\phi^{-1}(1) / T}^{\phi^{-1}(\phi(1 / 2) / 8)} \int_{0}^{\sqrt{\phi(s)^{2}-\frac{1}{T^{m+2}}}} \frac{r^{n-1}}{\phi(s)^{2}-r^{2}} \mathrm{~d} r \mathrm{~d} s \\
& \leq C \int_{\phi^{-1}(1) / T}^{\phi^{-1}(\phi(1 / 2) / 8)} \phi(s)^{n-2}(|\ln \phi(s)|+|\ln \phi(T)|) \mathrm{d} s \\
& \leq C|\ln T| .
\end{aligned}
$$

Together with (7.11), we then have that, for $j \geq 0$,

$$
\begin{aligned}
& \left\|w_{T}^{j}\right\|_{L^{q_{0}}(\{(t, x): 1 / 2 \leq t \leq 1,|x| \leq \phi(t) / 2\})} \\
& \quad \leq C \lambda_{j}^{-\delta}|\ln T|^{1 / q_{0}}\left\|\left(\phi(t)^{2}-|x|^{2}\right)^{1 / q_{0}} F_{T}\right\|_{L^{\frac{q_{0}}{q_{0}^{-1}}}\left(\mathbb{R}_{+}^{1+n}\right)} .
\end{aligned}
$$

Similarly, for $j<0$, one has

$$
\begin{aligned}
& \left\|w_{T}^{j}\right\|_{L^{q_{0}}(\{(t, x): 1 / 2 \leq t \leq 1,|x| \leq \phi(t) / 2\})} \\
& \leq C \lambda_{j}^{\delta}|\ln T|^{1 / q_{0}}\left\|\left(\phi(t)^{2}-|x|^{2}\right)^{1 / q_{0}} F_{T}\right\|_{L^{\frac{q_{0}}{q_{0}-1}\left(\mathbb{R}_{+}^{1+n}\right)}} .
\end{aligned}
$$

It follows from Lemma 3.2 that

$\left\|W_{T}\right\|_{L^{q_{0}}(\{(t, x): 1 / 2 \leq t \leq 1,|x| \leq \phi(t) / 2\})}$

$$
\begin{aligned}
& \leq C\left(\sum_{j=-\infty}^{\infty}\left\|w_{T}^{j}\right\|_{L^{q}\left(\left\{(t, x): \frac{1}{2} \leq t \leq 1,|x| \leq \frac{\phi(t)}{2}\right\}\right)}^{2}\right)^{\frac{1}{2}} \\
& \leq C|\ln T|^{1 / q_{0}}\left\|\left(\phi(t)^{2}-|x|^{2}\right)^{1 / q_{0}} F_{T}\right\|_{L^{\frac{q_{0}}{q_{0}^{-1}}}\left(\mathbb{R}_{+}^{1+n}\right)},
\end{aligned}
$$

where $C>0$ depends on $m$ and $n$. Hence, (7.5) holds for supp $F(\cdot, y) \subseteq\{s>0: \phi(s)<$ $\phi(1 / 2) / 8\}$.

\subsubsection{The case $\operatorname{supp} F(\cdot, y) \subseteq\left\{s>0: \phi(s) \geq \frac{1}{8} \phi\left(\frac{1}{2}\right)\right\}$.}

Due to the scaling argument in (4.4), we assume that

$$
\operatorname{supp} F_{T} \subseteq\left\{(s, y): \phi(s)^{2}-|y|^{2} \geq 1 / T^{m+2}, \quad s \geq \phi^{-1}(\phi(1 / 2) / 8)\right\} .
$$

Note that for $(s, y)$ being away from the characteristic cone, the estimate of $w_{T}$ in $(7.5)$ is just a corollary of Lemma 3.4 in [10]. Indeed, from (3.33) of [10] we have

$$
\|w\|_{L^{q_{0}}\left([0,1 / 2] \times \mathbb{R}^{n}\right)} \leq C\|F\|_{L^{\frac{q_{0}}{q_{0}^{-1}}\left(\mathbb{R}_{+}^{1+n}\right)}} .
$$


Since $s \leq t \leq 1 / 2$ if $\phi(s)-|y|>(1 / 4) \phi(1 / 2)$, the weights on both sides are bounded from below and above and we get

$$
\left\|\left(\phi(t)^{2}-|x|^{2}\right)^{1 / q_{0}-v} w\right\|_{L^{q_{0}\left(\left[0, \frac{1}{2}\right] \times \mathbb{R}^{n}\right)}} \leq C\left\|\left(\phi(t)^{2}-|x|^{2}\right)^{1 / q_{0}+v} F\right\|_{L^{\frac{q_{0}}{q_{0}^{-1}}\left(\mathbb{R}_{+}^{1+n}\right)}} .
$$

Thus, we can further assume that

$$
\begin{array}{r}
\operatorname{supp} F_{T} \subseteq\left\{(s, y): \phi(s)^{2}-|y|^{2} \geq \frac{1}{T^{m+2}}, \quad s \geq \phi^{-1}(\phi(1 / 2) / 8),\right. \\
\quad \phi(s)-|y| \leq \phi(1 / 2) / 4\} .
\end{array}
$$

In this case, we make the important observation that $\phi(t)-\phi(s)$ has a positive lower bound (see Lemma A.1 for a proof). This fact will also be used later in the $L^{\infty}$ estimate 7.25). As in the case $\phi(s) \geq \phi(1 / 2) / 8$, we have

$$
w_{T}(t, x)=\int_{0}^{t} \int_{\mathbb{R}^{n}} e^{i[x \cdot \xi+(\phi(t)-\phi(s))|\xi|]} a(t, s, \xi) \hat{F}(s, \xi) \mathrm{d} \xi \mathrm{d} s,
$$

where the amplitude function $a$ satisfies for $\beta \in \mathbb{N}_{0}^{n}$,

$$
\left|\partial_{\xi}^{\beta} a(t, s, \xi)\right| \leq C(1+\phi(t)|\xi|)^{-\frac{m}{2(m+2)}}(1+\phi(s)|\xi|)^{-\frac{m}{2(m+2)}}|\xi|^{-\frac{2}{m+2}-|\beta|} .
$$

Recalling $s \geq \phi^{-1}(\phi(1 / 2) / 8)$, for some positive $\delta \in\left(0, \frac{m}{2(m+2)}\right]$, we can rewrite $w_{T}(t, x)$ as

$$
w_{T}(t, x)=\int_{0}^{t} \int_{\mathbb{R}^{n}} e^{i[x \cdot \xi+(\phi(t)-\phi(s))|\xi|]}(1+\phi(t)|\xi|)^{-\frac{m}{2(m+2)}} \hat{F}(s, \xi) \frac{\mathrm{d} \xi}{|\xi|^{\frac{2}{m+2}+\delta}} \mathrm{d} s .
$$

As in [7], we shall use the complex interpolation method to estimate (7.21). Set

$$
\begin{gathered}
\left(W^{z} F_{T}\right)(t, x)=\left(z-\frac{(m+2) n+2}{2(m+2)}-\delta\right) e^{z^{2}} \int_{0}^{t} \int_{\mathbb{R}^{n}} e^{i[x \cdot \xi+(\phi(t)-\phi(s))|\xi|]} \\
\times(1+\phi(t)|\xi|)^{-\frac{m}{2(m+2)}} \hat{F}(s, \xi) \frac{\mathrm{d} \xi}{|\xi|^{z}} \mathrm{~d} s
\end{gathered}
$$

where $z \in \mathbb{C}$. Then by Stein's complex interpolation theorem, in order to prove (7.22) it suffices to show that

$$
\left\|W^{z} F_{T}\right\|_{L^{\infty}(\{(t, x): 1 / 2 \leq t \leq 1,|x| \leq \phi(t) / 2\})} \leq C\left\|F_{T}\right\|_{L^{1}\left(\mathbb{R}_{+}^{1+n}\right)}
$$

with $\operatorname{Re} z=\frac{(m+2) n+2}{2(m+2)}+\delta$ and

$$
\left\|W^{z} F_{T}\right\|_{L^{2}(\{(t, x): 1 / 2 \leq t \leq 1,|x| \leq \phi(t) / 2\})} \leq C|\ln T|^{1 / 2}\left\|\left(\phi(t)^{2}-|x|^{2}\right)^{1 / 2} F_{T}\right\|_{L^{2}\left(\mathbb{R}_{+}^{1+n}\right)}
$$


with $\operatorname{Re} z=0$, here we have replaced the number $(((m+2) n+2) \delta) / 4$ with $\delta$ in 7.23$)$.

We next show (7.23). Set $z=((m+2) n+2) /(2(m+2))+\delta+i q$. Note that $\phi(t)-\phi(s)$ is bounded from below. Then 7.23 is valid by the stationary phase method. In this case, for the cut-off function $\beta$ in Lemma 3.2 and $\lambda_{j}=2^{j}$ for $j \geq 0$, we get

$$
\begin{aligned}
\left.\left|q e^{-q^{2}} \int_{|\xi| \geq 1} e^{i[x \cdot \xi+(\phi(t)-\phi(s))|\xi|]} \beta\left(\frac{\xi}{\lambda_{j}}\right)(1+\phi(t)|\xi|)^{-\frac{m}{2(m+2)}}\right| \xi\right|^{-\frac{(m+2) n+2}{2(m+2)}-\delta-i q} \mathrm{~d} \xi \mid \\
\leq C_{n} \lambda_{j}^{\frac{n+1}{2}}(\phi(t)-\phi(s))^{-\frac{n-1}{2}}\left(\lambda_{j} \phi(t)\right)^{-\frac{m}{2(m+2)}} \lambda_{j}^{-\frac{(m+2) n+2}{2(m+2)}-\delta} \\
\leq C_{n}(\phi(t)-\phi(s))^{-\frac{n-1}{2}} \phi(t)^{-\frac{m}{2(m+2)}} \lambda_{j}^{-\delta} \\
\leq C \lambda_{j}^{-\delta}
\end{aligned}
$$

where $C>0$ is a constant depending only on $m$ and $n$. On the other hand, for $|\xi|<1$, one has that

$$
\begin{array}{r}
\left.\left|q e^{-q^{2}} \int_{|\xi| \leq 1} e^{i[x \cdot \xi+(\phi(t)-\phi(s))|\xi|]}(1+\phi(t)|\xi|)^{-\frac{m}{2(m+2)}}\right| \xi\right|^{-\frac{(m+2) n+2}{2(m+2)}-\delta-i q} \mathrm{~d} \xi \mid \\
\quad \leq C \int_{0}^{1} r^{-\frac{(m+2) n+2}{2(m+2)}-\delta} r^{n-1} \mathrm{~d} r \leq C
\end{array}
$$

due to $n-1-((m+2) n+2) /(2(m+2))-\delta=n / 2-1-1 /(m+2)-\delta>-1$ for $n \geq 3$ and $n / 2-1 /(m+2)>m /(2(m+2)) \geq \delta>0$. Thus, combining (7.25) and (7.26) yields (7.23).

Next we show (7.24). Set $\phi(s)=\tau+|y|$. Then we have that, for $\operatorname{Re} z=0$

$$
\begin{array}{r}
\left(W^{z} F_{T}\right)(t, x)=C_{m}\left(z-\frac{(m+2) n+2}{2(m+2)}-\delta\right) e^{z^{2}} \int_{\frac{1}{T^{m+2}}}^{\frac{1}{4} \phi\left(\frac{1}{2}\right)} \int_{\mathbb{R}^{n}} \int_{\mathbb{R}^{n}} e^{i[(x-y) \cdot \xi+(\phi(t)-\tau-|y|)|\xi|]} \\
\quad \times F_{T}\left(\phi^{-1}(\tau+|y|), y\right) s^{-\frac{m}{2}} \frac{\mathrm{d} \xi}{|\xi|^{z}} \mathrm{~d} y \mathrm{~d} \tau .
\end{array}
$$

By Hölder's inequality, we arrive at

$$
\begin{aligned}
& \left|\left(W^{z} F_{T}\right)(t, x)\right| \leq C|\ln T|^{1 / 2}\left\{\int_{1 / T^{m+2}}^{\phi(1 / 2) / 4} \mid \tau^{\frac{1}{2}}\left(z-\frac{(m+2) n+2}{2(m+2)}-\delta\right) e^{z^{2}}\right. \\
& \left.\quad \times\left.\int_{\mathbb{R}^{n}} \int_{\mathbb{R}^{n}} e^{i[(x-y) \cdot \xi+(\phi(t)-\tau-|y|)|\xi|]} F_{T}\left(\phi^{-1}(\tau+|y|), y\right) s^{-m / 2} \frac{\mathrm{d} \xi}{|\xi|^{z}} \mathrm{~d} y\right|^{2} \mathrm{~d} \tau\right\}^{\frac{1}{2}} .
\end{aligned}
$$

Note that $\phi^{-1}((1 / 8) \phi(1 / 2)) \leq s \leq 1$. In order to derive $(7.28)$, it suffices to show that the estimate,

$$
\left\|\left(\tilde{W}^{z} f\right)(\phi(t)-\tau, \cdot)\right\|_{L^{2}\left(\left\{x:|x| \leq \frac{\phi(t)}{2}\right\}\right)} \leq C\|f\|_{L^{2}\left(\mathbb{R}^{n}\right)}
$$


with $\operatorname{Re} z=0$ and supp $f \subseteq\{y:|y|<\phi(t)-\tau\}$ holds, for $\tau \leq(1 / 8) \phi(1 / 2)$ and $1 / 2 \leq t \leq 1$ where

$$
\begin{aligned}
\left(\tilde{W}^{z} f\right)(\phi(t)-\tau, x)= & \left(z-\frac{(m+2) n+2}{2(m+2)}-\delta\right) e^{z^{2}} \\
& \times \int_{\mathbb{R}^{n}} \int_{\mathbb{R}^{n}} e^{i[(x-y) \cdot \xi+(\phi(t)-\tau-|y|)|\xi|]} f(y) \frac{\mathrm{d} \xi}{|\xi|^{z}} \mathrm{~d} y .
\end{aligned}
$$

By Lemma A.1, $\phi(t)-\phi(s)$ has a positive lower bound. Consequently, we have $|y|=$ $\phi(s)-\tau<\phi(t)-\tau$. Therefore, once $(7.29)$ is shown, we get $(7.26)$ by setting $f=\tau^{\frac{1}{2}} s^{-\frac{m}{2}} F_{T}$ (see the proof Lemma A.2).

Next we turn to the proof of (7.29) using the classical $L^{2}$ theorem for Fourier integral operators (see, for example, Theorem 25.3.8 in [12]). For this purpose, we have to demonstrate that the phase function $\varphi=(x-y) \cdot \xi+(\phi(t)-\tau-|y|)|\xi|$ of the Fourier integral operator in $(7.30)$ is non-degenerate. Namely, for the point $(x, y, \xi, \tau)(\xi \neq 0)$ satisfying $\nabla_{\xi} \varphi(x, y, \xi, \tau)=0$, one has

$$
\nabla_{y, \xi} \varphi(x, y, \xi, \tau) \neq 0
$$

and

$$
\operatorname{det}\left(\begin{array}{cc}
\varphi_{x y} & \varphi_{x \xi} \\
\varphi_{y \xi} & \varphi_{\xi \xi}
\end{array}\right)(x, y, \xi, \tau) \neq 0 .
$$

Indeed, in order to prove this, it suffices to verify $\operatorname{det} \varphi_{y \xi} \neq 0$ and $-\xi-(y /|y|)|\xi| \neq 0$ for $\nabla_{\xi} \varphi=0$, since $\nabla_{y} \varphi=-\xi-(y /|y|)|\xi|, \varphi_{x y}=0$, and $\varphi_{x \xi}=I_{n}$ hold. Note that $\phi(t)-\tau-|y|=\phi(t)-\phi(s)$ is bounded from below by Lemma A.1. Then $\nabla_{\xi} \varphi=$ $x-y+(\phi(t)-\tau-|y|)(\xi /|\xi|)=0$ is equivalent to $\xi /|\xi|=-(x-y) /(\phi(t)-\tau-|y|)$ (due to $|y|<\phi(t)-\tau$, we can divide by $\phi(t)-\tau-|y|)$. In addition, $\nabla_{y} \varphi=0$ implies $\xi /|\xi|=-y /|y|$. Combining these two facts yields $|x|=\phi(t)-\tau$. However, this is a contradiction since by our assumption, $\tau=\phi(t)-|x| \geq \phi(t) / 2 \geq(1 / 2) \phi(1 / 2)$ and $\tau=\phi(s)-|y| \leq(1 / 2) \phi(1 / 2)$. It means $\nabla_{y} \varphi \neq 0$. On the other hand, $\operatorname{det} \varphi_{y \xi}=\left|-I_{n}-(y /|y|)(\xi /|\xi|)^{T}\right|=0$ holds if and only if $\xi /|\xi|=-y /|y|$ (but $\xi /|\xi|=-y /|y|$ is impossible due to $\nabla_{y} \varphi \neq 0$ for $\nabla_{\xi} \varphi=0$ ). Therefore, the phase function $\varphi$ is non-degenerate, and it follows from the classical $L^{2}$ bounded theorem of Fourier integral operator that (7.29) holds. Hence, (7.28) and further (7.5) are proven. Then Proposition 7.1 is proven.

\subsection{Estimate for small times}

Based on Proposition 7.1, we now start to prove inequality (7.2). We divide the proof into two parts according to the relative scale of the time variable after scaling. More specifically, 
we suppose

$$
F(t, x) \equiv 0 \quad \text { if } \quad|x|>\phi(t)-1 .
$$

Note that $\operatorname{supp} F \subseteq\{(t, x):|x| \leq \phi(t)-1\}$ implies supp $F \subseteq\left\{(t, x):|x|^{2} \leq \phi^{2}(t)-1\right\}$. Set $F=F^{0}+F^{1}$, where

$$
F^{0}=\left\{\begin{array}{cl}
F, & \phi(t) \leq \frac{\phi(1) \phi(T)}{10 \phi(2)} \\
0, & \phi(t)>\frac{\phi(T) \phi(1)}{10 \phi(2)}
\end{array}\right.
$$

Correspondingly, set $w=w^{0}+w^{1}$, where

$$
\left\{\begin{array}{l}
\left(\partial_{t}^{2}-t^{m} \Delta\right) w^{j}=F^{j} \\
w^{j}(0, x)=0, \quad \partial_{t} w^{j}(0, x)=0, \quad j=0,1 .
\end{array}\right.
$$

In this section, we deal with $w^{1}$, that is, we deal with the relative small time. In this case, motivated by the method for treating semilinear wave equations in [7], we shall divide the region $\left\{(t, x):\left.\left|\phi(t)^{2}-\right| x\right|^{2} \leq 1\right\}$ into some pieces (these pieces can be reduced to the case of $\{(t, x):|x| \leq \phi(t) / 2\}$ in (4.3) by a conformal transformation), and apply Proposition 7.1 to derive (7.2). Indeed, we claim that

$$
\begin{aligned}
& \left\|\left(\phi(t)^{2}-|x|^{2}\right)^{1 / q_{0}-v} w^{1}\right\|_{L^{q_{0}(\{(t, x): T / 2 \leq t \leq T\}}} \\
& \leq C \phi(T)^{-v}|\ln T|^{1 / q_{0}}\left\|\left(\phi(t)^{2}-|x|^{2}\right)^{1 / q_{0}+v} F^{1}\right\|_{L^{\frac{q_{0}}{q_{0}^{-1}}}\left(\mathbb{R}_{+}^{1+n}\right)} .
\end{aligned}
$$

Obviously, (7.33) follows from the following localized bounds for $k \in \mathbb{Z}$,

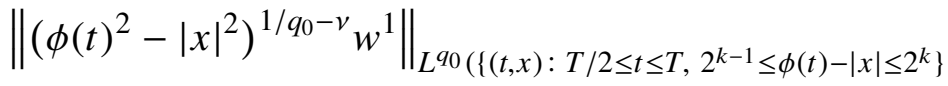

$$
\begin{aligned}
& \leq C|\ln T|^{1 / q_{0}}\left\|\left(\phi(t)^{2}-|x|^{2}\right)^{1 / q_{0}} F^{1}\right\|_{L^{\frac{q_{0}}{q^{-1}}\left(\mathbb{R}_{+}^{1+n}\right)}} .
\end{aligned}
$$

Indeed,

$$
\begin{aligned}
& \left\|\left(\phi(t)^{2}-|x|^{2}\right)^{1 / q_{0}-v} w^{1}\right\|_{L^{q_{0}(\{(t, x): T / 2 \leq t \leq T\})}}
\end{aligned}
$$

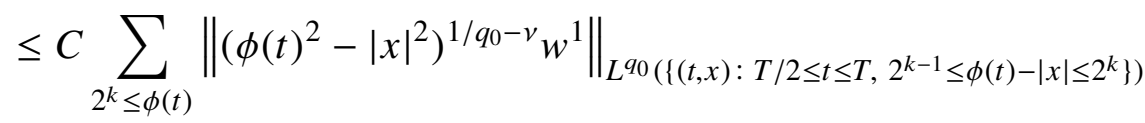

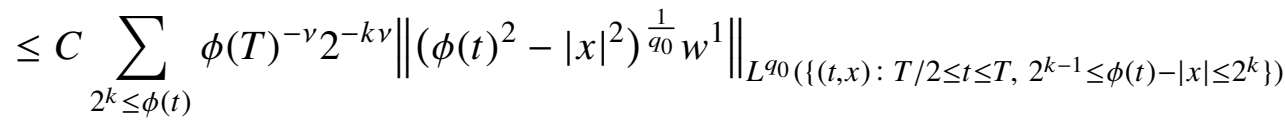

$$
\begin{aligned}
& \leq C \sum_{2^{k} \leq \phi(t)} \phi(T)^{-v} 2^{-k v}|\ln T|^{1 / q_{0}}\left\|\left(\phi(t)^{2}-|x|^{2}\right)^{1 / q_{0}} F^{1}\right\|_{L^{\frac{q_{0}}{q_{0}^{-1}}\left(\mathbb{R}_{+}^{1+n}\right)}} \\
& \leq C \phi(T)^{-v}|\ln T|^{1 / q_{0}}\left\|\left(\phi(t)^{2}-|x|^{2}\right)^{1 / q_{0}} F^{1}\right\|_{L^{\frac{q_{0}}{q_{0}^{-1}}}\left(\mathbb{R}_{+}^{1+n}\right)} \\
& \leq C \phi(T)^{-v}|\ln T|^{\frac{1}{q_{0}}}\left\|\left(\phi(t)^{2}-|x|^{2}\right)^{1 / q_{0}+v} F^{1}\right\|_{L^{\frac{q_{0}}{q_{0}^{-1}}}\left(\mathbb{R}_{+}^{1+n}\right)} \quad\left(\text { by } \phi(t)^{2}-|x|^{2} \geq 1\right)
\end{aligned}
$$


Here we point out that in order that inequality (7.34) makes sense, one needs $2^{k-1} \leq \phi(t)$. Set $T_{k}=T / 2^{\frac{2 k}{m+2}}$ and

$$
w_{k}^{1}=w^{1}\left(2^{\frac{2 k}{m+2}} t, 2^{k} x\right), \quad F_{k}^{1}(t, x)=2^{\frac{4 k}{m+2}} F^{1}\left(2^{\frac{2 k}{m+2}} t, 2^{k} x\right) .
$$

To prove (7.34), it suffices to show that

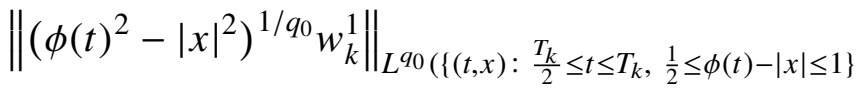

$$
\begin{aligned}
& \leq C(\ln T)^{1 / q_{0}}\left\|\left(\phi(t)^{2}-|x|^{2}\right)^{1 / q_{0}} F^{1}\right\|_{L^{\frac{q_{0}}{q_{0}^{-1}}\left(\mathbb{R}_{+}^{1+n}\right)}} .
\end{aligned}
$$

Now the remaining task is to prove (7.35). At first, we divide the support of $w_{k}$ and $F_{k}$ into suitable pieces. If $T_{k}$ is smaller than some fixed constant, then we choose a $\kappa \in(0,1)$ such that $|x| \leq \kappa \phi(t)$ holds for $T_{k} / 2 \leq t \leq T_{k}$ and $1 / 2 \leq \phi(t)-|x| \leq 1$. Then we can use the same method in Proposition 7.1 to prove the estimate like (7.4). On the other hand, by Lemma A.3 we know that if $T_{k}$ is large, then $|x /| x|-y /| y|| \leq C / \sqrt{\phi\left(T_{k}\right)}$. Thus if we assume that $|y /| y|-v| \leq C / \sqrt{\phi\left(T_{k}\right)}$ holds for some constant vector $v \in \mathbb{R}^{n}$ in the support of $F_{k}$, then $x /|x|$ must be close to $v$ in the support of $w_{k}$. Next we need to prove the estimate

$$
\begin{aligned}
& \left\|\left(\phi(t)^{2}-|x|^{2}\right)^{1 / q_{0}} w_{k}^{1}\right\|_{L^{q_{0}}\left(D_{v, k}\right)} \\
& \quad \leq C|\ln T|^{1 / q_{0}}\left\|\left(\phi(t)^{2}-|x|^{2}\right)^{1 / q_{0}} F_{k}^{1}\right\|_{L^{\frac{q_{0}}{q_{0}-1}}\left(|y /| y|-v| \leq C / \sqrt{\phi\left(T_{k}\right)}, \phi(s) \geq \phi\left(T_{k}\right) / 10\right)},
\end{aligned}
$$

where $D_{v, k}=\left\{(t, x): T_{k} / 2 \leq t \leq T_{k}, 1 / 2 \leq \phi(t)-|x| \leq 1,|x /| x|-v| \leq C / \sqrt{\phi\left(T_{k}\right)}\right\}$. Once 7.36 is done, then we can use Lemma A.4 to sum all the estimates with respect to different constant vectors $v$ to derive (7.35).

To do this, we want to find a transformation which sends $D_{v, k}$ into the center of the characteristic cone and keeps (4.3) invariant Then we use Propostion 7.1 to establish (7.36). However, unlike the case of the wave equation, the Tricomi operator is not the Laplace-Beltrami operator of any metric. Thus it is difficult to find such a transformation. We will fix this gap in a forthcoming paper.

\subsection{Estimate for large times}

Next we estimate $w_{0}$ in (7.32). As in (7.33), it suffices to show that

$$
\begin{aligned}
& \left\|\left(\phi(t)^{2}-|x|^{2}\right)^{1 / q_{0}-v} w^{0}\right\|_{L^{q_{0}(\{(t, x): T / 2 \leq t \leq T\})}} \\
& \leq C \phi(T)^{-v / 4}\left\|\left(\phi^{2}(t)-|x|^{2}\right)^{1 / q_{0}+v} F^{0}\right\|_{L^{\frac{q_{0}}{q_{0}^{-1}}}\left(\mathbb{R}_{+}^{1+n}\right)} .
\end{aligned}
$$


For this purpose, we shall make some reductions. First we suppose supp $F^{0} \subseteq\left[T_{0}, 2 T_{0}\right] \times \mathbb{R}^{n}$ for some fixed constant $T_{0}>0$ satisfying $\phi\left(2 T_{0}\right) \geq 1$. Then (7.37) follows from

$$
\begin{aligned}
\|\left(\phi(t)^{2}-|x|^{2}\right)^{1 / q_{0}-v} w^{0} & \|_{L^{q_{0}}(\{(t, x): T / 2 \leq t \leq T\})} \\
& \leq C \phi\left(T_{0}\right)^{-v / 4} \phi(T)^{-v / 4}\left\|\left(\phi^{2}(t)-|x|^{2}\right)^{1 / q_{0}+v} F^{0}\right\|_{L^{\frac{q_{0}}{q_{0}^{-1}}\left(\mathbb{R}_{+}^{1+n}\right)}}
\end{aligned}
$$

by summing up all over these $T_{0}$. Furthermore, if $F^{0} \equiv 0$ when $\phi(t)-|x|$ is not in $\left[\delta_{0} \phi\left(T_{0}\right), 2 \delta_{0} \phi\left(T_{0}\right)\right]$ for some fixed constant $\delta_{0}$ with $0<\delta_{0} \leq 2$ and $\delta_{0} \phi\left(T_{0}\right) \geq 1$, then in order to prove (7.38) we only need to show

$$
\begin{aligned}
& \left\|\left(\phi(t)^{2}-|x|^{2}\right)^{1 / q_{0}-v} w^{0}\right\|_{L^{q_{0}(\{(t, x): T / 2 \leq t \leq T\})}} \\
& \leq C \phi\left(T_{0}\right)^{-\frac{v}{2}} \phi(T)^{-v / 4}\left\|\left(\phi^{2}(t)-|x|^{2}\right)^{1 / q_{0}+v} F^{0}\right\|_{L^{\frac{q_{0}}{q^{-1}}}\left(\mathbb{R}_{+}^{1+n}\right)} .
\end{aligned}
$$

Finally, if we make a dyadic decomposition with respect to the variable $\phi(t)-|x|$ in the support of $w_{0}$. Then in order to prove (7.39) it suffices to show that, for $\delta \geq \delta_{0}$,

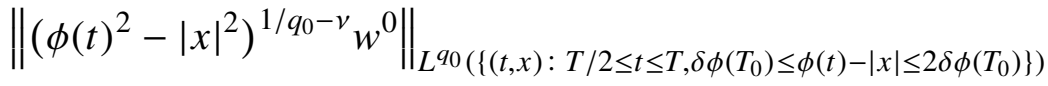

$$
\begin{aligned}
& \leq C \phi\left(T_{0}\right)^{-v / 2} \phi(T)^{-v / 2}\left\|\left(\phi^{2}(t)-|x|^{2}\right)^{1 / q_{0}+v} F^{0}\right\|_{L^{\frac{q_{0}}{q_{0}^{-1}}}\left(\mathbb{R}_{+}^{1+n}\right)} \\
& \leq C\left(\phi\left(T_{0}\right) \phi(T)\right)^{-v / 2}\left\|\left(\phi^{2}(t)-|x|^{2}\right)^{1 / q_{0}+v} F^{0}\right\|_{L^{\frac{q_{0}}{q_{0}^{-1}}}\left(\mathbb{R}_{+}^{1+n}\right)} .
\end{aligned}
$$

With these reductions, to prove (7.40), our task is to establish

$$
\begin{aligned}
\left.\left.\left(\phi\left(T_{0}\right) \phi(T) \delta\right)^{1 / q_{0}-v}\left\|w^{0}\right\|_{L^{q_{0}}(\{(t, x)}: T / 2 \leq t \leq T, \delta \phi\left(T_{0}\right) \leq \phi(t)-|x| \leq 2 \delta \phi\left(T_{0}\right)\right\}\right) & \\
& \leq C\left(\phi\left(T_{0}\right) \phi(T)\right)^{-v / 2}\left(\phi^{2}\left(T_{0}\right) \delta_{0}\right)^{1 / q_{0}+v}\left\|F^{0}\right\|_{L^{\frac{q_{0}}{q_{0}^{-1}}}\left(\mathbb{R}_{+}^{1+n}\right)} .
\end{aligned}
$$

By rearranging some terms, the above inequality follows from

$$
\begin{aligned}
\left(\frac{\phi(T)}{\phi\left(T_{0}\right)}\right)^{1 / q_{0}-v / 2} \delta^{1 / q_{0}+v / 2} \phi\left(T_{0}\right)^{-3 v} \delta^{-3 / 2 v} \delta_{0}^{-v}\left\|w^{0}\right\|_{L^{q_{0}}\left(\left\{(t, x): T / 2 \leq t \leq T, \delta \phi\left(T_{0}\right) \leq \phi(t)-|x| \leq 2 \delta \phi\left(T_{0}\right)\right\}\right)} & \leq C \delta_{0}^{1 / q_{0}}\left\|F^{0}\right\|_{L^{\frac{q_{0}}{q_{0}^{-1}}}\left(\mathbb{R}_{+}^{1+n}\right)} .
\end{aligned}
$$

Note that

$$
\phi\left(T_{0}\right)^{-3 v} \delta^{-3 / 2 v} \delta_{0}^{-v} \leq \phi\left(T_{0}\right)^{-3 v} \delta_{0}^{-5 / 2 v}=\left(\phi\left(T_{0}\right) \delta_{0}\right)^{-3 v} \delta_{0}^{v / 2} \leq \delta_{0}^{v / 2} \leq 2^{v / 2} .
$$

Therefore, (7.40) follows from

$$
\begin{aligned}
\left(\frac{\phi(T)}{\phi\left(T_{0}\right)}\right)^{1 / q_{0}-v / 2} \delta^{1 / q_{0}+v / 2}\left\|w^{0}\right\|_{L^{q_{0}}\left(\left\{(t, x): T / 2 \leq t \leq T, \delta \phi\left(T_{0}\right) \leq \phi(t)-|x| \leq 2 \delta \phi\left(T_{0}\right)\right\}\right)} & \\
& \leq C \delta_{0}^{1 / q_{0}}\left\|F^{0}\right\|_{L^{\frac{q_{0}-1}{q_{0}}}\left(\mathbb{R}_{+}^{1+n}\right)} .
\end{aligned}
$$

We next intend to prove 7.41). Set $G(t, x)=T_{0}^{2} F^{0}\left(T_{0} t, T_{0}^{(m+2) / 2} x\right)$ and $v(t, x)=$ 
$w^{0}\left(T_{0} t, T_{0}^{(m+2) / 2} x\right)$. Then $v$ satisfies

$$
\left\{\begin{array}{l}
\partial_{t}^{2} v-t^{m} \Delta v=G(t, x), \\
v(0, x)=0, \quad \partial_{t} v(0, x)=0,
\end{array}\right.
$$

where $\operatorname{supp} G \subseteq\left\{(t, x): 1 \leq t \leq 2, \delta_{0} \leq \phi(t)-|x| \leq 2 \delta_{0}\right\}$. Then, if we let $T$ denote $T /\left(T_{0}\right)$, then $(7.41)$ is a corollary of

$$
\phi(T)^{1 / q_{0}-v / 2} \delta^{1 / q_{0}+v / 2}\|v\|_{L^{q_{0}}(\{(t, x): T / 2 \leq t \leq T, \delta \leq \phi(t)-|x| \leq 2 \delta\})} \leq C \delta_{0}^{1 / q_{0}}\|G\|_{L^{\frac{q_{0}}{q_{0}-1}}\left(\mathbb{R}_{+}^{1+n}\right)},
$$

where $\phi(T) \geq 10 \phi(2)$ by (7.31).

The proof of $(7.42)$ can be divided into the following three parts according to the different values of $\delta / \delta_{0}$ :

(i) $\delta_{0} \leq \delta \leq 40 \phi(2) /(\phi(1)) \delta_{0}$,

(ii) $\delta \geq 10 \phi(2)$,

(iii) $40 \phi(2) /(\phi(1)) \delta_{0} \leq \delta \leq 10 \phi(2), \delta_{0}<\phi(1) / 4$.

Here we stress the fact that for the wave equation and case (i)-(ii), it is not difficult to prove an analogous inequality (7.42) (see (3.2) and Section 3 of [7]). However, for the Tricomi equation, due to the complexity of its fundamental solution, it needs more delicate and involved techniques from the knowledge of microlocal analysis to get the pointwise estimate. For the proof of (7.42) in case (iii), we shall follow an idea of [7].

\subsubsection{The proof of $(7.42)$ in case (i).}

Note that $\phi(T)>\phi\left(T_{0}\right) \geq 1$ and $\delta \phi\left(T_{0}\right) \leq \phi(T)$. To prove (7.42), it suffices to show

$$
\phi(T)^{1 / q_{0}} v\left\|_{L^{q_{0}}(\{(t, x): T / 2 \leq t \leq T, \delta \leq \phi(t)-|x| \leq 2 \delta\})} \leq C\right\| G \|_{L^{\frac{q_{0}}{q^{-1}}}\left(\mathbb{R}_{+}^{1+n}\right)} .
$$

Using the method of Lemma 3.3 of [10], if we write

$$
v(t, x)=\int_{0}^{t} H(t, s, x) \mathrm{d} s
$$

then we arrive at

$$
\|H(t, s, \cdot)\|_{L^{q_{0}}\left(\mathbb{R}^{n}\right)} \leq C|t-s|^{-\frac{2}{q_{0}}\left(1+\frac{m}{4}\right)}\|G(s, \cdot)\|_{L^{\frac{q_{0}}{q^{-1}}}\left(\mathbb{R}^{n}\right)} .
$$

On the other hand, if we repeat the reduction of (3.23)-(3.24) in [10], we then have $H=\sum_{j=-\infty}^{\infty} H_{j}$, where

$$
H_{j}=T_{j} G(t, s, x)=\int_{\mathbb{R}^{n}} e^{i(x \cdot \xi+(\phi(t)-\phi(s))|\xi|)} \beta\left(|\xi| / 2^{j}\right) a(t, s, \xi) \hat{G}(s, \xi) \mathrm{d} \xi
$$


and the amplitude function $a$ satisfies

$$
\left|\partial_{\xi}^{\beta} a(t, s, \xi)\right| \leq C(1+\phi(t)|\xi|)^{-\frac{m}{2(m+2)}}(1+\phi(s)|\xi|)^{-\frac{m}{2(m+2)}}|\xi|^{-\frac{2}{m+2}-|\beta|} .
$$

If we further set

$$
\bar{T}_{j} G(t, s, x)=\lambda_{j}^{\frac{2 m}{(m+2)((m+2) n+2)}} H_{j}(t, s, x), \quad \lambda_{j}=2^{j},
$$

and repeat the computation of (3.29) and (3.30) in [10], we then have

$$
\begin{aligned}
\left\|\bar{T}_{j} G(t, s, \cdot)\right\|_{L^{2}\left(\mathbb{R}^{n}\right)} \leq \lambda_{j}^{-\frac{2}{m+2}} C\left(1+\lambda_{j} \phi(t)\right)^{-\frac{m}{2(m+2)}} \cdot \frac{(m+2) n-2}{(m+2) n+2} & \\
& \times|t-s|^{-\frac{m}{(m+2) n+2}}\|G(s, \cdot)\|_{L^{2}\left(\mathbb{R}^{n}\right)}
\end{aligned}
$$

and

$$
\begin{aligned}
\left\|\bar{T}_{j} G(t, s, \cdot)\right\|_{L^{\infty}\left(\mathbb{R}^{n}\right) \leq C \lambda_{j}^{\frac{n+1}{2}-\frac{2}{m+2}}} & \left(1+\lambda_{j} \phi(t)\right)^{-\frac{m}{2(m+2)} \cdot \frac{(m+2) n-2}{(m+2) n+2}} \\
& \times|t-s|^{-\frac{m}{(m+2) n+2}}|t-s|^{-\frac{n-1}{2} \cdot \frac{m+2}{2}}\|G(s, \cdot)\|_{L^{1}\left(\mathbb{R}^{n}\right)}
\end{aligned}
$$

Therefore, for $j \geq 0$,

$$
\begin{aligned}
\left\|\bar{T}_{j} G(t, s, \cdot)\right\|_{L^{2}\left(\mathbb{R}^{n}\right)} \leq C \lambda_{j}^{-\frac{2}{m+2}}(1+\phi(t))^{-\frac{m}{2(m+2)} \cdot \frac{(m+2) n-2}{(m+2) n+2}}|t-s|^{-\frac{m}{(m+2) n+2}}\|G(s, \cdot)\|_{L^{2}\left(\mathbb{R}^{n}\right)} \\
\leq C \lambda_{j}^{-\frac{2}{m+2}}(t-s)^{-\frac{m}{4} \cdot \frac{(m+2) n-2}{(m+2) n+2}}|t-s|^{-\frac{m}{(m+2) n+2}}\|G(s, \cdot)\|_{L^{2}\left(\mathbb{R}^{n}\right)}
\end{aligned}
$$

and

$$
\begin{aligned}
\bar{T}_{j} G(t, s, \cdot) & L^{\infty}\left(\mathbb{R}^{n}\right) \\
& \leq C \lambda_{j}^{\frac{n+1}{2}-\frac{2}{m+2}}(t-s)^{-\frac{m}{4} \cdot \frac{(m+2) n-2}{(m+2) n+2}}|t-s|^{-\frac{n-1}{2} \cdot \frac{m+2}{2}}|t-s|^{-\frac{m}{(m+2) n+2}}\|G(s, \cdot)\|_{L^{1}\left(\mathbb{R}^{n}\right)} .
\end{aligned}
$$

Using interpolation, we have that, for $j \geq 0$,

$$
\left\|H_{j}(t, s, \cdot)\right\|_{L^{q}\left(\mathbb{R}^{n}\right)} \leq C(t-s)^{-\left(\frac{m}{4}+1\right) \cdot \frac{(m+2) n-2}{(m+2) n+2}}\|G(s, \cdot)\|_{L^{\frac{q}{q-1}\left(\mathbb{R}^{n}\right)}} .
$$

For $j<0$, let

$$
\tilde{T}_{0} G(t, s, x) \equiv \sum_{j<0} H_{j}(t, s, x)=\int_{|\xi| \leq 1} e^{i(x \cdot \xi+(\phi(t)-\phi(s))|\xi|)} a(t, s, \xi) \hat{G}(s, \xi) \mathrm{d} \xi .
$$

Then it follows from Plancherel's theorem that

$$
\left\|\tilde{T}_{0} G(t, s, \cdot)\right\|_{L^{2}\left(\mathbb{R}^{n}\right)} \leq C\left(\left.\left.\int_{|\xi| \leq 1}|| \xi\right|^{-\frac{2}{m+2}}(1+\phi(t)|\xi|)^{-\frac{m}{2(m+2)}} \hat{G}(s, \xi)\right|^{2} \mathrm{~d} \xi\right)^{1 / 2}
$$


Note that

$$
|\hat{G}(s, \xi)|=\left|\int_{\mathbb{R}^{n}} e^{-i y \cdot \xi} G(s, y) \mathrm{d} y\right|=\left|\int_{|y| \leq \phi(2)} e^{-i y \cdot \xi} G(s, y) \mathrm{d} y\right| \leq C\|G(s, \cdot)\|_{L^{2}\left(\mathbb{R}^{n}\right)}
$$

and

$$
\begin{aligned}
\left(\left.\left.\int_{|\xi| \leq 1}|| \xi\right|^{-\frac{2}{m+2}}(1+\phi(t)|\xi|)^{-\frac{m}{2(m+2)}}\right|^{2} \mathrm{~d} \xi\right)^{1 / 2} & \\
& =C\left(\int_{0}^{1}\left|r^{-\frac{2}{m+2}}(1+\phi(t) r)^{-\frac{m}{2(m+2)}}\right|^{2} r^{n-1} \mathrm{~d} r\right)^{1 / 2} \\
& \leq C\left(\int_{0}^{1} r^{n-1-\frac{m+4}{m+2}} \phi(t)^{-\frac{m}{m+2}} \mathrm{~d} r\right)^{1 / 2} \\
& \leq C \phi(t)^{-\frac{m}{2(m+2)}}
\end{aligned}
$$

Here we have used the fact that $n-1-(m+4) /(m+2) \geq-2 /(m+2)>-1$ for $n \geq 3$ and $m \geq 1$. Thus,

$$
\left\|\tilde{T}_{0} G(t, s, \cdot)\right\|_{L^{2}\left(\mathbb{R}^{n}\right)} \leq C \phi(t)^{-\frac{m}{2(m+2)}}\|G(s, \cdot)\|_{L^{2}\left(\mathbb{R}^{n}\right)} \leq C|t-s|^{-\frac{m}{4}}\|G(s, \cdot)\|_{L^{2}\left(\mathbb{R}^{n}\right)} .
$$

Similarly, we have

$$
\left\|\tilde{T}_{0} G(t, s, \cdot)\right\|_{L^{\infty}\left(\mathbb{R}^{n}\right)} \leq C|t-s|^{-\frac{m}{4}-\frac{n-1}{2} \cdot \frac{m+2}{2}}\|G(s, \cdot)\|_{L^{1}\left(\mathbb{R}^{n}\right)} .
$$

Using interpolation again, we have

$$
\left\|\tilde{T}_{0} G(t, s, \cdot)\right\|_{L^{q_{0}}\left(\mathbb{R}^{n}\right)} \leq C|t-s|^{-\frac{2}{q_{0}}\left(1+\frac{m}{4}\right)}\|G(s, \cdot)\|_{L^{\frac{q_{0}}{q_{0}-1}}\left(\mathbb{R}^{n}\right)} .
$$

Then, by Littlewood-Paley theory, (7.47), (7.51), and (7.44) are proved. Hence, if one notes that $t \lesssim t-s$ holds on the support of $G(s, x)$, then

$$
\begin{aligned}
& \|v\|_{L^{q_{0}}\left([T / 2, T] \times \mathbb{R}^{n}\right)} \leq\left\|\int_{0}^{t}\right\| u(t, s, \cdot)\left\|_{L^{q_{0}\left(\mathbb{R}^{n}\right)}} \mathrm{d} s\right\|_{L_{t}^{q_{0}}} \\
& \leq C\left\|\int_{0}^{t}|t-s|^{-\frac{2}{q_{0}}\left(1+\frac{m}{4}\right)}\right\| G(s, \cdot)\left\|{ }_{L_{x}^{\frac{q_{0}}{q_{0}-1}}} \mathrm{~d} s\right\|_{L_{t}^{q_{0}}} \\
& \leq C\left\||t|^{-\frac{2}{q_{0}}\left(1+\frac{m}{4}\right)} \int_{1}^{2}\right\| G(s, \cdot)\left\|{ }_{L_{x}^{\frac{q_{0}}{q_{0}}-1}} \mathrm{~d} s\right\|_{L_{t}^{q_{0}}} \\
& \leq C\left(\int_{\frac{T}{2}}^{T} t^{\left(-\frac{2}{q_{0}}\left(1+\frac{m}{4}\right)\right) q_{0}} \mathrm{~d} t\right)^{\frac{1}{q_{0}}} \|\left. G\right|_{L^{\frac{q_{0}}{q_{0}^{-1}}}\left(\mathbb{R}_{+}^{n+1}\right)} \quad \text { (Hölder's inequality) } \\
& \leq C T^{-\frac{1}{q_{0}} \frac{m+2}{2}}\|G\|_{L^{\frac{q_{0}}{q_{0}^{-1}}}\left(\mathbb{R}_{+}^{n+1}\right)} \\
& \leq C \phi(T)^{-1 / q_{0}}\|G\|_{L^{\frac{q_{0}}{q_{0}^{-1}}}\left(\mathbb{R}_{+}^{n+1}\right)},
\end{aligned}
$$

which derives (7.43). 


\subsubsection{The proof of $(7.42)$ in case (ii).}

In this case, we have $\phi(t)-|x| \geq \delta \geq 10 \phi(2)$. Similar to the analysis from (7.6) to (7.10), we can write

$$
v=\sum_{j=-\infty}^{\infty} v_{j}=\sum_{j=-\infty}^{\infty} \int_{0}^{t} \int_{\mathbb{R}^{n}} K_{j}(t, x ; s, y) G(s, y) \mathrm{d} y \mathrm{~d} s,
$$

where

$$
K_{j}(t, x ; s, y)=\int_{\mathbb{R}^{n}} e^{i((x-y) \cdot \xi+(\phi(t)-\phi(s))|\xi|)} \beta\left(\frac{|\xi|}{2^{j}}\right) a(t, s, \xi) \hat{G}(s, \xi) \mathrm{d} \xi
$$

Moreover, for $\lambda_{j}=2^{j}$ and $N \in \mathbb{R}^{+}$,

$$
\begin{aligned}
\left|K_{j}(t, x ; s, y)\right| \leq & C \lambda_{j}^{\frac{n+1}{2}-\frac{2}{m+2}}\left(|\phi(t)-\phi(s)|+\lambda_{j}^{-1}\right)^{-\frac{n-1}{2}}\left(1+\phi(t) \lambda_{j}\right)^{-\frac{m}{2(m+2)}} \\
& \times\left(1+\lambda_{j}|| \phi(t)-\phi(s)|-| x-y||\right)^{-N} .
\end{aligned}
$$

Denote $D_{s, y}=\left\{(s, y): 1 \leq s \leq 2, \phi(s)-2 \delta_{0} \leq|y| \leq \phi(s)-\delta_{0}\right\}$. By Hölder's inequality, we arrive at

$$
\left|v_{j}\right| \leq\left\|K_{j}(t, x ; s, y)\left(\phi^{2}(t)-|x|^{2}\right)^{1 / q_{0}}\right\|_{L^{q_{0}\left(D_{s, y}\right)}}\left\|\left(\phi^{2}(t)-|x|^{2}\right)^{-1 / q_{0}} G(s, y)\right\|_{L^{\frac{q_{0}}{q_{0}-1}\left(D_{s, y}\right)}} .
$$

By the assumption on the support of $G$, it is easy to check

$$
\phi(t)-\phi(s)-|x-y| \geq C(\phi(t)-|x|), \quad \phi(t)-\phi(s)+|x-y| \sim \phi(t) .
$$

Based on this, if we set $N=n / 2-1 /(m+2)$ in (7.54), we then have

$$
\begin{aligned}
& \left\|K_{j}(t, x ; s, y)\left(\phi^{2}(t)-|x|^{2}\right)^{1 / q_{0}}\right\|_{L^{q_{0}}\left(D_{s, y}\right)} \\
& \leq C\left(\int _ { 1 } ^ { 2 } \int _ { \phi ( s ) - 2 \delta _ { 0 } \leq | y | \leq \phi ( s ) - \delta _ { 0 } } \left\{\lambda_{j}^{\frac{n+1}{2}-\frac{2}{m+2}}\left(|\phi(t)-\phi(s)|+\lambda_{j}^{-1}\right)^{-\frac{n-1}{2}}\left(1+\phi(t) \lambda_{j}\right)^{-\frac{m}{2(m+2)}}\right.\right. \\
& \left.\left.\quad \times\left(1+\lambda_{j}|| \phi(t)-\phi(s)|-| x-y||\right)^{\frac{1}{m+2}-\frac{n}{2}}\right\}^{q_{0}} \phi(t)(\phi(t)-|x|) \mathrm{d} y \mathrm{~d} s\right)^{1 / q_{0}} \\
& \leq C \phi(t)^{-\frac{n-1}{2}+\frac{1}{q_{0}}-\frac{m}{2(m+2)}}(\phi(t)-|x|)^{\frac{1}{m+2}-\frac{n}{2}+\frac{1}{q_{0}}}\left(\int_{1}^{2} \int_{\phi(s)-2 \delta_{0} \leq|y| \leq \phi(s)-\delta_{0}} \mathrm{~d} y \mathrm{~d} s\right)^{1 / q_{0}} \\
& \leq C \delta_{0}^{\frac{1}{q_{0}}} \phi(t)^{-\frac{n-1}{2}+\frac{1}{q_{0}}-\frac{m}{2(m+2)}}(\phi(t)-|x|)^{\frac{1}{m+2}-\frac{n}{2}+\frac{1}{q_{0}}}
\end{aligned}
$$

and

$$
\left(\int_{1}^{2} \int_{\phi(s)-2 \delta_{0} \leq|y| \leq \phi(s)-\delta_{0}}\left\{\left(\phi(t)^{2}-|x|^{2}\right)^{-1 / q_{0}} G(s, y)\right\}^{\frac{q_{0}}{q_{0}-1}} \mathrm{~d} y \mathrm{~d} s\right)^{\frac{q_{0}-1}{q_{0}}}
$$




$$
\begin{aligned}
& \leq C\left(\delta \phi\left(T_{0}\right) \phi(T)\right)^{-1 / q_{0}}\|G\|_{L^{\frac{q_{0}}{q^{-1}}}\left(\mathbb{R}_{+}^{1+n}\right)} \\
& \leq C(\delta \phi(T))^{-\frac{1}{q_{0}}}\|G\|_{L^{\frac{q_{0}}{q_{0}^{-1}}}\left(\mathbb{R}_{+}^{1+n}\right)} .
\end{aligned}
$$

In addition,

$$
\begin{aligned}
& \left\|\phi(t)^{-\frac{n-1}{2}+\frac{1}{q_{0}}-\frac{m}{2(m+2)}}(\phi(t)-|x|)^{\frac{1}{m+2}-\frac{n}{2}+\frac{1}{q_{0}}}\right\|_{L^{q_{0}}} \\
& \quad=C_{n}\left(\int_{\frac{T}{2}}^{T} \phi(t)^{-\frac{q_{0}}{2}\left(n-\frac{2}{m+2}\right)+1} \int_{0}^{\phi(t)-10 \phi(2)}(\phi(t)-r)^{q_{0}\left(\frac{1}{m+2}-\frac{n}{2}\right)+1} r^{n-1} \mathrm{~d} r \mathrm{~d} t\right)^{1 / q_{0}} \\
& \quad \leq C\left(\int_{\frac{T}{2}}^{T} \phi(t)^{-\frac{2}{m+2}} \mathrm{~d} t\right)^{1 / q_{0}} \\
& \quad \leq C .
\end{aligned}
$$

Therefore, combining (7.55)-(7.57) yields

$$
\begin{aligned}
&\left\|v_{j}\right\|_{L^{q_{0}\left(\mathbb{R}_{+}^{1+n}\right)}} \leq C \delta_{0}^{1 / q_{0}}(\delta \phi(T))^{-1 / q_{0}}\|G\|_{L^{\frac{q_{0}}{q_{0}-1}}\left(\mathbb{R}_{+}^{1+n}\right)} \\
&=C \delta_{0}^{\frac{1}{q_{0}}} \phi(T)^{-\frac{1}{q_{0}}+\frac{v}{2}} \delta^{-\frac{1}{q_{0}}-\frac{v}{2}}\left(\frac{\delta}{\phi(T)}\right)^{\frac{\nu}{2}}\|G\|_{L^{\frac{q_{0}}{q_{0}^{-1}}}\left(\mathbb{R}_{+}^{1+n}\right)} \\
&\left.\leq C \delta_{0}^{\frac{1}{q_{0}}} \phi(T)^{-\frac{1}{q_{0}}+\frac{v}{2}} \delta^{-\frac{1}{q_{0}}-\frac{v}{2}}\|G\|_{L^{\frac{q_{0}}{q_{0}^{-1}}}} \mathbb{R}_{+}^{1+n}\right)
\end{aligned}
$$

here we have used the fact that $\delta \lesssim \phi(T)$ due to $2 \delta \phi\left(T_{0}\right) \leq \phi(T)$ and $\phi\left(T_{0}\right) \geq 1$ before scaling. Together with Lemma 3.2 , this yields the estimate $(7.42)$ in case (ii).

\subsubsection{The proof of $(7.42)$ in case (iii).}

Following the ideas in Section 3 of [7], we shall divide the Fourier integral operator in the expression of $v$ into high frequencies part and low frequencies part. Subsequently, we handle them by different techniques. At first we notice the facts that $t \geq s$ and $1 \leq s \leq 2$ (by the scaling argument in (7.42) $)$. Together with (7.6)-(7.8), this yields that in order to show (7.42), it suffices to estimate, for some $\mu \in\left(0, \frac{m}{2(m+2)}\right]$, the following operator

$$
v=\int_{0}^{t} \int_{\mathbb{R}^{n}} \int_{\mathbb{R}^{n}} e^{i[(x-y) \cdot \xi \pm(\phi(t)-\phi(s))|\xi|]} G(s, y)(1+\phi(t)|\xi|)^{-\frac{m}{2(m+2)}} \frac{\mathrm{d} \xi}{|\xi|^{\frac{2}{m+2}+\mu}} \mathrm{d} y \mathrm{~d} s .
$$

Set $\tau=\phi(s)-|y|$. Applying Hölder's inequality as in (7.35), one then has that

$$
\begin{aligned}
&|v| \leq C \delta_{0}^{\frac{1}{q_{0}}}\left(\int_{\delta_{0}}^{2 \delta_{0}} \mid \int_{\mathbb{R}^{n}} \int_{\mathbb{R}^{n}} e^{i[(x-y) \cdot \xi \pm(\phi(t)-\tau-|y|)|\xi|]} G\left(\phi^{-1}(\tau+|y|), y\right)\right. \\
&\left.\times\left.(1+\phi(t)|\xi|)^{-\frac{m}{2(m+2)}} \frac{\mathrm{d} \xi}{|\xi|^{\frac{2}{m+2}+\mu}} \mathrm{d} y\right|^{\frac{q_{0}}{q_{0}-1}} \mathrm{~d} \tau\right)^{\frac{q_{0}-1}{q_{0}}} .
\end{aligned}
$$


To deal with the integral in (7.58), it is enough to consider the phase function with the minus sign, since for the case of the plus sign the analysis can be done the same way. In addition, we have that: $\delta_{0} \leq(1 / 4) \phi(1)$ and $(1 / 2) \phi(1) \leq|y| \leq \phi(2)$. Indeed, note that $|y| \leq \phi(s) \leq \phi(2)$. One then has $|y| \geq \phi(s)-2 \delta_{0} \geq(1 / 2) \phi(1)$ if $\delta_{0} \leq(1 / 4) \phi(1)$. However, if $\delta_{0}>(1 / 4) \phi(1)$, then $10 \phi(2)<40 \phi(2) /(\phi(1)) \delta_{0} \leq \delta \leq 10 \phi(2)$, which yields a contradiction.

Next we start to estimate $\|v\|_{L_{x}^{q}}$. First note that by (7.31),

$$
\phi(t) \geq \phi\left(\frac{T}{2 T_{0}}\right)=\frac{\phi(T)}{\phi\left(2 T_{0}\right)} \cdot \frac{2}{m+2} \geq 10 \frac{\phi(2)}{\phi(1)} \cdot \phi(1)=10 \phi(2) .
$$

This together with $\tau<\phi(s)<\phi(2)$ yields $\phi(t) \geq \phi(t)-\tau>(1 / 2) \phi(t)$. Thus we can replace $\phi(t)-\tau$ in $(7.58)$ with $\phi(t)$ and consider

$$
\begin{aligned}
&(T g)(t, x)=\int_{\mathbb{R}^{n}} \int_{\left\{y \in \mathbb{R}^{n}: \frac{1}{2} \phi(1) \leq|y| \leq \phi(2)\right\}} e^{i[(x-y) \cdot \xi-(\phi(t)-|y|)|\xi|]} \\
& \times g(y)(1+\phi(t)|\xi|)^{-\frac{m}{2(m+2)}} \frac{\mathrm{d} \xi}{|\xi|^{\frac{2}{m+2}+\mu}} \mathrm{d} y,
\end{aligned}
$$

where $\phi(t) \geq 10 \phi(2)-\phi(2) \geq 9 \phi(2)$ and $\delta<10 \phi(2)$. Then, by Lemma A.5, (7.42) follows from

$$
\|(T g)(t, \cdot)\|_{L^{q_{0}}(\{x: \delta \leq \phi(t)-|x| \leq 2 \delta\})} \leq C \phi(t)^{\frac{v}{2}-\frac{m+4}{q_{0}(m+2)}} \delta^{-\frac{v}{2}-\frac{1}{q_{0}}}\|g\|_{L^{\frac{q_{0}}{q_{0}-1}}\left(\mathbb{R}^{n}\right)} .
$$

Next we focus on the proof of (7.60). As in the proof of Proposition 7.1, we shall use the complex interpolation method to establish (7.60). To do this, set

$$
\begin{aligned}
\left(T_{z} g\right)(t, x)=\left(z-\frac{(m+2) n+2}{2(m+2)}\right) e^{z^{2}} & \int_{\mathbb{R}^{n}} \int_{\mathbb{R}^{n}} \\
& e^{i[(x-y) \cdot \xi-(\phi(t)-|y|)|\xi|]}(1+\phi(t)|\xi|)^{-\frac{m}{2(m+2)}} g(y) \frac{\mathrm{d} \xi}{|\xi|^{z}} \mathrm{~d} y .
\end{aligned}
$$

To prove (7.60), we shall replace $v / 2$ by $v / q_{0}$ and prove

$$
\|(T g)(t, \cdot)\|_{L^{q_{0}}(\{x: \delta \leq \phi(t)-|x| \leq 2 \delta\})} \leq C \phi(t)^{\frac{v}{q_{0}}-\frac{m+4}{q_{0}(m+2)}} \delta^{-\frac{v}{q_{0}}-\frac{1}{q_{0}}}\|g\|_{L^{\frac{q_{0}}{q_{0}-1}}\left(\mathbb{R}^{n}\right)} .
$$

Then for some suitable $\mu>0,(7.60)$ is a consequence of

$$
\left\|\left(T_{z} g\right)(t, \cdot)\right\|_{L^{\infty}\left(\mathbb{R}^{n}\right)} \leq C \phi(t)^{-\frac{n-1}{2}-\frac{m}{2(m+2)}+\mu}\|g\|_{L^{1}\left(\mathbb{R}^{n}\right)}
$$

with $\operatorname{Re} z=\frac{(m+2) n+2}{2(m+2)}+\mu$, and 


$$
\left\|\left(T_{z} g\right)(t, \cdot)\right\|_{L^{2}\left(\mathbb{R}^{n}\right)} \leq C \phi(t)^{-\frac{m}{2(m+2)}}\left(\phi(t)^{\frac{\nu}{2}} \delta^{-\frac{\nu+1}{2}}\right)^{\frac{1}{m+2}}\|g\|_{L^{2}\left(\mathbb{R}^{n}\right)}
$$

with $\operatorname{Re} z=0$. In fact, (7.64) interpolates with (7.63) to give

$$
\|(T g)(t, \cdot)\|_{L^{q_{0}}(\{x: \delta \leq \phi(t)-|x| \leq 2 \delta\})}
$$

$$
\leq C \phi(t)^{\frac{v}{q_{0}(m+2)}+\frac{4 \mu}{(m+2) n+2}-\frac{m+4}{q_{0}(m+2)}} \delta^{-\frac{v+1}{(m+2) q_{0}}}\|g\|_{L^{\frac{q_{0}}{q_{0}-1}\left(\mathbb{R}^{n}\right)}} .
$$

Since $\delta \leq 10 \phi(2)$, we only need $v /\left(q_{0}(m+2)\right)+4 \mu /((m+2) n+2) \leq v / q_{0}$ to derive (7.62). Thus if $0<\mu \leq \min \left(\frac{m}{2(m+2)}, \frac{((m+2) n+2)(m+1)}{4(m+2) q_{0}} v\right)$, then 7.62$)$ is proved.

Compared to (7.24), we have two extra decaying factors $\phi(t)^{-(n-1) / 2-m /(2(m+2))+\mu}$ and $\phi(t)^{v / 2-m /(2(m+2))}$ on the right sides of (7.63) and (7.64) respectively, which require a more involved analysis.

We now prove $(7.63)$ by the stationary phase method. To this end, for the cut-off function $\beta$ in Lemma 3.2. $\lambda_{j}=2^{j}$ with $j \geq 0$ and $z=((m+2) n+2) /(2(m+2))+\mu+i \theta$, we define and estimate the dyadic operator $T_{z}^{j} g$

$$
\begin{aligned}
\left|T_{z}^{j} g\right| & =\left|\theta e^{-\theta^{2}} \int_{\mathbb{R}^{n}} \int_{\mathbb{R}^{n}} e^{i[(x-y) \cdot \xi-(\phi(t)-|y|)|\xi|]} \beta\left(\frac{|\xi|}{\lambda_{j}}\right)(1+\phi(t)|\xi|)^{-\frac{m}{2(m+2)}} g(y) \frac{\mathrm{d} \xi}{|\xi|^{z}} \mathrm{~d} y\right| \\
& \leq C \lambda_{j}^{\frac{n+1}{2}}(\phi(t)-\phi(2))^{-\frac{n-1}{2}}\left(\lambda_{j} \phi(t)\right)^{-\frac{m}{2(m+2)}} \lambda_{j}^{-\frac{(m+2) n+2}{2(m+2)}-\mu}\|g\|_{L^{1}\left(\mathbb{R}^{n}\right)} \\
& \leq C \lambda_{j}^{-\mu} \phi(t)^{-\frac{n-1}{2}-\frac{m}{2(m+2)}}\|g\|_{L^{1}\left(\mathbb{R}^{n}\right)},
\end{aligned}
$$

By summing (7.65) with respect to $j \geq 0$ yields

$$
\left\|\sum_{j=0}^{\infty} T_{z}^{j} g\right\|_{L^{\infty}\left(\mathbb{R}^{n}\right)} \leq C \phi(t)^{-\frac{n-1}{2}-\frac{m}{2(m+2)}}\|g\|_{L^{1}\left(\mathbb{R}^{n}\right)} .
$$

On the other hand, for $|\xi| \leq 1$, we have that

$$
\begin{aligned}
\left|\theta e^{-\theta^{2}} \int_{|\xi| \leq 1} \int_{\mathbb{R}^{n}} e^{i[(x-y) \cdot \xi-(\phi(t)-|y|)|\xi|]}(1+\phi(t)|\xi|)^{-\frac{m}{2(m+2)}} g(y) \frac{\mathrm{d} \xi}{|\xi|} \mathrm{d} y\right| \\
\leq C \int_{|\xi| \leq 1}(1+\phi(t)|\xi|)^{-\frac{n-1}{2}-\frac{m}{2(m+2)}}|\xi|^{-\frac{(m+2) n+2}{2(m+2)}-\mu} \mathrm{d} \xi\|g\|_{L^{1}\left(\mathbb{R}^{n}\right)} \\
\leq C \int_{0}^{1}(1+\phi(t) r)^{-\frac{n-1}{2}-\frac{m}{2(m+2)}} r^{-\frac{n}{2}-\frac{1}{m+2}-\mu} r^{n-1} \mathrm{~d} r\|g\| l_{L^{1}\left(\mathbb{R}^{n}\right)} .
\end{aligned}
$$


Here, we note that $n-1-n / 2-1 /(m+2)=n / 2-1-1 /(m+2)-\mu>-1$ for $0<\mu \leq m /(2(m+2)), m \geq 1$ and $n \geq 3$. Thus, the integral in the last line of (7.67) is convergent. In order to give a precise estimate, set $\rho=(1+\phi(t) r)^{1 /(2(m+2))}$. Then

$$
\begin{aligned}
\int_{0}^{1}(1+ & \phi(t) r)^{-\frac{n-1}{2}-\frac{m}{2(m+2)}} r^{-\frac{n}{2}-\frac{1}{m+2}-\mu} r^{n-1} \mathrm{~d} r \\
& =\int_{1}^{(1+\phi(t))^{\frac{1}{2(m+2)}}} \rho^{2-(m+2) n}\left(\frac{\rho^{2(m+2)}-1}{\phi(t)}\right)^{\frac{n}{2}-1-\frac{1}{m+2}-\mu} \mathrm{d} \frac{\rho^{2(m+2)}-1}{\phi(t)} \\
& \leq C \phi(t)^{-\frac{n}{2}+\frac{1}{m+2}+\mu} \int_{1}^{(1+\phi(t))^{\frac{1}{2(m+2)}}} \frac{\rho^{(m+2) n-2(m+3)-2(m+2) \mu} \rho^{2(m+2)-1}}{\rho^{(m+2) n-2}} \mathrm{~d} \rho \\
& =C \phi(t)^{-\frac{n}{2}+\frac{1}{m+2}+\mu} \int_{1}^{(1+\phi(t))^{\frac{1}{2(m+2)}}} \rho^{-(m+2) \mu-1} \mathrm{~d} \rho \\
& \leq C_{\mu} \phi(t)^{-\frac{n-1}{2}-\frac{m}{2(m+2)}+\mu} .
\end{aligned}
$$

Thus, (7.68) and (7.69) yield (7.63) with a constant $C$ that depends on $m, n$, and $\mu$.

To get (7.64), the small frequencies and large frequencies will be treated separately in the Fourier integral operator of (7.51). As in [7], we shall use the Sobolev trace theorem to handle the small frequencies. More specifically, we first introduce a function $\rho \in C^{\infty}\left(\mathbb{R}^{n}\right)$ such that

$$
\rho(\xi)= \begin{cases}1, & |\xi| \geq 2 \\ 0, & |\xi| \leq 1\end{cases}
$$

For $\alpha=1+v$, we have

$$
\left(T_{z} g\right)(t, x)=\left(R_{z} g\right)(t, x)+\left(S_{z} g\right)(t, x),
$$

where

$$
\begin{aligned}
\left(R_{z} g\right)(t, x)=\left(z-\frac{(m+2) n+2}{2(m+2)}\right) e^{z^{2}} & \int_{\mathbb{R}^{n}} \int_{\frac{1}{2} \phi(1) \leq|y| \leq \phi(2)} e^{i[(x-y) \cdot \xi-(\phi(t)-|y|)|\xi|]} \\
& \times(1+\phi(t)|\xi|)^{-\frac{m}{2(m+2)}}\left(1-\rho\left(\phi(t)^{1-\alpha} \delta^{\alpha} \xi\right)\right) g(y) \frac{\mathrm{d} \xi}{|\xi|^{z}} \mathrm{~d} y, \\
\left(S_{z} g\right)(t, x)=\left(z-\frac{(m+2) n+2}{2(m+2)}\right) e^{z^{2}} & \int_{\mathbb{R}^{n}} \int_{\frac{1}{2} \phi(1) \leq|y| \leq \phi(2)} e^{i[(x-y) \cdot \xi-(\phi(t)-|y|)|\xi|]} \\
& \times(1+\phi(t)|\xi|)^{-\frac{m}{2(m+2)}} \rho\left(\phi(t)^{1-\alpha} \delta^{\alpha} \xi\right) g(y) \frac{\mathrm{d} \xi}{|\xi|^{z}} \mathrm{~d} y .
\end{aligned}
$$

We need to prove that

$$
\left\|\left(R_{z} g\right)(t, \cdot)\right\|_{L^{2}\left(\mathbb{R}^{n}\right)} \leq C \phi(t)^{-\frac{m}{2(m+2)}}\left(\phi(t)^{\alpha-1} \delta^{-\alpha}\right)^{\frac{1}{m+2}}\|g\|_{L^{2}\left(\mathbb{R}^{n}\right)}
$$


for $\operatorname{Re} z=0$, and

$$
\begin{aligned}
\left\|S_{z} g(t, \cdot)\right\|_{L^{2}(\{x: \delta \leq \phi(t)-|x| \leq 2 \delta\})} & \leq C \delta^{-1 / 2}\left(\phi(t)^{\alpha} \delta^{-\alpha}\right)^{-\frac{m}{2(m+2)}}\|g\|_{L^{2}\left(\mathbb{R}^{n}\right)}, \\
& =C \phi(t)^{-\frac{m}{2(m+2)}}\left(\phi(t)^{\alpha-1} \delta^{-\alpha}\right)^{\frac{1}{m+2}}\left(\frac{\delta}{\phi(t)}\right)^{\frac{\alpha-1}{2}}\|g\|_{L^{2}\left(\mathbb{R}^{n}\right)} \\
& \leq C \phi(t)^{-\frac{m}{2(m+2)}}\left(\phi(t)^{\alpha-1} \delta^{-\alpha}\right)^{\frac{1}{m+2}}\|g\|_{L^{2}\left(\mathbb{R}^{n}\right)}
\end{aligned}
$$

for $\operatorname{Re} z=0$. In order to prove (7.70), we shall apply Lemma 3.2 of [7] and a duality argument. For $h \in L^{2}\left(\mathbb{R}^{n}\right)$, due to $\left(R_{z}^{*} h, \bar{g}\right)=\left(h, R_{z}^{-} g\right)$,

$$
\left\|R_{z} g\right\|_{L^{2}}=\sup _{h \in L^{2}} \frac{\left|\left(h, \overline{R_{z}} g\right)\right|}{\|h\|_{L^{2}}} \leq \sup _{h \in L^{2}} \frac{\left\|R_{z}^{*} h\right\|_{L^{2}}}{\|h\|_{L^{2}}}\|g\|_{L^{2}} .
$$

Since

$$
\begin{aligned}
\left(R_{z} g\right)(t, x)=\left(z-\frac{(m+2) n+2}{2(m+2)}\right) e^{z^{2}} & \int_{\mathbb{R}^{n}} \int_{\frac{1}{2} \phi(1) \leq|y| \leq \phi(2)} e^{i[(x-y) \cdot \xi-(\phi(t)-|y|)|\xi|]} \\
& \times\left(1-\rho\left(\phi(t)^{1-\alpha} \delta^{\alpha} \xi\right)\right)(1+\phi(t)|\xi|)^{-\frac{m}{2(m+2)}} g(y) \frac{\mathrm{d} \xi}{|\xi|^{z}} \mathrm{~d} y,
\end{aligned}
$$

the adjoint operator of $R_{z} g$ is

$$
\begin{aligned}
\left(R_{z}^{*} h\right)(y)=\left(\bar{z}-\frac{(m+2) n+2}{2(m+}\right) & 2) e^{\bar{z}^{2}} \iint e^{i[(y-x) \cdot \xi+(\phi(t)-|y|)|\xi|]} \\
& \times\left(1-\rho\left(\phi(t)^{1-\alpha} \delta^{\alpha} \xi\right)\right)(1+\phi(t)|\xi|)^{-\frac{m}{2(m+2)}} h(x) \frac{\mathrm{d} \xi}{|\xi|^{\bar{z}}} \mathrm{~d} x \mathrm{~d} \xi \\
& =\left(\bar{z}-\frac{(m+2) n+2}{2(m+2)}\right) e^{\bar{z}^{2}} \int e^{i(y \cdot \xi-|y||\xi|)} e^{i \phi(t)|\xi|} \\
& \times\left(1-\rho\left(\phi(t)^{1-\alpha} \delta^{\alpha} \xi\right)\right)(1+\phi(t)|\xi|)^{-\frac{m}{2(m+2)}}|\xi|^{-\bar{z}} \hat{h}(\xi) \mathrm{d} \xi
\end{aligned}
$$

Denote

$$
\hat{H}(\xi)=e^{i \phi(t)|\xi|}\left(1-\rho\left(\phi(t)^{1-\alpha} \delta^{\alpha} \xi\right)\right)(1+\phi(t)|\xi|)^{-\frac{m}{2(m+2)}}|\xi|^{-\bar{z}} \hat{h}(\xi)
$$

Then it follows from Lemma 3.2 of [7] and a direct computation that

$\left\|R_{z}^{*} g\right\|_{L^{2}(\phi(1) / 2 \leq|y| \leq \phi(2))}$

$$
\begin{aligned}
& \leq C\left(\|\hat{H}\|_{L^{2}(|\xi| \leq 1)}+\sum_{k=0}^{\infty} 2^{k / 2}\|\hat{H}\|_{L^{2}\left(2^{k} \leq|\xi| \leq 2^{k+1}\right)}\right) \quad \text { (by Lemma } 3.2 \text { of [7]) } \\
& \leq C\left(\|\hat{H}\|_{L^{2}(|\xi| \leq 1)}+\sum_{2^{k+1} \leq \phi(t)^{\alpha-1} \delta^{-\alpha}}^{\infty} 2^{k / 2}\|\hat{H}\|_{L^{2}\left(2^{k} \leq|\xi| \leq 2^{k+1}\right)}\right) .
\end{aligned}
$$

If $k \geq 0$, then

$$
\|\hat{H}\|_{L^{2}\left(2^{k} \leq|\xi| \leq 2^{k+1}\right)} \leq C 2^{-k \frac{m}{2(m+2)}} \phi(t)^{-\frac{m}{2(m+2)}}\|\hat{h}\|_{L^{2}\left(2^{k} \leq|\xi| \leq 2^{k+1}\right)} .
$$


Now the remaining part is the case $|\xi| \leq 1$. We first consider the special case that $\hat{h}(\xi)$ is a polynomial. More specifically, we assume that $\hat{h}(\xi)=\xi_{1}^{l}$. Since the region $\{|\xi| \leq 1\}$ is contained in the region $D=\left\{\left|\xi_{j}\right| \leq 1, j=1, \ldots, n\right\}, D^{\prime}=\left\{\left|\xi_{j}\right| \leq 1, j=2, \ldots, n\right\}$, we only need to estimate the integral

$$
I=\int_{D} \frac{\xi_{1}^{2 l}}{(1+\phi(t)|\xi|)^{\frac{m}{m+2}}} \mathrm{~d} \xi .
$$

But this integral is treated in Lemma A.6, thus (7.70) is established.

Now we turn to deal with (7.71). Denote $K_{z}$ by the kernel of the operator $S_{z}$. Then

$$
\begin{aligned}
K_{z}(t ; x, y)=\left(z-\frac{(m+2) n+2}{2(m+2)}\right) e^{z^{2}} \int_{\mathbb{R}^{n}} e^{i[(x-y) \cdot \xi-(\phi(t)-|y|)|\xi|]} & \\
& \times \rho\left(\phi(t)^{1-\alpha} \delta^{\alpha} \xi\right)(1+\phi(t)|\xi|)^{-\frac{m}{2(m+2)}} \frac{\mathrm{d} \xi}{|\xi|^{z}}
\end{aligned}
$$

with $\operatorname{Re} z=0$. Note that in this case $|\xi| \geq \phi(t)^{\alpha-1} \delta^{-\alpha}$. Therefore, it follows from Lemma 3.3 of [7] and our assumption $\delta \leq 10 \phi(2)$ that, for any $N \in \mathbb{R}^{+}$,

$$
\left|K_{z}\right| \leq C_{N}\left(\frac{\delta}{\phi(t)}\right)^{N}\left(\phi(t)^{\alpha} \delta^{-\alpha}\right)^{-\frac{m}{2(m+2)}} \leq \widetilde{C_{N}}\left(\frac{1}{\phi(t)}\right)^{N}\left(\phi(t)^{\alpha} \delta^{-\alpha}\right)^{-\frac{m}{2(m+2)}}
$$

if ||$x-y|-| \phi(t)-|y||| \geq \delta / 2$.

This yields (7.71) when ||$x-y|-| \phi(t)-|y||| \geq \delta / 2$. For the case of ||$x-y|-| \phi(t)-|y|||<$ $\delta / 2$, analogously treated as in Lemma 3.4-Lemma 3.5 and Proposition 3.6 of [7], (7.71) can also be derived. Here we omit the detail, since the proof is completely similar to that of [7].

Remark 7.3. Note that (7.71) is actually stronger than (7.64) and (7.70) by our assumptions of $\delta \leq \delta \phi\left(T_{0}\right) \leq \phi(T)$ and $T / 2 \leq t \leq T$.

7.70) together with 7.71 yield (7.64). Collecting all the results above, (7.42) is proved.

Based on (7.42), we know that (7.2) is established. 


\section{$8 \quad L^{\mathrm{q}}$ estimate for $\mathrm{q}=\mathbf{2}$}

To prove (6.25), we need the following result, which is a variant of Lemma 3.2 in [7]

Lemma 8.1. One has

$$
\begin{aligned}
& \left\|\int e^{i[x \cdot \xi+t|\xi|]} \hat{f}(\xi) \mathrm{d} \xi\right\|_{L^{2}(\{x: \delta \leq t-|x| \leq 2 \delta\})} \\
& \quad \leq C \delta^{1 / 2}\left(\|\hat{f}\|_{L^{2}(|\xi| \leq 1)}+\sum_{k=0}^{\infty} 2^{k / 2}\|\hat{f}\|_{L^{2}\left(2^{k} \leq|\xi| \leq 2^{k+1}\right)}\right) .
\end{aligned}
$$

Proof. Note that

$$
\begin{aligned}
\left\|\int e^{i[x \cdot \xi+t|\xi|]} \hat{f}(\xi) \mathrm{d} \xi\right\|_{L^{2}(\{x: \delta \leq t-|x| \leq 2 \delta\})} & \\
& =(t-\delta)^{-\frac{n}{2}}\left\|\int e^{i\left[x \cdot \xi+\frac{t}{t-\delta}|\xi|\right]} \hat{f}\left(\frac{\xi}{t-\delta}\right) d \xi\right\|_{L^{2}\left(\left\{x: \frac{t-2 \delta}{t-\delta} \leq|x| \leq 1\right\}\right)} .
\end{aligned}
$$

By applying the Sobolev trace theorem to the function $\int e^{\left.i\left[x \cdot \xi+\frac{t}{t-\delta} \mid \xi\right]\right]} \hat{f}\left(\frac{\xi}{t-\delta}\right) \mathrm{d} \xi$ with respect to the variable $x$, we find that

$$
\begin{aligned}
& \int_{\theta \in S^{n-1}} \mid e^{i\left[r \theta \cdot \xi+\frac{t}{t-\delta}|\xi|\right]}\left.\hat{f}\left(\frac{\xi}{t-\delta}\right) \mathrm{d} \xi\right|^{2} d \theta \\
& \leq C\left(\left\|\hat{f}\left(\frac{\xi}{t-\delta}\right)\right\|_{L^{2}(|\xi| \leq 1)}+\sum_{j=0}^{\infty} 2^{j / 2}\left\|\hat{f}\left(\frac{\xi}{t-\delta}\right)\right\|_{L^{2}\left(2^{j} \leq|\xi| \leq 2^{j+1}\right)}\right)^{2} .
\end{aligned}
$$

Integrating (8.1) with respect to $r$ yields

$$
\begin{aligned}
\left\|\int e^{i[x \cdot \xi+t|\xi|]} \hat{f}(\xi) \mathrm{d} \xi\right\|_{L^{2}(\{x: \delta \leq t-|x| \leq 2 \delta\})} & \leq\left(\frac{t-2 \delta}{t-\delta}\right)^{\frac{n-1}{2}}\left(\frac{\delta}{t-\delta}\right)^{\frac{1}{2}}(t-\delta)^{-\frac{n}{2}}\left(\left\|\hat{f}\left(\frac{\xi}{t-\delta}\right)\right\|_{L^{2}(|\xi| \leq 1)}\right. \\
& \left.+\sum_{j=0}^{\infty} 2^{\frac{j}{2}}\left\|\hat{f}\left(\frac{\xi}{t-\delta}\right)\right\|_{L^{2}\left(2^{j} \leq|\xi| \leq 2^{j+1}\right)}\right) \\
& \leq C \delta^{\frac{1}{2}}\left(\|\hat{f}(\xi)\|_{L^{2}(|\xi| \leq 1)}+\sum_{j=0}^{\infty} 2^{\frac{j}{2}}\|\hat{f}(\xi)\|_{L^{2}\left(2^{j} \leq|\xi| \leq 2^{j+1}\right)}\right) .
\end{aligned}
$$


We now start to prove (4.21). Suppose that $w$ solves 4.3 , where $F \equiv 0$ for $\phi(t)-|x|<1$. By Theorem 2.1 of [36], we have

$$
\|w(t, \cdot)\|_{L^{2}\left(\mathbb{R}^{n}\right)} \leq C t \int_{0}^{t}\|F(s, \cdot)\|_{L^{2}\left(\mathbb{R}^{n}\right)} \mathrm{d} s
$$

which yields, for $0 \leq t \leq 5$,

$$
\|w\|_{L^{2}\left([0,5] \times \mathbb{R}^{n}\right)} \leq C\|F\|_{L^{2}\left([0,5] \times \mathbb{R}^{n}\right)} .
$$

Note, for $0 \leq t \leq 5$, that $\phi(t)-|x|$ is bounded from below and above, thus, for any $v>0$,

$$
\left\|\left(\phi(t)^{2}-|x|^{2}\right)^{\frac{m-2}{2(m+2)}-v} w\right\|_{L^{2}\left([0,5] \times \mathbb{R}^{n}\right)} \leq C\left\|\left(\phi(t)^{2}-|x|^{2}\right)^{\frac{1}{2}+v} F\right\|_{L^{2}\left(\mathbb{R}_{+}^{1+n}\right)}
$$

Next we suppose $\phi(T) \geq 10 \phi(2) / \phi(1)$. We split $w$ as $w=w^{0}+w^{1}$, where

$$
\left\{\begin{array}{l}
\left(\partial_{t}^{2}-t^{m} \Delta\right) w^{j}=F^{j} \\
w^{j}(0, x)=0, \quad \partial_{t} w^{j}(0, x)=0
\end{array}\right.
$$

with

$$
F^{0}=\left\{\begin{array}{cc}
F, & t \leq \frac{T}{10}, \\
0, & t>\frac{T}{10},
\end{array}\right.
$$

and $F=F^{0}+F^{1}$. Then in order to prove 6.25 , it suffices to show that for $j=0,1$,

$$
\begin{aligned}
\left\|\left(\phi(t)^{2}-|x|^{2}\right)^{\frac{m-2}{2(m+2)}-v} w^{j}\right\|_{L^{2}(\{(t, x): T / 2 \leq t \leq T\})} & \\
& \leq C \phi(T)^{-v / 4}\left\|\left(\phi(t)^{2}-|x|^{2}\right)^{1 / 2+v} F^{j}\right\|_{L^{2}\left(\mathbb{R}_{+}^{1+n}\right)}
\end{aligned}
$$

\subsection{Estimate of $w_{1}$}

We first deal with $w^{1}$. As in Proposition 4.2, we shall establish

$$
\left\|\left(\phi(t)^{2}-|x|^{2}\right)^{\frac{m-2}{2(m+2)}} w^{1}\right\|_{L^{2}(\{(t, x): T / 2 \leq t \leq T,|x| \leq \phi(t) / 2\})} \leq C\left\|\left(\phi(t)^{2}-|x|^{2}\right)^{1 / 2} F^{1}\right\|_{L^{2}\left(\mathbb{R}_{+}^{1+n}\right)} .
$$

Set $w_{T}^{1}(t, x)=w^{1}\left(T t, T^{\frac{m+2}{2}} x\right)$ and $F_{T}^{1}(t, x)=T^{2} F^{1}\left(T t, T^{\frac{m+2}{2}} x\right)$. To show (8.4), it suffices to prove that

$$
\left\|\left(\phi(t)^{2}-|x|^{2}\right)^{\frac{m-2}{2(m+2)}} w_{T}^{1}\right\|_{L^{2}\left(\left\{(t, x): 1 / 2 \leq t \leq 1,|x| \leq \frac{\phi(t)}{2}\right\}\right)} \leq C\left\|\left(\phi(t)^{2}-|x|^{2}\right)^{\frac{1}{2}} F_{T}^{1}\right\|_{L^{2}\left(\mathbb{R}_{+}^{1+n}\right)} .
$$


Note that in the domain of integration is $\{(t, x): 1 / 2 \leq t \leq 1,|x| \leq \phi(t) / 2\}$, the behavior of the weight $\left(\phi(t)^{2}-|x|^{2}\right)^{\frac{m-2}{2(m+2)}}$ on the left side of $(8.5)$ behaviors like a positive constant. Then, in order to derive $(8.5)$, we only need to prove

$$
\left\|w_{T}^{1}\right\|_{L^{2}(\{(t, x):|x| \leq \phi(t) / 2,1 / 2 \leq t \leq 1\})} \leq C\left\|\left(\phi(t)^{2}-|x|^{2}\right)^{1 / 2} F_{T}^{1}\right\|_{L^{2}\left(\mathbb{R}_{+}^{n+1}\right)} .
$$

Recall that in $(7.6)$ we have

$$
w_{T}(t, x)=\int_{0}^{t}\left\{V_{2}\left(t, D_{x}\right) V_{1}\left(s, D_{x}\right)-V_{1}\left(t, D_{x}\right) V_{2}\left(s, D_{x}\right)\right\} F_{T}(s, x) \mathrm{d} s .
$$

From 4.7)-(4.40) we know that the amplitude function $a_{1}$ of $V_{2}\left(t, D_{x}\right) V_{1}\left(s, D_{x}\right)$ satisfies

$$
\left|\partial_{\xi}^{\beta} a(t, s, \xi)\right| \leq C t(1+\phi(t)|\xi|)^{-\frac{m+4}{2(m+2)}}(1+\phi(s)|\xi|)^{-\frac{m}{2(m+2)}}|\xi|^{-|\beta|} .
$$

By our assumption, $t \leq 1$. Thus the term on the right side of 8.7 can be controlled by $|\xi|^{-|\beta|}$. The argument for $\left.V_{1}\left(t, D_{x}\right) V_{2}\left(s, D_{x}\right)\right)$ is similar, thus we only need to consider the operators like

$$
\int_{0}^{t} \int_{\mathbb{R}^{n}} e^{i[(x-y) \cdot \xi+(\phi(t)-\phi(s))|\xi|]}|\xi|^{-z} \hat{F}(s, \xi) \mathrm{d} \xi \mathrm{d} s \quad \text { withRe } z=0 .
$$

From this, as in the proof of (7.24), we get 8.6). Furthermore, recall the argument how we use (6.5) to derive (6.1), we use 8.7) and the same method to establish (8.4).

\subsection{Estimation of $\mathbf{w}_{0}$}

Next we estimate $w^{0}$. Note that by an analogous treatment on $w^{0}$ in $(7.38)-(7.42$, , 8.3) will follow from

$$
\phi(T)^{\frac{m-2}{2(m+2)}-\frac{v}{2}} \delta^{\frac{m-2}{2(m+2)}+\frac{v}{2}}\|v\|_{L^{2}(\{(t, x): T / 2 \leq t \leq T, \delta \leq \phi(t)-|x| \leq 2 \delta\})} \leq C \delta_{0}^{1 / 2}\|G\|_{L^{2}\left(\mathbb{R}_{+}^{1+n}\right)},
$$

where $\operatorname{supp} G \subseteq\left\{(t, x): 1 \leq t \leq 2, \delta_{0} \leq \phi(t)-|x| \leq 2 \delta_{0}\right\}, \phi(T) \geq 10 \phi(2) / \phi(1)$, and $\delta \geq \delta_{0}$.

\subsubsection{The case $\delta \geq 10 \phi(2)$}

As in Subsection 7.3.2, we shall use the pointwise estimate to handle the case of $\phi(t)-|x| \geq$ $\delta \geq 10 \phi(2)$. We can write

$$
v=\sum_{j=-\infty}^{\infty} v_{j}=\sum_{j=-\infty}^{\infty} \int_{\mathbb{R}^{n}} \int_{\mathbb{R}^{n}} K_{j}(t, x ; s, y) G(s, y) \mathrm{d} y \mathrm{~d} s,
$$


where

$$
K_{j}(t, x ; s, y)=\int_{\mathbb{R}^{n}} e^{i((x-y) \cdot \xi+(\phi(t)-\phi(s))|\xi|)} \beta\left(|\xi| / 2^{j}\right) a(t, s, \xi) \hat{G}(s, \xi) \mathrm{d} \xi .
$$

By (7.54) and Hölder's inequality, we arrive at

$$
\left|v_{j}\right| \leq\left\|K_{j}(t, x ; s, y)\left(\phi^{2}(t)-|x|^{2}\right)^{1 / 2}\right\|_{L_{s, y}^{2}}\left\|\left(\phi^{2}(t)-|x|^{2}\right)^{-1 / 2} G(s, y)\right\|_{L_{s, y}^{2}} .
$$

Taking $N=n / 2-1 /(m+2)$ in (7.54) and repeating the computations of (7.55) and (7.56), we have

$$
\left\|K_{j}(t, x ; s, y)\left(\phi^{2}(t)-|x|^{2}\right)^{1 / 2}\right\|_{L_{s, y}^{2}} \leq C \delta_{0}^{\frac{1}{2}} \phi(t)^{-\frac{n-1}{2}+\frac{1}{2}-\frac{m}{2(m+2)}}(\phi(t)-|x|)^{\frac{1}{m+2}-\frac{n}{2}+\frac{1}{2}}
$$

and

$$
\left(\int_{\mathbb{R}^{n}} \int_{\mathbb{R}^{n}}\left\{\left(\phi(t)^{2}-|x|^{2}\right)^{-1 / 2} G(s, y)\right\}^{2} \mathrm{~d} y \mathrm{~d} s\right)^{1 / 2} \leq C(\delta \phi(T))^{-1 / 2}\|G\|_{L^{2}\left(\mathbb{R}_{+}^{1+n}\right)} .
$$

In addition, by $-(n-1) / 2+1 /(m+2)<-1 / 2$ for $n \geq 3, m \geq 1$, a direct computation reveals

$$
\begin{aligned}
\left\|\phi(t)^{-\frac{n-1}{2}+\frac{1}{2}-\frac{m}{2(m+2)}}(\phi(t)-|x|)^{-\frac{n-1}{2}+\frac{1}{m+2}}\right\|_{L_{s, y}^{2}} & \\
& \leq C_{n}\left(\int_{\frac{T}{2}}^{T} \phi(t)^{-(n-1)+\frac{2}{m+2}} \int_{0}^{\phi(t)-10 \phi(2)}(\phi(t)-r)^{\frac{2}{m+2}-(n-1)} r^{n-1} \mathrm{~d} r \mathrm{~d} t\right)^{\frac{1}{2}} \\
& \leq C\left(\int_{\frac{T}{2}}^{T} \phi(t)^{\frac{2}{m+2}} \mathrm{~d} t\right)^{\frac{1}{2}} \\
& \leq C T .
\end{aligned}
$$

Thus we obtain

$$
\begin{gathered}
\phi(T)^{\frac{m-2}{2(m+2)}-\frac{v}{2}} \delta^{\frac{m-2}{2(m+2)}+\frac{v}{2}}\|v\|_{L^{2}\left(\left\{(t, x): \frac{T}{2} \leq t \leq T, \delta \leq \phi(t)-|x| \leq 2 \delta\right\}\right)} \\
\quad \leq \phi(T)^{\frac{m-2}{2(m+2)}-\frac{v}{2}} \delta^{\frac{1}{2}+\frac{v}{2}} \delta_{0}^{1 / 2}(\delta \phi(T))^{-\frac{1}{2}} T\|G\|_{L^{2}\left(\mathbb{R}_{+}^{1+n}\right)} \\
\leq C \delta_{0}^{1 / 2}\|G\|_{L^{2}\left(\mathbb{R}_{+}^{1+n}\right)} .
\end{gathered}
$$

\subsubsection{The case $\delta_{0} \leq \delta \leq \mathbf{1 0} \phi(2)$}

Next we study 8.8 in case $\phi(t)-|x| \leq 10 \phi(2)$. At first, we claim that under certain restriction of $\xi$, this case can be treated as the proof of (7.63) in Section 7. Indeed, recalling (4.7)-(4.40) and noting that $t \geq s \geq 1$, we can assume that

$$
v=\int_{0}^{t} \int_{\mathbb{R}^{n}} \int_{\mathbb{R}^{n}} e^{i[(x-y) \cdot \xi+(\phi(t)-\phi(s))|\xi|]} \phi(t)^{-\frac{m}{2(m+2)}}|\xi|^{-1} G(s, y) \mathrm{d} y \mathrm{~d} \xi \mathrm{d} s .
$$


As in Subsection 7.3.2, we split $v$ into a low frequency part and high frequency part. To this end, we choose a function $\beta \in C_{0}^{\infty}\left(\mathbb{R}^{n}\right)$ satisfying $\beta=1$ near the origin such that $v=v_{0}+v_{1}$, where

$$
\begin{aligned}
v_{1} & =\int_{0}^{t} \int_{\mathbb{R}^{n}} \int_{\mathbb{R}^{n}} e^{i[(x-y) \cdot \xi+(\phi(t)-\phi(s))|\xi|]} \phi(t)^{-\frac{m}{2(m+2)}} \frac{1-\beta(\delta \xi)}{|\xi|} G(s, y) \mathrm{d} y \mathrm{~d} \xi \mathrm{d} s \\
& =\int_{0}^{t} T_{1}(t, s, x) \mathrm{d} s .
\end{aligned}
$$

Note $(1-\beta(\delta \xi)) /|\xi|=O(\delta)$. Then the expression of $v_{1}$ is similar to 7.61) with $\operatorname{Re} z=0$ except the extra term $\delta \phi(t)^{-\frac{m}{2(m+2)}}$. Consequently we can apply (7.63) to get

$$
\left\|T_{1}(t, s, \cdot)\right\|_{L^{2}\left(\mathbb{R}^{n}\right)} \leq C \phi(t)^{\frac{\nu}{2}-\frac{m}{2(m+2)}} \delta^{-\frac{\nu}{2}+\frac{1}{2}}\|G(s, \cdot)\|_{L^{2}\left(\mathbb{R}^{n}\right)},
$$

which yields

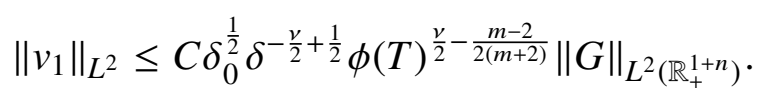

Due to $\delta \leq 10 \phi(2)$, the estimate 8.8 for $v_{1}$ immediately follows.

We now estimate $v_{0}$. At first, one notes that

$$
\begin{aligned}
\mid \int_{|\xi| \leq 1} e^{i[(x-y) \cdot \xi+(\phi(t)-\phi(s))|\xi|]} \phi(t)^{-\frac{m}{2(m+2)} \frac{\beta(\delta \xi)}{|\xi|}} & \mathrm{d} \xi \mid \\
& \leq C(1+|\phi(t)-\phi(s)|)^{-\frac{n-1}{2}} \phi(t)^{-\frac{m}{2(m+2)}} \\
& \leq C(1+|x-y|)^{-\frac{n-1}{2}} \phi(t)^{-\frac{m}{2(m+2)}} .
\end{aligned}
$$

In the last step we have used the fact $\phi(t)-\phi(s) \geq|x-y|$, see LemmaA.1.

Thus it follows from a direct computation that inequality $(8.8)$ holds if we replace $v$ by

$$
v_{01}=\int_{0}^{t} \int_{\mathbb{R}^{n}} \int_{|\xi| \leq 1} e^{i[(x-y) \cdot \xi+(\phi(t)-\phi(s))|\xi|]} \phi(t)^{-\frac{m}{2(m+2)}} \frac{\beta(\delta \xi)}{|\xi|} G(s, y) \mathrm{d} y \mathrm{~d} \xi \mathrm{d} s .
$$

Indeed, one has

$$
\begin{aligned}
& \left\|v_{01}\right\|_{L^{2}\left(\left\{(t, x): \frac{T}{2} \leq t \leq T, \delta \leq \phi(t)-|x| \leq 2 \delta\right\}\right)} \\
& \quad \leq\left\|\iiint_{|\xi| \leq 1} e^{i[(x-y) \cdot \xi+(\phi(t)-\phi(s))|\xi|]} \phi(t)^{-\frac{m}{2(m+2)}} \frac{\beta(\delta \xi)}{|\xi|} \mathrm{d} \xi G(s, y) \mathrm{d} y \mathrm{~d} s\right\|_{L_{t, x}^{2}} \\
& \quad \leq C\left\|\iint(1+|x-y|)^{-\frac{n-1}{2}} \phi(t)^{-\frac{m}{2(m+2)}} G(s, y) \mathrm{d} y \mathrm{~d} s\right\|_{L_{t, x}^{2}} \\
& \quad \leq C \phi(T)^{-\frac{m}{2(m+2)}}\left\|\left(\left\|(1+|x-y|)^{-\frac{n-1}{2}}\right\|_{L_{s, y}^{2}}\|G\|_{L^{2}}\right)\right\|_{L_{t, x}^{2}} \\
& \quad \leq C \phi(T)^{-\frac{m}{2(m+2)}}\|G\|_{L^{2}}\left\|\left(\int_{\frac{T}{2}}^{T} \int_{\delta \leq \phi(t)-|x| \leq 2 \delta}(1+|x-y|)^{-(n-1)} \mathrm{d} x \mathrm{~d} t\right)^{1 / 2}\right\|_{L_{s, y}^{2}} .
\end{aligned}
$$


By $|y| \leq \phi(2),(1 / 2)|x| \leq|x-y| \leq 2|x|$ if $|x| \geq 2 \phi(2)$. On the other hand, if $|x|<2 \phi(2)$, then the integral with respect to $x$ in last line is finite and can be controlled by $\delta$. This yields

$$
\begin{aligned}
\left\|\left(\int_{\frac{T}{2}}^{T} \int_{\delta \leq \phi(t)-|x| \leq 2 \delta}(1+|x-y|)^{-(n-1)} \mathrm{d} x \mathrm{~d} t\right)^{1 / 2}\right\|_{L_{s, y}^{2}} \\
\leq C\left\|\left(\int_{\frac{T}{2}}^{T} \int_{\phi(t)-2 \delta}^{\phi(t)-\delta} \mathrm{d} r \mathrm{~d} t\right)^{1 / 2}\right\|_{L_{s, y}^{2}} \leq C\left(\delta_{0} \delta T\right)^{1 / 2},
\end{aligned}
$$

which implies that the left side of 8.8 is controlled by

$$
\phi(T)^{\frac{m-2}{2(m+2)}-\frac{v}{2}} \delta^{\frac{m-2}{2(m+2)}+\frac{v}{2}} \phi(T)^{-\frac{m}{2(m+2)}}\left(\delta_{0} \delta T\right)^{\frac{1}{2}}\|G\|_{L^{2}} \leq C \delta_{0}^{\frac{1}{2}}\|G\|_{L^{2}\left(\mathbb{R}_{+}^{n+1}\right)} .
$$

Consequently, our proof will be complete if we can show that

$$
\phi(T)^{\frac{m-2}{2(m+2)}-\frac{v}{2}} \delta^{\frac{m-2}{2(m+2)}+\frac{\nu}{2}}\left\|v_{02}\right\|_{L^{2}\left(\left\{(t, x): \frac{T}{2} \leq t \leq T, \delta \leq \phi(t)-|x| \leq 2 \delta\right\}\right)} \leq C \delta_{0}^{\frac{1}{2}}\|G\|_{L^{2}\left(\mathbb{R}_{+}^{n+1}\right)},
$$

where

$$
v_{02}=\int_{0}^{t} \int_{\mathbb{R}^{n}} \int_{|\xi| \geq 1} e^{i[(x-y) \cdot \xi+(\phi(t)-\phi(s))|\xi|]} \phi(t)^{-\frac{m}{2(m+2)}} \frac{\beta(\delta \xi)}{|\xi|} G(s, y) \mathrm{d} y \mathrm{~d} \xi \mathrm{d} s .
$$

The first step in proving 8.12 is to notice that

$$
\left\|v_{02}\right\|_{L^{2}\left(\left\{(t, x): \frac{T}{2} \leq t \leq T, \delta \leq \phi(t)-|x| \leq 2 \delta\right\}\right)} \leq\left\|\int\right\| \tilde{T} G\left\|_{L^{2}(\{x: \delta \leq \phi(t)-|x| \leq 2 \delta\})} \mathrm{d} s\right\|_{L^{2}(\{t: T / 2 \leq t \leq T\})},
$$

where

$$
\tilde{T} G=\iint_{|\xi| \geq 1} e^{i[(x-y) \cdot \xi+(\phi(t)-\phi(s))|\xi|]} \phi(t)^{-\frac{m}{2(m+2)}} \frac{\beta(\delta \xi)}{|\xi|} G(s, y) \mathrm{d} y \mathrm{~d} \xi .
$$

To estimate $\|\tilde{T} G\|_{L^{2}(\{x: \delta \leq \phi(t)-|x| \leq 2 \delta\})}$. It follows from Lemma 8.1 and a direct computation that

$$
\begin{aligned}
& \phi(T)^{\frac{m-2}{2(m+2)}-\frac{v}{2}} \delta^{\frac{m-2}{2(m+2)}+\frac{v}{2}}\left\|v_{02}\right\|_{L^{2}\left(\left\{(t, x): \frac{T}{2} \leq t \leq T, \delta \leq \phi(t)-|x| \leq 2 \delta\right\}\right)} \\
& \leq C \phi(T)^{\frac{m-2}{2(m+2)}-\frac{v}{2}} \delta^{\frac{m-2}{2(m+2)}+\frac{v}{2}} \delta^{\frac{1}{2}} T^{\frac{1}{2}} \phi(T)^{-\frac{m}{2(m+2)}} \\
& \quad \times\left(\sum_{j=0}^{\infty} 2^{\frac{j}{2}}\left\|\iint e^{i[-y \cdot \xi-\phi(s))|\xi|]} \frac{\beta(\delta \xi)}{|\xi|} G(s, y) \mathrm{d} y \mathrm{~d} s\right\|_{L^{2}\left(2^{j} \leq|\xi| \leq 2^{j+1}\right)}\right) \\
& \leq C \delta^{\frac{m}{m+2}}\left(\sum_{j=0}^{\infty}\left\|\iiint_{2^{j} \leq|\xi| \leq 2^{j+1}} e^{i[(x-y) \cdot \xi-\phi(s))|\xi|]} \frac{\beta(\delta \xi)}{|\xi|^{\frac{1}{2}}} G(s, y) \mathrm{d} y \mathrm{~d} \xi \mathrm{d} s\right\|_{L_{x}^{2}}\right) .
\end{aligned}
$$


Then applying Hölder's inequality as in (7.58) yields

$$
\begin{aligned}
& \left\|\iiint_{2^{j} \leq|\xi| \leq 2^{j+1}} e^{i[(x-y) \cdot \xi-\phi(s))|\xi|]} \frac{\beta(\delta \xi)}{|\xi|^{\frac{1}{2}}} G(s, y) \mathrm{d} y \mathrm{~d} \xi \mathrm{d} s\right\|_{L_{x}^{2}} \\
& \leq C \delta_{0}^{1 / 2}\left(\iint_{2^{j} \leq|\xi| \leq 2^{j+1}} e^{i[(x-y) \cdot \xi-(|y|+\tau)|\xi|]} \frac{\beta(\delta \xi)}{|\xi|^{\frac{1}{2}}} G\left(\phi^{-1}(|y|+\tau), y\right) \mathrm{d} y \mathrm{~d} \xi \mid \mathrm{d} x \mathrm{~d} \tau\right)^{1 / 2} .
\end{aligned}
$$

On the other hand, by applying Lemma 3.2 of [7], we obtain that, for each fixed $j \geq 0$,

$$
\begin{aligned}
& \left.\left\|\iint_{2^{j} \leq|\xi| \leq 2^{j+1}} e^{i[(x-y) \cdot \xi-(|y|+\tau)|\xi|]} \frac{\beta(\delta \xi)}{|\xi|^{\frac{1}{2}}} G\left(\phi^{-1}(|y|+\tau), y\right) \mathrm{d} y \mathrm{~d} \xi\right\|_{L_{\tau, x}^{2}}\left|G\left(\phi^{-1}(|y|+\tau), y\right)\right|^{2} \mathrm{~d} y \mathrm{~d} \tau\right)^{\frac{1}{2}} \\
& \quad \leq C\left(\iint_{\phi(1) \leq|y|+\tau \leq \phi(2)}\left|G\left(\phi^{-1}(|y|+\tau), y\right)\right|^{2} \mathrm{~d} \tau \mathrm{d} y\right)^{\frac{1}{2}} \quad \text { (Fubini's Theorem) } \\
& \quad=C\left(\iint_{\phi(1) \leq|y|+\tau \leq \phi(2)}^{2}|G(s, y)|^{2} s^{\frac{m}{2}} \mathrm{~d} s \mathrm{~d} y\right)^{\frac{1}{2}} \\
& \quad \leq C\left(\left.\int_{\mathbb{R}^{n}} \int_{1}^{2}\left|\int_{\mathbb{R}^{n}} \int_{1}^{2}\right| G(s, y)\right|^{2} \mathrm{~d} s \mathrm{~d} y\right)^{\frac{1}{2}} \\
& \quad \leq C\|G\|_{L^{2}\left(\mathbb{R}_{+}^{n+1}\right) \quad \text { (by Fubini's Theorem). }}
\end{aligned}
$$

Furthermore, in the support of $\beta(\delta \xi)$, one has $2^{j} \delta \leq|\xi| \delta \leq C$, which yields

$$
j \leq C(1+|\ln \delta|) .
$$

Substituting (8.15) and (8.16) into (8.14) and further (8.13), we have

$$
\begin{aligned}
\phi(T)^{\frac{m-2}{2(m+2)}-\frac{v}{2}} \delta^{\frac{m-2}{2(m+2)}+\frac{\nu}{2}}\left\|v_{02}\right\|_{L^{2}\left(\left\{(t, x): \frac{T}{2} \leq t \leq T, \delta \leq \phi(t)-|x| \leq 2 \delta\right\}\right)} & \\
& \leq C \delta^{\frac{m}{m+2}}(1+|\ln \delta|) \delta_{0}^{\frac{1}{2}}\|G\|_{L^{2}\left(\mathbb{R}_{+}^{n+1}\right)} \\
& \leq C \delta_{0}^{\frac{1}{2}}\|G\|_{L^{2}\left(\mathbb{R}_{+}^{n+1}\right)} .
\end{aligned}
$$

Hence, we have completed the proof of 6.25. 



\section{A Appendix}

Lemma A.1. Under condition (7.18), $\phi(t)-\phi(s)$ admits a positive lower bound.

Proof. From Theorem 2.4 of [37], we have that

$$
\begin{aligned}
w_{T}(t, x)=C_{m} & \int_{0}^{t} \int_{0}^{\phi(t)-\phi(s)} \partial_{r_{1}} \psi_{\left(F_{T}\right)_{s}}\left(r_{1}, x\right)\left(\phi(t)+\phi(s)+r_{1}\right)^{-\gamma} \\
& \times\left(\phi(t)+\phi(s)-r_{1}\right)^{-\gamma} G(\gamma, \gamma, 1, z) \mathrm{d} r_{1} \mathrm{~d} s
\end{aligned}
$$

where $z=\frac{\left(-r_{1}+\phi(t)-\phi(s)\right)\left(-r_{1}-\phi(t)+\phi(s)\right)}{\left(-r_{1}+\phi(t)+\phi(s)\right)\left(-r_{1}-\phi(t)-\phi(s)\right)}, G(\gamma, \gamma, 1, z)$ is the hypergeometric function, and $\psi_{g}\left(r_{1}, x\right)$ stands for the solution of the linear wave equation

$$
\left\{\begin{array}{l}
\partial_{r_{1}}^{2} v-\Delta v=0 \\
v(0, x)=0, \quad \partial_{r_{1}} v(0, x)=g(x) .
\end{array}\right.
$$

Then by finite propagation speed for the linear wave equation and the expression (A.1), we have that, for any $(s, y) \in \operatorname{supp} F_{T}$ and $(t, x) \in \operatorname{supp} w_{T}$,

$$
\phi(t)-\phi(s) \geq|x-y|
$$

Denote by $w_{T}=\left\langle K(t, x ; s, y), F_{T}(s, y)\right\rangle$. Then, for any fixed $(x, y)$, if $(t, x ; s, y) \in \operatorname{supp} K$, it follows from the support condition of $F_{T}$ that $\phi(t)-\phi(s)$ takes its maximum $|x-y|$ when $\phi(t)-\phi(s)=|x-y|$ and $\phi(s)-|y|=(1 / 4) \phi(1 / 2)$. In fact, since the support of $K$ with respect to the space variable is symmetric, we can assume $x=(|x|, 0, \ldots, 0)$. Figure A.1 illustrates the case when $s$ takes its minimum.

In Figure A.1, $l_{1}$ denotes the surface $\phi(s)-|y|=1 /\left(T^{m+2}\right), l_{2}$ denotes the surface $\phi(s)-|y|=(1 / 4) \phi(1 / 2)$, and $l_{3}$ is the characteristic cone $\phi(\bar{t})-\phi(s)=|\bar{x}-y|$ for fixed $(\bar{t}, \bar{x})$. The domain $A B C D$ is the admissible area for $(s, y)$ when $y_{1}>0$. It is clear that $s$ assumes its maximum at the point $C$, where $\phi(\bar{t})-\phi(s)$ assumes its minimum.

We now show that $\phi(t)-\phi(s)$ has a positive lower bound: Fix $(t, x) \in\{(t, x): 1 / 2 \leq t \leq$ $1,|x| \leq(1 / 2) \phi(t)\}$, (otherwise, rotate in the $x$ variable), we assume $x=(|x|, 0, \ldots, 0)$ and 


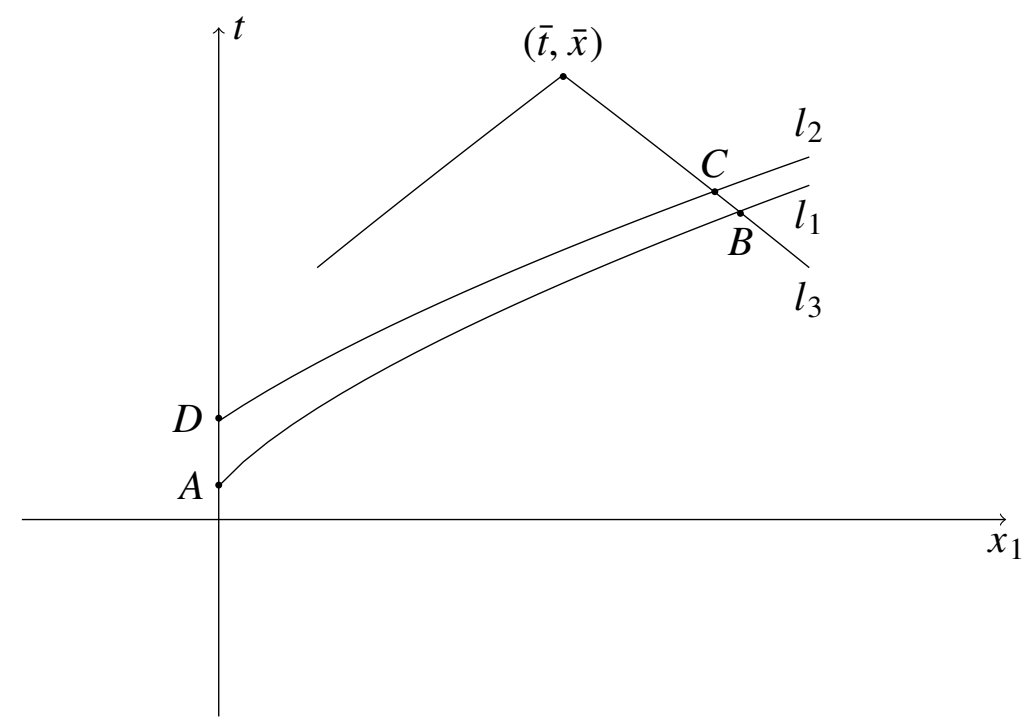

Figure A.1: The boundary of the domain

denote by $y=\left(y_{1}, y^{\prime}\right)$. By $\phi(t)-\phi(s)=|x-y|$ and $\phi(s)-|y|=a$ with $a=(1 / 4) \phi(1 / 2)$, we have

$$
2(\phi(t)-a) \phi(s)=\phi^{2}(t)-a^{2}+2|x| y_{1}-|x|^{2} .
$$

Since $\phi(t)-a \geq \phi(1 / 2)-(1 / 4) \phi(1 / 2)>0, \phi(s)$ is a monotone increasing function of $y_{1}$. So, we may take $y=(|y|, 0)$ to derive a lower bound of $\phi(t)-\phi(s)$. In this case, we have

$$
2(\phi(t)-a) \phi(s)=\phi^{2}(t)-a^{2}+2|x|(\phi(s)-a)-|x|^{2},
$$

which yields, $\phi(s)=(\phi(t)+|x|+a) / 2$ and

$$
\phi(t)-\phi(s)=\frac{\phi(t)-|x|-a}{2} \geq \frac{\phi(t)-\phi(t) / 2-a}{2} \geq \phi(1 / 2) / 8>0 .
$$

Thus the proof of Lemma A. 1 is complete.

Lemma A.2. Setting $f=\tau^{1 / 2} s^{-m / 2} F_{T}$ in (7.29), we get (7.24).

Proof. It follows from (7.28), $f=\tau^{1 / 2} s^{-m / 2} F_{T}$, and the assumption $\phi(s) \geq(1 / 8) \phi(1 / 2)$ that

$$
\begin{aligned}
& \|\left\{\int \mid \tau^{\frac{1}{2}}\left(z-\frac{(m+2) n+2}{2(m+2)}-\delta\right) e^{z^{2}} \int_{\mathbb{R}^{n}} \int_{\mathbb{R}^{n}} e^{i[(x-y) \cdot \xi+(\phi(t)-\tau-|y|)|\xi|]}\right. \\
& \left.\quad \times\left. F_{T}\left(\phi^{-1}(\tau+|y|), y\right) s^{-\frac{m}{2}} \frac{\mathrm{d} \xi}{|\xi|^{z}} \mathrm{~d} y\right|^{2} d \tau\right\}^{1 / 2} \|_{L^{2}\left(\left\{|x| \leq \frac{\phi(t)}{2}, \frac{1}{2} \leq t \leq 1\right\}\right)} \\
& \leq C\left\{\int\left\|\tau^{\frac{1}{2}} s^{-\frac{m}{2}}\left(\phi(s)^{2}-|y|^{2}\right)^{-\frac{1}{2}}\left(\phi(s)^{2}-|y|^{2}\right)^{\frac{1}{2}} F_{T}\left(\phi^{-1}(\tau+|y|), y\right)\right\|_{L^{2}}^{2} d \tau\right\}^{\frac{1}{2}} \\
& \leq C\left\|\left(\phi(s)^{2}-|y|^{2}\right)^{\frac{1}{2}} F_{T}(s, y)\right\|_{L^{2}} .
\end{aligned}
$$

Together with (7.29), this yields (7.24). 
Lemma A.3. Let $w$ solve (4.3) and denote $w$ as $w=\langle K(t, x ; s, y), F(s, y)\rangle$. If $0 \leq$ $\phi(t)-|x| \leq 1, \phi(t) / 10 \leq \phi(s) \leq \phi(t)$, and $\phi(s)-1 \leq|y| \leq \phi(s)$, then

$$
\left|\frac{x}{|x|}-\frac{y}{|y|}\right| \leq \frac{C}{\sqrt{\phi(t)}} \quad \text { if } \quad(t, x, s, y) \in \operatorname{supp} K(t, x ; s, y)
$$

for some constant $C>0$.

Proof. When $t$ is small, the lemma holds naturally. So we can assume that, say, $\phi(t) \geq 20$. Since $\phi(s) \geq \phi(t) / 10 \geq 2$ and $\phi(s)-1 \leq|y|$, we have $2|y| \geq \phi(s)$. Recall that in A.2) we have

$$
K(t, x ; s, y)=0 \quad \text { if } \quad|x-y|>\phi(t)-\phi(s) .
$$

Then using the identity

$$
|x-y|^{2}=(|x|-|y|)^{2}+2(|x||y|-x \cdot y)=(|x|-|y|)^{2}+|x||y||x /| x|-y /| y||^{2},
$$

we have that $|x-y|^{2} \leq(\phi(t)-\phi(s))^{2}$ is equivalent to

$$
\begin{aligned}
|x /| x|-y /| y||^{2} & \leq \frac{(\phi(t)-\phi(s))^{2}-(|x|-|y|)^{2}}{|x||y|} \\
& =\frac{(\phi(t)-|x|-(\phi(s)-|y|)(\phi(t)+|x|-(\phi(s)+|y|))}{|x||y|} \\
& \leq C \frac{(\phi(t)-|x|)(\phi(t)+|x|)}{\phi(t) \phi(s)} .
\end{aligned}
$$

Now we note that $0 \leq \phi(t)-|x| \leq 1$ and $\phi(s) \geq \phi(t) / 10$. Then the result follows immediately.

Lemma A.4. Suppose that $K(x, y)$ is a measurable function on $\mathbb{R}^{m} \times \mathbb{R}^{n}$ and set

$$
T f(x)=\int K(x, y) f(y) \mathrm{d} y .
$$

Suppose further that we can write $\mathbb{R}^{m}$ and $\mathbb{R}^{n}$ as disjoint unions $\mathbb{R}^{m}=\cup_{j \in \mathbb{Z}^{d}} A_{j}$ and $\mathbb{R}^{n}=\bigcup_{k \in \mathbb{Z}^{d}} B_{k}$, where if $x \in A_{j}$, then $K(x, y)=0$ when $y \in B_{k}$ with $|j-k| \geq C$, for some constant $C$. Then, if we let $T_{j k}$ denote the integral operator with kernel $K_{j k}$, where $K_{j k}(x, y)=K(x, y)$ if $(x, y) \in A_{j} \times B_{k}$ and zero otherwise,

$$
\|T\|_{L^{p} \rightarrow L^{q}} \leq C_{d} \cdot \sup _{j, k}\left\|T_{j k}\right\|_{L^{p} \rightarrow L^{q}}
$$

provided that $1 \leq p \leq q \leq \infty$.

Lemma A.5. If (7.60) holds, then (7.42) holds true. 
Proof. If (7.60) holds, then we have

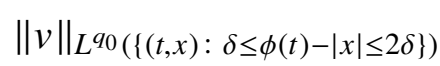

$$
\begin{aligned}
& \leq C \delta_{0}^{\frac{1}{q_{0}}}\left\|\left(\int_{\delta_{0}}^{2 \delta_{0}}\|(T G)(\phi(t)-\tau, \cdot)\|_{L^{q_{0}}(\{x: \delta \leq \phi(t)-|x| \leq 2 \delta\})}^{\frac{q_{0}}{q_{0}}} \mathrm{~d} \tau\right)^{\frac{q_{0}-1}{q_{0}}}\right\|_{L_{t}^{q_{0}}} \\
& \leq C \delta_{0}^{\frac{1}{q_{0}}}\left\|\left(\int\left((\phi(t)-\tau)^{\nu-\frac{m+4}{q_{0}(m+2)}} \delta^{-v-\frac{1}{q_{0}}}\|G(\tau, \cdot)\|_{L^{\frac{q_{0}}{q_{0}-1}}}\right)^{\frac{q_{0}}{q_{0}-1}} \mathrm{~d} \tau\right)^{\frac{q_{0}-1}{q_{0}}}\right\|_{L_{t}^{q_{0}}} \\
& \leq C \delta_{0}^{\frac{1}{q_{0}}}\left\|\phi(t)^{\nu-\frac{m+4}{q_{0}(m+2)}} \delta^{-\nu-\frac{1}{q_{0}}}\left(\int\|G(\tau, \cdot)\|_{L^{\frac{q_{0}}{q_{0}-1}}}^{\frac{q_{0}}{q_{0}-1}} \mathrm{~d} \tau\right)^{\frac{q_{0}-1}{q_{0}}}\right\|_{L_{t}^{q_{0}}} \\
& \leq C \delta_{0}^{\frac{1}{q_{0}}} \delta^{-v-\frac{1}{q_{0}}}\left(\int_{\frac{T}{2}}^{T} \phi(t)^{q_{0} v-\frac{m+4}{m+2}} \mathrm{~d} t\right)^{\frac{1}{q_{0}}}\|G\|_{L^{\frac{q_{0}}{q_{0}-1}}} \\
& \leq C \delta_{0}^{\frac{1}{q_{0}}} \delta^{-v-\frac{1}{q_{0}}} \phi(T)^{\nu-\frac{1}{q_{0}}}\|G\|_{L^{\frac{q_{0}}{q_{0}-1}}},
\end{aligned}
$$

which shows that (7.42) holds.

Lemma A.6. $L^{2}$ estimation in case $|\xi| \leq 1$.

Proof. We first prove the $L^{2}$ estimate for all the polynomials, then use approximation to get the general result. Let $\xi=\left(\xi_{1}, \xi^{\prime}\right)$. For the polynomial function $\xi_{1}^{\alpha}$ we have

$$
\begin{aligned}
I & =\int_{D^{\prime}} \int_{-1}^{1} \frac{\xi_{1}^{2 l}}{(1+\phi(t)|\xi|)^{\frac{m}{m+2}}} \mathrm{~d} \xi_{1} \mathrm{~d} \xi^{\prime} \\
& =\int_{D^{\prime}} \frac{1}{2 l+1} \int_{-1}^{1} \frac{\mathrm{d} \xi_{1}^{2 l+1}}{(1+\phi(t)|\xi|)^{\frac{m}{m+2}}} \mathrm{~d} \xi^{\prime} \\
& =\left.\frac{2}{2 l+1} \int_{D^{\prime}} \frac{1}{(1+\phi(t)|\xi|)^{\frac{m}{m+2}}} \mathrm{~d} \xi^{\prime}\right|_{\xi_{1}=1} \\
& +\frac{m}{(2 l+1)(m+2)} \int_{D^{\prime}} \int_{-1}^{1} \frac{\xi_{1}^{2 l+1} \frac{\xi_{1}}{|\xi|} \phi(t)}{(1+\phi(t)|\xi|)^{\frac{m}{m+2}+1}} \mathrm{~d} \xi_{1} \mathrm{~d} \xi^{\prime} \\
& \leq \frac{2}{2 l+1} \int_{D^{\prime}} \frac{1}{(1+\phi(t))^{\frac{m}{m+2}}} \mathrm{~d} \xi^{\prime}+\frac{m}{(2 l+1)(m+2)} \int_{D^{\prime}} \int_{-1}^{1} \frac{\xi_{1}^{2 l}|\xi| \phi(t)}{(1+\phi(t)|\xi|)^{\frac{m}{m+2}+1}} \mathrm{~d} \xi_{1} \mathrm{~d} \xi^{\prime} \\
& \leq 2 \frac{C_{n}}{2 l+1}(1+\phi(t))^{-\frac{m}{m+2}}+\frac{\xi_{1}^{2 l}}{(2 l+1)(m+2)} \int_{D^{\prime}} \int_{-1}^{1} \frac{\xi_{1}}{(1+\phi(t)|\xi|)^{\frac{m}{m+2}}} \mathrm{~d} \xi_{1} \mathrm{~d} \xi^{\prime},
\end{aligned}
$$

where $C_{n}=\int_{D^{\prime}} \mathrm{d} \xi^{\prime}$ depends only on $n$. Thus we have

$$
\begin{aligned}
& \left(1-\frac{m}{m+2}\right) I \leq 2 \frac{C_{n}}{2 l+1}(1+\phi(t))^{-\frac{m}{m+2}}=2 \int_{D} \xi_{1}^{2 l} \mathrm{~d} \xi(1+\phi(t))^{-\frac{m}{m+2}} \\
& \Longrightarrow\left(\int_{D} \frac{\xi_{1}^{2 l}}{(1+\phi(t)|\xi|)^{\frac{m}{m+2}}} \mathrm{~d} \xi\right)^{\frac{1}{2}} \leq C_{m}\left\|\xi_{1}^{l}\right\|_{L^{2}(D)}(1+\phi(t))^{-\frac{m}{2(m+2)}}
\end{aligned}
$$


Analogously, we can prove similar estimates for all the polynomial functions. Now, for every fixed $t$ and nonzero $h \in L^{2}$, we can find a continuous function $g$, such that

$$
\|\hat{h}-g\|_{L^{2}(D)} \leq \frac{1}{3}\|\hat{h}\|_{L^{2}(D)}(1+\phi(t))^{-\frac{m}{2(m+2)}} .
$$

In addition, since $D$ is compact, by the Stone-Weierstrass theorem, we can find a polynomial $p$ such that

$$
\|p-g\|_{L^{2}(D)} \leq \frac{1}{3}\|\hat{h}\|_{L^{2}(D)}(1+\phi(t))^{-\frac{m}{2(m+2)}} .
$$

Then a approximation argument gives the estimate

$$
\|\hat{H}\|_{L^{2}(D)} \leq C \phi(t)^{-\frac{m}{2(m+2)}}\|\hat{h}\|_{L^{2}(D)} .
$$





\section{Bibliography}

[1] J. Barros-Neto and I. M. Gelfand. Fundamental solutions for the Tricomi operator. I. Duke Math. J. 98 (1999), 465-483.

[2] J. Barros-Neto and I. M. Gelfand. Fundamental solutions for the Tricomi operator. II. Duke Math. J. 111 (2002), 561-584.

[3] J. Barros-Neto and I. M. Gelfand. Fundamental solutions for the Tricomi operator. III. Duke Math. J. 117 (2003), 385-387.

[4] A. Erdelyi. Higher Transcendental Functions. Vol. 1. McGraw-Hill, 1953

[5] A. Erdelyi. Higher Transcendental Functions. Vol. 2. McGraw-Hill, 1953

[6] F. Frankl. On the problems of Chaplygin for mixed sub- and supersonic flows. Bull. Acad. Sci. URSS. Ser. Math. J. 9 (1945), 121-143.

[7] V. Georgiev, H. Lindblad, and C. D. Sogge. Weighted Strichartz estimates and global existence for semi-linear wave equations. Amer. J. Math. 119 (1997), 1291-1319.

[8] R. T. Glassey. Existence in the large for $\square u=F(u)$ in two space dimensions. Math. Z. 178 (1981), 233-261.

[9] R. T. Glassey. Finite-time blow-up for solutions of nonlinear wave equations. Math. Z. 177 (1981), 323-340.

[10] D.-Y. He, I. Witt, and H.-C. Yin. "On the global solution problem of semilinear generalized Tricomi equations, I'. arXiv:1511.08722, 2015

[11] J.-X. Hong and G.-Q. Li. $L^{p}$ estimates for a class of integral operators. J. Partial Differ. Equ. 9 (1996), 343-364.

[12] L. Hörmander. The Analysis of Linear Partial Differertial Equations. III. Grundlehren Math. Wiss. Berlin: Springer, 1985

[13] F. John. Blow-up of solutions of nonlinear wave equations in three space dimensions. Manuscripta Math. 28 (1979), 235-265. 
[14] C.-E. Kenig, G. Ponce, and L. Vega. Well-posedness and scattering results for the generalized Korteweg-de Vries equation via the contraction principle. Comm. Pure Appl. Math. 46 (1993), 527-620.

[15] H. Lindblad and C. D. Sogge. On existence and scattering with minimal regularity for semilinear wave equations. J. Funct. Anal 130 (1995), 357-426.

[16] H. Lindblad and C. D. Sogge. Long time existence for small amplitude semilinear wave equations. Amer. J. Math. 118 (1996), 1047-1135.

[17] D. Lupo, D. D. Monticelli, and K. R. Payne. Variational characterizations of weak solutions to the Dirichlet problem for mixed-type equations. Comm. Pure Appl. Math. 68 (2015), 1569-1586.

[18] D. Lupo, C. S. Morawetz, and K. R. Payne. On closed boundary value problems for equations of mixed elliptic-hyperbolic type. Comm. Pure Appl. Math. 60 (2007), $1319-1348$.

[19] D. Lupo and K. R. Payne. Critical exponents for semilinear equations of mixed elliptic-hyperbolic and degenerate types. Comm. Pure Appl. Math. 56 (2003), 403424.

[20] D. Lupo and K. R. Payne. Conservation laws for equations of mixed elliptichyperbolic and degenerate types. Duke Math. J. 127 (2005), 251-290.

[21] M. Christ. Lectures on Singular Integral Operators. Vol. 77. CBMS Regional Conf. Ser. in Math., 1990

[22] M. Christ and K. Kiselev. A Maximal Functions Associated to Filtrations. J. Funct. Anal. 179 (2001), 409-425.

[23] M. Christ and M. Weinstein. Dispersion of low-amplitude solutions of generalized Korteweg-de Vries equation. J. Funct. Anal. 259 (1991), 87-109.

[24] M. A. Rammaha. Finite-time blow-up for nonlinear wave equations in high dimensions. Comm. Partial Differential Equations 12 (1987), 677-700.

[25] Z.-P. Ruan, I. Witt, and H.-C. Yin. The existence and singularity structures of low regularity solutions to higher order degenerate hyperbolic equations. J. Differential Equations 256 (2014), 407-460.

[26] Z.-P. Ruan, I. Witt, and H.-C. Yin. On the existence and cusp singularity of solutions to semilinear generalized Tricomi equations with discontinuous initial data. Commun. Contemp. Math. 17 (2015), 1450028, 49 pp. 
[27] Z.-P. Ruan, I. Witt, and H.-C. Yin. On the existence of low regularity solutions to semilinear generalized Tricomi equations in mixed type domains. J. Differential Equations 259 (2015), 7406-7462.

[28] Z.-P. Ruan, I. Witt, and H.-C. Yin. "On the existence of solutions with minimal regularity for semilinear generalized Tricomi equations, Preprint”. arXiv:1608.01826. 2016

[29] J. Schaeffer. The equation $\square u=|u|^{p}$ for the critical value of p. Proc. Royal Soc. Edinburgh Sect. 101 (1985), 31-44.

[30] T. Sideris. Nonexistence of global solutions to semilinear wave equations in high dimensions. J. Differential Equations 52 (1984), 378-406.

[31] C. D. Sogge. Fourier Integrals in Classical Analysis. Vol. 105. Cambridge Tracts in Math. Cambridge: Cambridge Univ. Press, 1993

[32] W. Strauss. Nonlinear scattering theory at low energy. J. Funct. Anal 41 (1981), $110-133$.

[33] R. Strichartz. A priori estimates for the wave equation and some applications. $J$. Funct. Anal 5 (1970), 218-235.

[34] F. Tricomi. Sulle equazioni lineari alle derivate parziali di secondo ordine, di tipo misto. Atti Accad. Naz. Lincei Mem. Cl. Fis. Mat. Nat. 5 (1923), 134-247.

[35] K. Yagdjian. A note on the fundamental solution for the Tricomi-type equation in the hyperbolic domain. J. Differential Equations 206 (2004), 227-252.

[36] K. Yagdjian. Global existence for the $n$-dimensional semilinear Tricomi-type equations. Comm. Partial Diff. Equations 31 (2006), 907-944.

[37] K. Yagdjian. Integral transform approach to generalized Tricomi equations. $J$. Differential Equations 11 (2015), 5927-5981.

[38] B. Yordanov and Q. S. Zhang. Cauchy problem for semilinear wave equations in four space dimensions with small initial data. J. Differential Equations 8 (1995), 135-144.

[39] B. Yordanov and Q. S. Zhang. Finite time blow up for critical wave equations in high dimensions. J. Funct. Anal. 231 (2006), 361-374. 